\title{
Metabolites with Antioxidant Activity from Marine Macroalgae
}

\author{
Leto-Aikaterini Tziveleka $^{1} \mathbb{D}$, Mohamed A. Tammam ${ }^{1,2} \mathbb{D}$, Olga Tzakou $^{1}$, Vassilios Roussis ${ }^{1}$ (D) \\ and Efstathia Ioannou $1, *$ (D)
}

1 Section of Pharmacognosy and Chemistry of Natural Products, Department of Pharmacy, National and Kapodistrian University of Athens, Panepistimiopolis Zografou, 15771 Athens, Greece; ltziveleka@pharm.uoa.gr (L.-A.T.); mtammam@pharm.uoa.gr (M.A.T.); tzakou@pharm.uoa.gr (O.T.); roussis@pharm.uoa.gr (V.R.)

2 Department of Biochemistry, Faculty of Agriculture, Fayoum University, Fayoum 63514, Egypt

* Correspondence: eioannou@pharm.uoa.gr; Tel.: +30-210-727-4913

Citation: Tziveleka, L.-A.; Tammam,

M.A.; Tzakou, O.; Roussis, V.;

Ioannou, E. Metabolites with

Antioxidant Activity from Marine

Macroalgae. Antioxidants 2021, 10,

1431. https://doi.org/10.3390/

antiox10091431

Academic Editor: Luca Sebastiani

Received: 3 August 2021

Accepted: 3 September 2021

Published: 8 September 2021

Publisher's Note: MDPI stays neutral with regard to jurisdictional claims in published maps and institutional affiliations.

Copyright: (c) 2021 by the authors. Licensee MDPI, Basel, Switzerland. This article is an open access article distributed under the terms and conditions of the Creative Commons Attribution (CC BY) license (https:/ / creativecommons.org/licenses/by/ $4.0 /)$.

\begin{abstract}
Reactive oxygen species (ROS) attack biological molecules, such as lipids, proteins, enzymes, DNA, and RNA, causing cellular and tissue damage. Hence, the disturbance of cellular antioxidant homeostasis can lead to oxidative stress and the onset of a plethora of diseases. Macroalgae, growing in stressful conditions under intense exposure to UV radiation, have developed protective mechanisms and have been recognized as an important source of secondary metabolites and macromolecules with antioxidant activity. In parallel, the fact that many algae can be cultivated in coastal areas ensures the provision of sufficient quantities of fine chemicals and biopolymers for commercial utilization, rendering them a viable source of antioxidants. This review focuses on the progress made concerning the discovery of antioxidant compounds derived from marine macroalgae, covering the literature up to December 2020. The present report presents the antioxidant potential and biogenetic origin of 301 macroalgal metabolites, categorized according to their chemical classes, highlighting the mechanisms of antioxidative action when known.
\end{abstract}

Keywords: macroalgae; marine metabolites; antioxidant activity; scavenging; reactive oxygen species

\section{Introduction}

In all aerobic organisms, oxygen is a crucial element in their metabolic pathways. A high redox potential milieu stimulates the production of free radicals, defined as chemical species with unpaired valence electrons [1]. The most common reactive species in biological systems are oxygen radicals or oxygen-derived species, such as superoxide anion $\left(\mathrm{O}_{2}{ }^{-}\right)$, hydrogen peroxide $\left(\mathrm{H}_{2} \mathrm{O}_{2}\right)$, and hydroxyl radicals $(\cdot \mathrm{OH})[2,3]$, collectively named reactive oxygen species (ROS). Still, other forms of radicals, such as nitric oxide (NO·) and transition metal ions, can also be produced. ROS are generated as products of normal cellular functioning and oxygen metabolism and have essential functions in various important biochemical processes, such as the defense against infections, vasodilation, neurotransmission, gene regulation, and oxidative signaling [3,4].

Disturbance of the equilibria of prooxidant/antioxidant reactions in cells can lead to redox imbalance and oxidative stress, which causes an excessive generation of ROS and free radicals, in turn resulting in severe cellular damage (Figure 1) [3,5-9]. These molecules further react with key organic substrates, such as DNA, RNA, proteins, and lipids, leading to disruption of their structure or function, and consequently to the onset of diseases, such as atherosclerosis [10], diabetes [11], rheumatoid arthritis [12], inflammatory diseases [13], neurodegenerative diseases [14,15], aging, immune system disorders, and cancer [16,17].

The defense system of living organisms against free radicals comprises both enzymatic and non-enzymatic antioxidants [18]. Enzymes either prevent the formation of or neutralize free radicals (e.g., superoxide dismutases (SOD), catalases (CAT), lactoperoxidases, and glutathione peroxidases (GPx)), or indirectly neutralize free radicals by supporting the 
activity of other endogenous antioxidants (e.g., glutathione reductase (GR) and glucose6-phosphate dehydrogenase) [19]. On the other hand, non-enzymatic antioxidants are compounds, other than enzymes, that act on free radicals and can be either produced by the stressed living organism or delivered through the diet, e.g., via the consumption of ascorbic acid (vitamin C), tocopherol (vitamin E), $\beta$-carotene, flavonoids, and polyphenols [20]. The most effective and extensively used strategy to diminish oxidative stress is the supplementation of exogenous antioxidants [21]. In recent years, safety and health concerns have been raised for synthetic antioxidants. Therefore, natural antioxidants have attracted attention and are being widely used [1]. Since 2007, antioxidants have been defined as "any substance that delays, prevents or removes oxidative damage to a target molecule" [2].

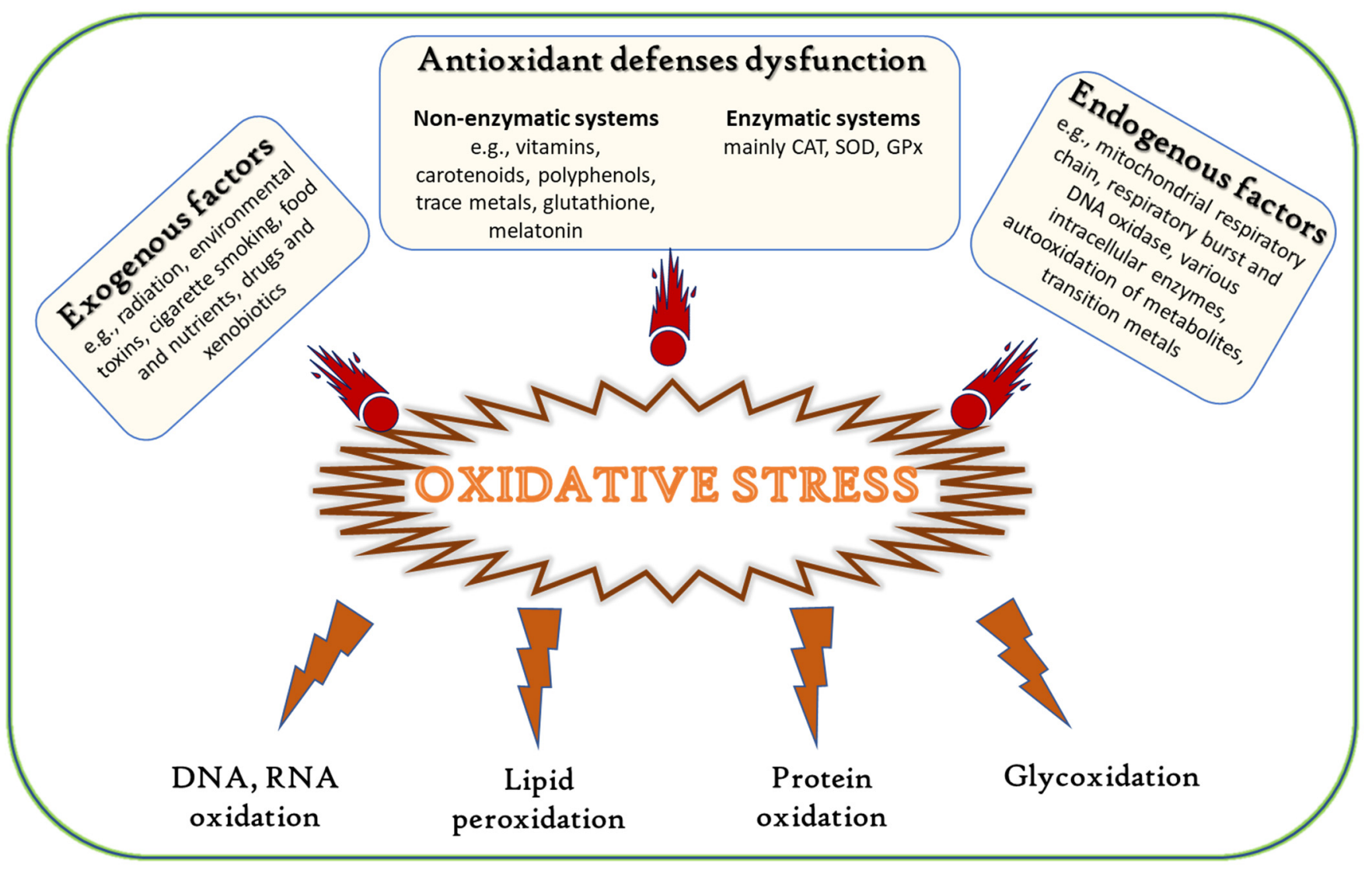

Figure 1. Causes and effects of oxidative stress (adapted from [6]).

Oceans, covering about 70\% of Earth's surface and hosting an immense array of macro- and microorganisms, constitute a renewable resource of potential therapeutic agents. The diverse and antagonistic marine environment triggers the production of a wide variety of bioactive compounds. Marine organisms have adapted remarkably to extreme environmental conditions, such as high salinity, low or high temperature, high pressure, low availability of nutrients, and low or high exposure to sunlight [22], and can, therefore, provide an outstanding reservoir of bioactive compounds, many of which are unprecedented in terrestrial organisms [23-27].

Marine algae constitute a rich source of structurally diverse natural products, often exhibiting significant biological activities [28,29]. Algae are growing in ecosystems with intense exposure to sunlight and high concentrations of oxygen, conditions that favor the production of free radicals. However, the absence of oxidative damage in structural fatty acid membranes suggests that these organisms synthesize compounds with antioxidant activity [30]. In recent years, several studies highlight the antioxidant potential of seaweeds, attributed to natural products belonging to different structural classes [31-36]. 
A high number of compounds isolated from green, brown, and red algae (Chlorophyta, Ochrophyta, and Rhodophyta, respectively) have been proven to exert prominent antioxidant activity. This review compiles the progress made concerning the discovery of antioxidant compounds derived from marine macroalgae, covering the literature up to December 2020. Following a brief overview of the most commonly used methods for the evaluation of antioxidant activity, algal metabolites with antioxidant activity are presented according to their chemical classification in five main groups, namely (1) phenolic compounds, including bromophenols, phlorotannins, and flavonoids, (2) terpenoids, including steroids \& carotenoids, (3) meroterpenoids, (4) nitrogenous compounds, including peptides, alkaloids and chlorophyll-related pigments, and (5) carbohydrates and polysaccharides. Their structural characteristics, the assays used to evaluate their activity, and the measured antioxidant activity levels (when reported in numerical form) are presented, while the mechanisms of antioxidative action are discussed when known.

\section{Brief Overview of the Methods Employed for the Evaluation of Antioxidant Activity}

Efficient antioxidants typically have high redox potential that allows them to act as reducing agents, hydrogen donors, or singlet oxygen quenchers. There are many techniques for evaluating the antioxidant activity, including free radical scavenging, oxygen scavenging, singlet oxygen quenching, metal chelation and inhibition of oxidative enzymes [37]. Overall, in vitro antioxidant tests using free radical traps are relatively straightforward to perform. However, antioxidant activity cannot be securely proposed based on the results from a single assay due to the differences observed between the various test systems [38]. Huang et al. (2005) roughly classified the most important antioxidant capacity assays, according to the reactions involved, into two types: (a) the hydrogen atom transfer (HAT)based reactions which quantify hydrogen atom donating capacity, and (b) the electron transfer (ET)-based reactions which measure the reducing capacity of antioxidants [39] (Table 1). In HAT-based assays, the antioxidant and the substrate compete for peroxyl radicals. The most commonly used HAT-based assays include the oxygen radical absorbance capacity (ORAC) [40] and the total radical trapping antioxidant potential (TRAP) [41] assays. On the other hand, in ET-based assays the capacity of an antioxidant to reduce an oxidant is measured. The most common ET-based assays include the determination of the total phenolics content (TPC) using the Folin-Ciocalteu reagent [42], the trolox equivalence antioxidant capacity (TEAC)/2,2-azino-bis(3-ethyl benzothiazoline-6-sulfonic acid) diammonium salt $\left(\mathrm{ABTS}^{+}\right)$radical scavenging [43], the ferric reducing antioxidant power (FRAP) [44,45], and 1,1-diphenyl-2-picrylhydrazyl (DPPH) [46,47] assays.

Antioxidant activity evaluation can also be performed in vivo in animal models, such as in Wistar rats or mice. SOD, CAT, glutathione (GSH), GPx, oxidized low-density lipoprotein (LDL), malondialdehyde (MDA), and GR are the major in vivo indicators of oxidative stress that are usually monitored $[48,49]$.

Table 1. A list of the most commonly used in vitro assays for the determination of antioxidant activity (adapted from [50]).

2,2-azino-bis (3-ethyl benzothiazoline-6-sulfonic acid) diammonium salt $\left(\mathrm{ABTS}^{+}\right.$) radical scavenging [51]

$\beta$-carotene bleaching [52]

crocin bleaching [53]

Hydrogen atom

transfer

(HAT)-based

assays hydrogen peroxide $\left(\mathrm{H}_{2} \mathrm{O}_{2}\right)$ scavenging [54]

hydroxyl radical averting capacity (HORAC) [55] hydroxyl scavenging [56]

inhibited oxygen uptake (IOU) [57]

lipid peroxidation inhibition capacity (LPIC) [58]

oxygen radical absorbance capacity (ORAC) [40]

photochemiluminescence (PCL) [59] 
Table 1. Cont.

\begin{tabular}{|c|c|}
\hline $\begin{array}{l}\text { Electron transfer } \\
\text { (ET)-based assays }\end{array}$ & $\begin{array}{c}\text { 1,1-diphenyl-2-picrylhydrazyl (DPPH) free radical scavenging [46,47] } \\
\text { cupric reducing antioxidant capacity (CUPRAC) [60] } \\
\text { ferric reducing antioxidant power (FRAP) }[44,45] \\
\text { ferric thiocyanate (FTC) [61] } \\
\text { nitric oxide radical scavenging [62] } \\
\text { N,N-dimethyl-p-phenylene diamine (DMPD) radical scavenging [63] } \\
\text { peroxyl radical scavenging [64] } \\
\text { potassium ferricyanide reducing power (PFRAP) [65] } \\
\text { superoxide anion radical scavenging [66] } \\
\text { thiobarbituric acid reactive substances (TBARS) [67] } \\
\text { total phenolics content (TPC) using Folin-Ciocalteu reagent [42] } \\
\text { trolox equivalence antioxidant capacity (TEAC) using ABTS [43] }\end{array}$ \\
\hline $\begin{array}{l}\text { Other in vitro } \\
\text { methods }\end{array}$ & $\begin{array}{c}\text { ascorbic acid content [68] } \\
\text { cellular antioxidant activity (CAA) [69] } \\
\text { metal chelating activity [70] } \\
\text { scavenging of phosphomolybdenum [71] } \\
\text { scavenging of xanthine oxidase [72] }\end{array}$ \\
\hline
\end{tabular}

\section{Phenolic Compounds}

Phenols comprise a class of chemical compounds containing an aromatic ring bearing a hydroxyl group. Phenolic compounds are classified either as simple phenols or polyphenols based on the number of phenol units in their molecule. Bromophenols (BPs) are marine secondary metabolites containing one or several phenols with one or more bromine atoms in their molecule. Many BPs have been isolated and identified from a variety of marine species, including red, brown, and green algae, as well as ascidians and sponges [73]. Phlorotannins constitute another important and diverse group of naturally occurring polyphenolic secondary metabolites, restricted though to marine algae. Table 2 presents the phenolic compounds, including BPs, phlorotannins, and flavonoids (Figures 2-8), isolated so far from marine macroalgae that exhibit significant antioxidant activities.

Table 2. Phenolic compounds from macroalgae with antioxidant activity.

\begin{tabular}{|c|c|c|c|}
\hline Compound & Isolation Source & Assay/Activity & Reference \\
\hline 1 & $\begin{array}{c}\text { Symphyocladia latiuscula } \\
\text { (Rhodophyta, Florideophyceae, Ceramiales) }\end{array}$ & DPPH scavenging: $\mathrm{IC}_{50}=14.0 \mu \mathrm{M}$ & {$[74]$} \\
\hline 2 & $\begin{array}{c}\text { Gloiopeltis furcata } \\
\text { (Rhodophyta, Florideophyceae, Gigartinales) }\end{array}$ & $\begin{array}{c}\text { DPPH scavenging: } \mathrm{IC}_{50}=86.2 \mu \mathrm{M} \\
\text { ONOO }^{-} \text {scavenging: } 4.58 \pm 0.01 \mu \mathrm{M}\end{array}$ & {$[75]$} \\
\hline 3 & $\begin{array}{c}\text { Rhodomela confervoides } \\
\text { (Rhodophyta, Florideophyceae, Ceramiales) }\end{array}$ & $\begin{array}{l}\text { ABTS }^{+} \text {scavenging: } \mathrm{IC}_{50}=1.60 \pm 0.04 \mu \mathrm{M} \\
\text { DPPH scavenging: } \mathrm{IC}_{50}=50.6 \pm 0.2 \mu \mathrm{M}\end{array}$ & {$[76]$} \\
\hline 4 & $\begin{array}{c}\text { R. confervoides } \\
\text { (Rhodophyta, Florideophyceae, Ceramiales) }\end{array}$ & $\begin{array}{c}\text { ABTS }{ }^{+} \text {scavenging: } \mathrm{IC}_{50}=1.56 \pm 0.02 \mu \mathrm{M} \\
\text { DPPH scavenging: } \mathrm{IC}_{50}=42.3 \pm 0.2 \mu \mathrm{M} ; 67 \%\end{array}$ & {$[76,77]$} \\
\hline 5 & $\begin{array}{c}\text { S. latiuscula } \\
\text { (Rhodophyta, Florideophyceae, Ceramiales) }\end{array}$ & $\begin{array}{l}\text { bleomycin-dependent DNA damage } \\
\text { deoxyribose assay }\end{array}$ & [78] \\
\hline 6 & $\begin{array}{c}\text { R. confervoides } \\
\text { (Rhodophyta, Florideophyceae, Ceramiales) }\end{array}$ & $\begin{array}{c}\text { ABTS }^{+} \text {scavenging: } \mathrm{IC}_{50}=1.62 \pm 0.03 \mu \mathrm{M} \\
\text { DPPH scavenging: } \mathrm{IC}_{50}=40.5 \pm 0.2 \mu \mathrm{M} ; 30 \%\end{array}$ & {$[76,77]$} \\
\hline 7 & $\begin{array}{c}\text { S. latiuscula } \\
\text { (Rhodophyta, Florideophyceae, Ceramiales) }\end{array}$ & DPPH scavenging: $\mathrm{IC}_{50}=15.5 \mu \mathrm{M}$ & {$[74]$} \\
\hline 8 & $\begin{array}{c}\text { S. latiuscula } \\
\text { (Rhodophyta, Florideophyceae, Ceramiales) }\end{array}$ & DPPH scavenging: $\mathrm{IC}_{50}=7.5 \mu \mathrm{M}$ & [79] \\
\hline
\end{tabular}


Table 2. Cont.

\begin{tabular}{|c|c|c|c|}
\hline Compound & Isolation Source & Assay/Activity & Reference \\
\hline 9 & $\begin{array}{c}\text { R. confervoides } \\
\text { (Rhodophyta, Florideophyceae, Ceramiales) }\end{array}$ & $\begin{array}{l}\text { ABTS }{ }^{+} \text {scavenging: } \mathrm{IC}_{50}=1.36 \pm 0.01 \mu \mathrm{M} \\
\text { DPPH scavenging: } \mathrm{IC}_{50}=38.4 \pm 0.2 \mu \mathrm{M}\end{array}$ & [76] \\
\hline 10 & $\begin{array}{c}\text { R. confervoides } \\
\text { (Rhodophyta, Florideophyceae, Ceramiales) }\end{array}$ & $\begin{array}{l}\text { ABTS }{ }^{+} \text {scavenging: } \mathrm{IC}_{50}=2.11 \pm 0.04 \mu \mathrm{M} \\
\text { DPPH scavenging: } \mathrm{IC}_{50}=7.43 \pm 0.10 \mu \mathrm{M}\end{array}$ & [76] \\
\hline 11 & $\begin{array}{c}\text { R. confervoides } \\
\text { (Rhodophyta, Florideophyceae, Ceramiales) }\end{array}$ & $\begin{array}{l}\text { ABTS }{ }^{+} \text {scavenging: } \mathrm{IC}_{50}=1.87 \pm 0.02 \mu \mathrm{M} \\
\text { DPPH scavenging: } \mathrm{IC}_{50}=20.5 \pm 0.1 \mu \mathrm{M}\end{array}$ & [76] \\
\hline 12 & $\begin{array}{c}\text { S. latiuscula } \\
\text { (Rhodophyta, Florideophyceae, Ceramiales) }\end{array}$ & $\begin{array}{c}\text { ABTS }^{+} \text {scavenging: } \mathrm{IC}_{50}=71.0 \mu \mathrm{M} \\
\text { DPPH scavenging: } \mathrm{IC}_{50}=14.4 ; 18.5 \mu \mathrm{M} \\
\text { CUPRAC } \\
\mathrm{Fe}^{2+} \text { chelation: } \mathrm{IC}_{50}=44.7 \mu \mathrm{M} \\
\text { FRAP } \\
\text { AChE inhibition: } \mathrm{IC}_{50}=13.85 \mathrm{nM} \\
\text { BChE inhibition: } \mathrm{IC}_{50}=38.22 \mathrm{nM}\end{array}$ & {$[74,80]$} \\
\hline 13 & $\begin{array}{c}\text { R. confervoides } \\
\text { (Rhodophyta, Florideophyceae, Ceramiales) }\end{array}$ & $\begin{array}{c}\text { ABTS }^{+} \text {scavenging: } \mathrm{IC}_{50}=8.07 \mu \mathrm{M} ; \\
\text { TEAC }=2.68 \mathrm{mM} \\
\text { DPPH scavenging: } \mathrm{IC}_{50}=12.4 ; 15.9 \mu \mathrm{M} \\
\text { CUPRAC } \\
\mathrm{Fe}^{2+} \text { chelation: } \mathrm{IC}_{50}=65.2 \mu \mathrm{M} \\
\text { FRAP } \\
\text { AChE inhibition: } \mathrm{IC}_{50}=17.10 \mathrm{nM} \\
\text { BChE inhibition: } \mathrm{IC}_{50}=40.57 \mathrm{nM}\end{array}$ & {$[80,81]$} \\
\hline 14 & $\begin{array}{c}\text { R. confervoides } \\
\text { (Rhodophyta, Florideophyceae, Ceramiales) }\end{array}$ & $\begin{array}{c}\text { ABTS }^{+} \text {scavenging: } \mathrm{IC}_{50}=8.1 \mu \mathrm{M} ; \\
\text { TEAC }=2.21 \mathrm{mM} \\
\text { DPPH scavenging: } \mathrm{IC}_{50}=14.6 ; 18.5 \mu \mathrm{M} \\
\text { CUPRAC } \\
\mathrm{Fe}^{2+} \text { chelation: } \mathrm{IC}_{50}=54.6 \mu \mathrm{M} \\
\text { FRAP } \\
\text { AChE inhibition: } \mathrm{IC}_{50}=29.88 \mathrm{nM} \\
\text { BChE inhibition: } \mathrm{IC}_{50}=46.51 \mathrm{nM}\end{array}$ & {$[80,81]$} \\
\hline 15 & $\begin{array}{c}\text { R. confervoides } \\
\text { (Rhodophyta, Florideophyceae, Ceramiales) }\end{array}$ & $\begin{array}{l}\text { ABTS }^{+} \text {scavenging: } \mathrm{TEAC}=2.31 \mathrm{mM} \\
\text { DPPH scavenging: } \mathrm{IC}_{50}=5.43 \mu \mathrm{M}\end{array}$ & [81] \\
\hline 16 & $\begin{array}{c}\text { R. confervoides } \\
\text { (Rhodophyta, Florideophyceae, Ceramiales) }\end{array}$ & $\begin{array}{l}\text { ABTS }^{+} \text {scavenging: } \mathrm{TEAC}=2.14 \mathrm{mM} \\
\text { DPPH scavenging: } \mathrm{IC}_{50}=5.70 \mu \mathrm{M}\end{array}$ & [81] \\
\hline 17 & $\begin{array}{c}\text { S. latiuscula } \\
\text { (Rhodophyta, Florideophyceae, Ceramiales) }\end{array}$ & DPPH scavenging: $\mathrm{IC}_{50}=27.9 \mu \mathrm{M}$ & [82] \\
\hline 18 & $\begin{array}{c}\text { R. confervoides } \\
\text { (Rhodophyta, Florideophyceae, Ceramiales) }\end{array}$ & $\begin{array}{l}\text { ABTS }{ }^{+} \text {scavenging: } \mathrm{TEAC}=4.37 \pm 0.24 \mathrm{mM} \\
\text { DPPH scavenging: } \mathrm{IC}_{50}=3.82 \pm 0.01 \mu \mathrm{M}\end{array}$ & [83] \\
\hline 19 & $\begin{array}{c}\text { R. confervoides } \\
\text { (Rhodophyta, Florideophyceae, Ceramiales) }\end{array}$ & $\begin{array}{l}\text { ABTS }^{+} \text {scavenging: } \mathrm{TEAC}=2.06 \pm 0.08 \mathrm{mM} \\
\text { DPPH scavenging: } \mathrm{IC}_{50}=9.52 \pm 0.04 \mu \mathrm{M}\end{array}$ & [76] \\
\hline 20 & $\begin{array}{c}\text { S. latiuscula } \\
\text { (Rhodophyta, Florideophyceae, Ceramiales) }\end{array}$ & DPPH scavenging: $\mathrm{IC}_{50}=24.0 \mu \mathrm{M}$ & [74] \\
\hline 21 & $\begin{array}{c}\text { Polysiphonia morrowii, Polysiphonia urceolata, } R . \\
\text { confervoides } \\
\text { (Rhodophyta, Florideophyceae, Ceramiales) }\end{array}$ & $\begin{array}{l}\text { DPPH scavenging: } \mathrm{IC}_{50}=20.3 \mu \mathrm{M} \\
\text { cytoprotective effect against cellular oxidative } \\
\text { stress } \\
\text { HO-1 activity and expression in keratinocytes } \\
\text { Nrf2 expression } \\
\text { Nrf2 nuclear translocation }\end{array}$ & {$[84,85]$} \\
\hline 22 & $\begin{array}{c}\text { R. confervoides } \\
\text { (Rhodophyta, Florideophyceae, Ceramiales) }\end{array}$ & $\begin{array}{c}\text { ABTS }^{+} \text {scavenging: } \mathrm{TEAC}=1.32 \pm 0.02 \mathrm{mM} \\
\text { DPPH scavenging: } \mathrm{IC}_{50}=58.2 \pm 0.4 \mu \mathrm{M}\end{array}$ & [76] \\
\hline
\end{tabular}


Table 2. Cont.

\begin{tabular}{|c|c|c|c|}
\hline Compound & Isolation Source & Assay/Activity & Reference \\
\hline 23 & $\begin{array}{c}\text { P. urceolata } \\
\text { (Rhodophyta, Florideophyceae, Ceramiales) }\end{array}$ & DPPH scavenging: $\mathrm{IC}_{50}=35.8 \mu \mathrm{M}$ & [84] \\
\hline 24 & $\begin{array}{c}\text { R. confervoides, Vertebrata lanosa } \\
\text { (Rhodophyta, Florideophyceae, Ceramiales) }\end{array}$ & $\begin{array}{c}\text { ABTS }^{+} \text {scavenging: TEAC }=1.09 \pm 0.01 \mathrm{mM} \\
\text { CAA } \\
\text { CLPAA } \\
\text { DPPH scavenging: IC } 50=32.0 \pm 0.1 \mu \mathrm{M} \\
\text { ORAC }\end{array}$ & {$[76,86]$} \\
\hline 25 & $\begin{array}{c}\text { S. latiuscula } \\
\text { (Rhodophyta, Florideophyceae, Ceramiales) }\end{array}$ & DPPH scavenging: $\mathrm{IC}_{50}=24.7 \mu \mathrm{M}$ & [74] \\
\hline 26 & $\begin{array}{c}\text { Cladophora wrightiana } \\
\text { (Chlorophyta, Ulvophyceae, Cladophorales) }\end{array}$ & $\begin{array}{c}\text { DPPH scavenging: } 69 \% \text { at } 160 \mu \mathrm{M} \\
\text { OH scavenging } \\
\mathrm{O}_{2}{ }^{-} \text {scavenging } \\
\text { protective effect against UVB-induced apoptosis } \\
\text { and DNA damage in HaCaT cells } \\
\text { scavenging activity against } \mathrm{H}_{2} \mathrm{O}_{2}-\text { or } \\
\text { UVB-generated intracellular ROS in } \mathrm{HaCaT} \text { cells }\end{array}$ & [87] \\
\hline 27 & $\begin{array}{c}\text { R. confervoides } \\
\text { (Rhodophyta, Florideophyceae, Ceramiales) }\end{array}$ & $\begin{array}{c}\text { ABTS }^{+} \text {scavenging: } \mathrm{TEAC}=1.86 \pm 0.02 \mathrm{mM} \\
\text { DPPH scavenging: } \mathrm{IC}_{50}=50.3 \pm 0.3 \mu \mathrm{M}\end{array}$ & [76] \\
\hline 28 & $\begin{array}{c}\text { R. confervoides } \\
\text { (Rhodophyta, Florideophyceae, Ceramiales) }\end{array}$ & $\begin{array}{l}\text { ABTS }^{+} \text {scavenging: } \mathrm{TEAC}=2.11 \mathrm{mM} \\
\text { DPPH scavenging: } \mathrm{IC}_{50}=23.6 \mu \mathrm{M}\end{array}$ & [81] \\
\hline 29 & $\begin{array}{c}\text { R. confervoides } \\
\text { (Rhodophyta, Florideophyceae, Ceramiales) }\end{array}$ & $\begin{array}{l}\text { ABTS }{ }^{+} \text {scavenging: } \mathrm{TEAC}=1.98 \pm 0.01 \mathrm{mM} \\
\text { DPPH scavenging: } \mathrm{IC}_{50}=30.9 \pm 0.1 \mu \mathrm{M}\end{array}$ & [76] \\
\hline 30 & $\begin{array}{c}\text { R. confervoides } \\
\text { (Rhodophyta, Florideophyceae, Ceramiales) }\end{array}$ & $\begin{array}{l}\text { ABTS }{ }^{+} \text {scavenging: } \text { TEAC }=2.35 \pm 0.02 \mathrm{mM} \\
\text { DPPH scavenging: } \mathrm{IC}_{50}=26.3 \pm 0.2 \mu \mathrm{M}\end{array}$ & [76] \\
\hline 31 & $\begin{array}{c}\text { R. confervoides } \\
\text { (Rhodophyta, Florideophyceae, Ceramiales) }\end{array}$ & $\begin{array}{c}\text { ABTS }^{+} \text {scavenging: } \mathrm{TEAC}=2.87 \pm 0.11 \mathrm{mM} \\
\text { DPPH scavenging: } \mathrm{IC}_{50}=19.8 \pm 0.1 \mu \mathrm{M}\end{array}$ & [76] \\
\hline 32 & $\begin{array}{c}\text { R. confervoides } \\
\text { (Rhodophyta, Florideophyceae, Ceramiales) }\end{array}$ & $\begin{array}{l}\text { ABTS }{ }^{+} \text {scavenging: } \text { TEAC }=2.07 \pm 0.12 \mathrm{mM} \\
\text { DPPH scavenging: } \mathrm{IC}_{50}=30.2 \pm 0.2 \mu \mathrm{M}\end{array}$ & [76] \\
\hline 33 & $\begin{array}{c}\text { P. urceolata } \\
\text { (Rhodophyta, Florideophyceae, Ceramiales) }\end{array}$ & DPPH scavenging: $\mathrm{IC}_{50}=16.1 \pm 0.1 \mu \mathrm{M}$ & [88] \\
\hline 34 & $\begin{array}{c}\text { R. confervoides } \\
\text { (Rhodophyta, Florideophyceae, Ceramiales) }\end{array}$ & $\begin{array}{l}\text { ABTS }^{+} \text {scavenging: } \mathrm{TEAC}=2.36 \mathrm{mM} \\
\text { DPPH scavenging: } \mathrm{IC}_{50}=20.8 \mu \mathrm{M}\end{array}$ & [81] \\
\hline 35 & $\begin{array}{c}\text { R. confervoides } \\
\text { (Rhodophyta, Florideophyceae, Ceramiales) }\end{array}$ & $\begin{array}{c}\text { ABTS }^{+} \text {scavenging: } \mathrm{TEAC}=2.11 \pm 0.11 \mathrm{mM} \\
\text { DPPH scavenging: } \mathrm{IC}_{50}=18.6 \pm 0.1 \mu \mathrm{M}\end{array}$ & [76] \\
\hline 36 & $\begin{array}{c}\text { R. confervoides } \\
\text { (Rhodophyta, Florideophyceae, Ceramiales) }\end{array}$ & $\begin{array}{l}\text { ABTS }^{+} \text {scavenging: } \text { TEAC }=1.63 \pm 0.01 \mathrm{mM} \\
\text { DPPH scavenging: } \mathrm{IC}_{50}=50.9 \pm 0.3 \mu \mathrm{M}\end{array}$ & [76] \\
\hline 37 & $\begin{array}{c}\text { R. confervoides } \\
\text { (Rhodophyta, Florideophyceae, Ceramiales) }\end{array}$ & $\begin{array}{l}\text { ABTS }^{+} \text {scavenging: TEAC }=3.68 \pm 0.12 \mathrm{mM} \\
\text { DPPH scavenging: } \mathrm{IC}_{50}=8.72 \pm 0.05 \mu \mathrm{M}\end{array}$ & {$[76]$} \\
\hline 38 & $\begin{array}{c}\text { P. urceolata, R. confervoides } \\
\text { (Rhodophyta, Florideophyceae, Ceramiales) }\end{array}$ & $\begin{array}{c}\text { ABTS }^{+} \text {scavenging: } \text { TEAC }=3.10 \pm 0.13 \mathrm{mM} \\
\text { DPPH scavenging: } \mathrm{IC}_{50}=9.40 \pm 0.05 \\
9.67 \pm 0.04 \mu \mathrm{M}\end{array}$ & {$[76,88]$} \\
\hline 39 & $\begin{array}{c}\text { R. confervoides } \\
\text { (Rhodophyta, Florideophyceae, Ceramiales) }\end{array}$ & $\begin{array}{l}\text { ABTS }{ }^{+} \text {scavenging: TEAC }=3.45 \pm 0.12 \mu \mathrm{mM} \\
\text { DPPH scavenging: } \mathrm{IC}_{50}=7.62 \pm 0.01 \mu \mathrm{M}\end{array}$ & [76] \\
\hline 40 & $\begin{array}{c}\text { S. latiuscula } \\
\text { (Rhodophyta, Florideophyceae, Ceramiales) }\end{array}$ & DPPH scavenging: $\mathrm{IC}_{50}=43.8 \mu \mathrm{M}$ & [82] \\
\hline 41 & $\begin{array}{c}\text { S. latiuscula } \\
\text { (Rhodophyta, Florideophyceae, Ceramiales) }\end{array}$ & DPPH scavenging: $\mathrm{IC}_{50}=8.5 \mu \mathrm{M}$ & [79] \\
\hline
\end{tabular}


Table 2. Cont.

\begin{tabular}{|c|c|c|c|}
\hline Compound & Isolation Source & Assay/Activity & Reference \\
\hline 42 & $\begin{array}{c}\text { R. confervoides } \\
\text { (Rhodophyta, Florideophyceae, Ceramiales) }\end{array}$ & $\begin{array}{l}\text { ABTS }^{+} \text {scavenging: } \mathrm{TEAC}=2.87 \mathrm{mM} \\
\text { DPPH scavenging: } \mathrm{IC}_{50}=5.22 \mu \mathrm{M}\end{array}$ & [81] \\
\hline 43 & $\begin{array}{c}\text { Odonthalia corymbifera } \\
\text { (Rhodophyta, Florideophyceae, Ceramiales) }\end{array}$ & $\begin{array}{c}\text { ABTS }^{+} \text {scavenging: } \mathrm{IC}_{50}=17.3 \pm 0.1 \mu \mathrm{M} \\
\mathrm{Cu}^{2+} \text {-chelation: } \mathrm{IC}_{50}=61.9 \pm 0.1 \mu \mathrm{M} \\
\text { CUPRAC: } \mathrm{EC}_{\mathrm{A} 0.50}=13.6 \pm 0.1 \mu \mathrm{M} \\
\text { DPPH scavenging: } \mathrm{IC}_{50}=24.7 \pm 0.0 \mu \mathrm{M} \\
\text { FRAP: } \mathrm{EC}_{\mathrm{A} 0.50}=11.1 \pm 0.1 \mu \mathrm{M} \\
\text { tyrosinase inhibition: } \mathrm{IC}_{50}=17.3 \pm 0.1 \mu \mathrm{M}\end{array}$ & [89] \\
\hline 44 & $\begin{array}{c}\text { P. morrowii } \\
\text { (Rhodophyta, Florideophyceae, Ceramiales) }\end{array}$ & $\begin{array}{l}\text { LPS-induced ROS generation and ROS-mediated } \\
\text { ERK signaling in RAW } 264.7 \text { macrophages }\end{array}$ & {$[90]$} \\
\hline 45 & $\begin{array}{c}\text { R. confervoides, V. lanosa } \\
\text { (Rhodophyta, Florideophyceae, Ceramiales) }\end{array}$ & $\begin{array}{c}\text { ABTS }^{+} \text {scavenging: } \mathrm{TEAC}=3.05 \mathrm{mM} \\
\text { CAA } \\
\text { CLPAA } \\
\text { DPPH scavenging: } \mathrm{IC}_{50}=17.6 \mu \mathrm{M} \\
\text { ORAC }\end{array}$ & {$[81,86]$} \\
\hline 46 & $\begin{array}{c}\text { S. latiuscula } \\
\text { (Rhodophyta, Florideophyceae, Ceramiales) }\end{array}$ & DPPH scavenging: $\mathrm{IC}_{50}=8.5 \mu \mathrm{M}$ & {$[74]$} \\
\hline 47 & $\begin{array}{c}\text { R. confervoides } \\
\text { (Rhodophyta, Florideophyceae, Ceramiales) }\end{array}$ & $\begin{array}{l}\text { ABTS }^{+} \text {scavenging: } \mathrm{TEAC}=3.18 \mathrm{mM} \\
\text { DPPH scavenging: } \mathrm{IC}_{50}=16.9 \mu \mathrm{M} ; 27 \%\end{array}$ & {$[77,81]$} \\
\hline 48 & $\begin{array}{c}\text { S. latiuscula } \\
\text { (Rhodophyta, Florideophyceae, Ceramiales) }\end{array}$ & DPPH scavenging: $\mathrm{IC}_{50}=8.1 \mu \mathrm{M}$ & {$[74]$} \\
\hline 49 & $\begin{array}{c}\text { Avrainvillea sp. } \\
\text { (Chlorophyta, Ulvophyceae, Bryopsidales) }\end{array}$ & $\begin{array}{c}\text { DPPH scavenging: strong } \\
\text { exogenous ROS scavenging in TPA-treated } \\
\text { HL-60 cells (DCFH-DA): } \mathrm{IC}_{50}=6.1 \mu \mathrm{M}\end{array}$ & [91] \\
\hline 50 & $\begin{array}{c}\text { R. confervoides, V. lanosa } \\
\text { (Rhodophyta, Florideophyceae, Ceramiales) }\end{array}$ & $\begin{array}{c}\text { ABTS }^{+} \text {scavenging: TEAC }=3.16 \mathrm{mM} \\
\text { CAA } \\
\text { CLPAA } \\
\text { DPPH scavenging: } \mathrm{IC}_{50}=19.6 \mu \mathrm{M} \\
\text { ORAC }\end{array}$ & {$[81,86]$} \\
\hline 51 & $\begin{array}{c}\text { R. confervoides } \\
\text { (Rhodophyta, Florideophyceae, Ceramiales) }\end{array}$ & $\begin{array}{l}\text { ABTS }^{+} \text {scavenging: } \mathrm{TEAC}=3.00 \mathrm{mM} \\
\text { DPPH scavenging: } \mathrm{IC}_{50}=14.3 \mu \mathrm{M}\end{array}$ & [81] \\
\hline 52 & $\begin{array}{c}\text { R. confervoides } \\
\text { (Rhodophyta, Florideophyceae, Ceramiales) }\end{array}$ & $\begin{array}{l}\text { ABTS }^{+} \text {scavenging: } \mathrm{TEAC}=2.78 \mathrm{mM} \\
\text { DPPH scavenging: } \mathrm{IC}_{50}=13.8 \mu \mathrm{M}\end{array}$ & {$[81]$} \\
\hline 53 & $\begin{array}{c}\text { S. latiuscula } \\
\text { (Rhodophyta, Florideophyceae, Ceramiales) }\end{array}$ & DPPH scavenging: $\mathrm{IC}_{50}=10.5 \mu \mathrm{M}$ & {$[74]$} \\
\hline 54 & $\begin{array}{c}\text { O. corymbifera } \\
\text { (Rhodophyta, Florideophyceae, Ceramiales) }\end{array}$ & $\begin{array}{c}\text { ABTS }^{+} \text {scavenging: } \mathrm{IC}_{50}=6.7 \pm 0.1 \mu \mathrm{M} \\
\mathrm{Cu}^{2+} \text {-chelation: } \mathrm{IC}_{50}=74.3 \pm 0.1 \mu \mathrm{M} \\
\text { CUPRAC: } \mathrm{EC}_{\mathrm{A} 0.50}=7.8 \pm 0.1 \mu \mathrm{M} \\
\text { DPPH scavenging: } \mathrm{IC}_{50}=13.5 \pm 0.0 \mu \mathrm{M} \\
\text { FRAP: } \mathrm{EC}_{\mathrm{A} 0.50}=10.8 \pm 0.1 \mu \mathrm{M} \\
\text { tyrosinase inhibition: } \mathrm{IC}_{50}=31.0 \pm 0.1 \mu \mathrm{M}\end{array}$ & [90] \\
\hline 55 & $\begin{array}{c}\text { V. lanosa } \\
\text { (Rhodophyta, Florideophyceae, Ceramiales) }\end{array}$ & $\begin{array}{l}\text { CAA } \\
\text { CLPAA } \\
\text { ORAC }\end{array}$ & [86] \\
\hline 56 & $\begin{array}{c}\text { R. confervoides } \\
\text { (Rhodophyta, Florideophyceae, Ceramiales) }\end{array}$ & $\begin{array}{l}\text { ABTS }^{+} \text {scavenging: } \mathrm{TEAC}=3.21 \mathrm{mM} \\
\text { DPPH scavenging: } \mathrm{IC}_{50}=13.6 \mu \mathrm{M}\end{array}$ & [81] \\
\hline 57 & $\begin{array}{c}\text { P. urceolata } \\
\text { (Rhodophyta, Florideophyceae, Ceramiales) }\end{array}$ & DPPH scavenging: $\mathrm{IC}_{50}=19.6 \pm 0.1 \mu \mathrm{M}$ & [88] \\
\hline
\end{tabular}


Table 2. Cont.

\begin{tabular}{|c|c|c|c|}
\hline Compound & Isolation Source & Assay/Activity & Reference \\
\hline 58 & $\begin{array}{c}\text { P. urceolata } \\
\text { (Rhodophyta, Florideophyceae, Ceramiales) }\end{array}$ & DPPH scavenging: $\mathrm{IC}_{50}=21.9 \pm 0.1 \mu \mathrm{M}$ & [88] \\
\hline 59 & $\begin{array}{c}\text { S. latiuscula } \\
\text { (Rhodophyta, Florideophyceae, Ceramiales) }\end{array}$ & DPPH scavenging: $\mathrm{IC}_{50}=10.2 \mu \mathrm{M}$ & [74] \\
\hline 60 & $\begin{array}{c}\text { P. urceolata } \\
\text { (Rhodophyta, Florideophyceae, Ceramiales) }\end{array}$ & DPPH scavenging: $\mathrm{IC}_{50}=8.1 \mu \mathrm{M}$ & {$[84]$} \\
\hline 61 & $\begin{array}{c}\text { P. urceolata } \\
\text { (Rhodophyta, Florideophyceae, Ceramiales) }\end{array}$ & DPPH scavenging: $\mathrm{IC}_{50}=15.1 \mu \mathrm{M}$ & {$[84]$} \\
\hline 62 & $\begin{array}{c}\text { P. urceolata } \\
\text { (Rhodophyta, Florideophyceae, Ceramiales) }\end{array}$ & DPPH scavenging: $\mathrm{IC}_{50}=6.8 \mu \mathrm{M}$ & [84] \\
\hline 63 & $\begin{array}{c}\text { P. urceolata } \\
\text { (Rhodophyta, Florideophyceae, Ceramiales) }\end{array}$ & DPPH scavenging: $\mathrm{IC}_{50}=6.1 \mu \mathrm{M}$ & {$[84]$} \\
\hline 64 & $\begin{array}{c}\text { P. urceolata } \\
\text { (Rhodophyta, Florideophyceae, Ceramiales) }\end{array}$ & DPPH scavenging: $\mathrm{IC}_{50}=7.9 \mu \mathrm{M}$ & [92] \\
\hline 65 & $\begin{array}{c}\text { R. confervoides } \\
\text { (Rhodophyta, Florideophyceae, Ceramiales) }\end{array}$ & $\begin{array}{l}\text { ABTS }{ }^{+} \text {scavenging: } \mathrm{TEAC}=3.58 \mathrm{mM} \\
\text { DPPH scavenging: } \mathrm{IC}_{50}=8.90 \mu \mathrm{M}\end{array}$ & [81] \\
\hline 66 & $\begin{array}{c}\text { Sargassum wightii, Sargassum tenerrimum, } \\
\text { Turbinaria conoides } \\
\text { (Ochrophyta, Phaeophyceae, Fucales) } \\
\text { Ishige okamurae } \\
\text { (Ochrophyta, Phaeophyceae, Ishigeales) } \\
\text { Ecklonia cava } \\
\text { (Ochrophyta, Phaeophyceae, Laminariales) }\end{array}$ & $\begin{array}{c}\text { alkyl scavenging: } \mathrm{IC}_{50}=103.5 \pm 1.9 \mu \mathrm{M} \\
\text { DPPH scavenging: } 64.71-71.07 \% \text { at } 200 \mu \mathrm{g} / \mathrm{mL} \\
\mathrm{H}_{2} \mathrm{O}_{2} \text { scavenging: } 88.33-89.7 \% \text { at } 200 \mu \mathrm{g} / \mathrm{mL} \\
\text { OH scavenging: } \mathrm{IC}_{50}=392.5 \pm 2.8 ; \\
408.5 \pm 3.7 \mu \mathrm{M} \\
\mathrm{O}_{2}{ }^{-} \text {scavenging: } \mathrm{IC}_{50}=115.2 \pm 2.5 ; \\
124.7 \pm 2.4 \mu \mathrm{M} \\
\text { ROO scavenging: } \mathrm{IC}_{50}=128.9 \pm 2.2 \mu \mathrm{M} \\
\text { metal chelating activity: } 11.40-14.38 \% \text { at } \\
200 \mu \mathrm{g} / \mathrm{mL} \\
\mathrm{H}_{2} \mathrm{O}_{2} \text {-induced apoptosis, cytotoxicity, DNA } \\
\text { damage, mitochondrial dysfunction and ROS } \\
\text { generation in HaCaT keratinocytes } \\
\text { intracellular ROS generation (DCFH-DA) in } \\
\text { RAW 264.7 macrophages/V79-4 cells } \\
\text { Nrf2/HO- } 1 \text { signaling pathway in HaCaT } \\
\text { keratinocytes }\end{array}$ & [93-97] \\
\hline 67 & $\begin{array}{c}\text { Gracilaria sp. } \\
\text { (Rhodophyta, Florideophyceae, Gracilariales) }\end{array}$ & $\begin{array}{c}\text { DPPH scavenging: } 83.8 \pm 2.6 \% \\
\text { XO inhibition: } 64.7 \pm 0.7 \%\end{array}$ & [98] \\
\hline 68 & $\begin{array}{c}\text { Sargassum micracanthum } \\
\text { (Ochrophyta, Phaeophyceae, Fucales) }\end{array}$ & $\mathrm{ABTS}^{+}$scavenging: $\mathrm{IC}_{50}=47 \mu \mathrm{M}$ & [99] \\
\hline 69 & $\begin{array}{c}\text { E. cava } \\
\text { (Ochrophyta, Phaeophyceae, Laminariales) }\end{array}$ & $\begin{array}{l}\text { oxidative stress-induced DNA damage in } \\
\text { V79- } 4 \text { cells }\end{array}$ & [100] \\
\hline 70 & $\begin{array}{c}\text { Ishige foliacea } \\
\text { (Ochrophyta, Phaeophyceae, Ishigeales) }\end{array}$ & $\begin{array}{c}\text { enzyme activity (SOD, CAT, GPx) } \\
\text { intracellular ROS generation and lipid } \\
\text { peroxidation in HUVEC/pancreatic } \beta \text { cells } \\
\text { oxidative stress-induced cell death in zebrafish } \\
\text { embryo } \\
\text { streptozotocin-induced pancreatic } \beta \text { cell damage } \\
\text { in rat insulinoma cell line }\end{array}$ & {$[101,102]$} \\
\hline
\end{tabular}


Table 2. Cont.

\begin{tabular}{|c|c|c|c|}
\hline Compound & Isolation Source & Assay/Activity & Reference \\
\hline 71 & $\begin{array}{c}\text { E. cava, Ecklonia kurome, Ecklonia stolonifera, } \\
\text { Eisenia bicyclis } \\
\text { (Ochrophyta, Phaeophyceae, Laminariales) }\end{array}$ & $\begin{array}{c}\text { DPPH scavenging: } \mathrm{IC}_{50}=11.5 ; 22.9 \pm 0.52 ; \\
26 \mu \mathrm{M} \\
\text { OH scavenging: } \mathrm{IC}_{50}=51.8 \pm 2.5 \mu \mathrm{M} \\
\mathrm{O}_{2}-\text { scavenging: } \mathrm{IC}_{50}=26.5 \pm 1.25 ; 107 \mu \mathrm{M} \\
\text { ROO scavenging: } \mathrm{IC}_{50}=28.4 \pm 1.5 \mu \mathrm{M} \\
\text { inhibitory effect on total ROS: } \\
\mathrm{IC}_{50}=4.04 \pm 0.04 \mu \mathrm{M} \\
\text { cellular membrane protein oxidation in RAW } \\
\text { 264.7 macrophages } \\
\text { GSH levels in HepG2 cells } / \mathrm{RAW} \\
264.7 \text { macrophages } \\
\text { HO- } 1 \text { expression } \\
\text { V79-4 cells } \\
\mathrm{H}_{2} \mathrm{O}_{2} \text {-induced lipid peroxidation (TBARS) in } \\
\text { intracellular ROS generation (DCFH-DA) } \\
\text { and oxidative stress induced cell damage in } \\
\text { lung fibroblast cells } \\
\text { MPO activity in HL60 cells } \\
\text { Nrf2 nuclear translocation and activation } \\
\text { PM (particulate matter of less than } 10 \text { mm) } \\
\text {-induced lipid peroxidation and cytokine } \\
\text { expression in human epidermal } \\
\text { keratinocytes }\end{array}$ & [95,103-108] \\
\hline 72 & $\begin{array}{c}\text { E. stolonifera } \\
\text { (Ochrophyta, Phaeophyceae, Laminariales) }\end{array}$ & $\begin{array}{l}\text { DPPH scavenging: } \mathrm{IC}_{50}=8.8 \pm 0.4 \mu \mathrm{M} \\
\text { intracellular ROS scavenging }\end{array}$ & [109] \\
\hline 73 & $\begin{array}{c}\text { I. okamurae } \\
\text { (Ochrophyta, Phaeophyceae, Ishigeales) }\end{array}$ & $\begin{array}{c}\text { alkyl scavenging: } \mathrm{IC}_{50}=18.8 \pm 1.2 \mu \mathrm{M} \\
\text { DPPH scavenging: } \mathrm{IC}_{50}=10.5 \pm 0.5 \mu \mathrm{M} \\
\mathrm{OH} \text { scavenging: } \mathrm{IC}_{50}=27.1 \pm 0.9 \mu \mathrm{M} \\
\mathrm{O}_{2}-\text { scavenging: } \mathrm{IC}_{50}=16.7 \pm 0.6 \mu \mathrm{M} \\
\mathrm{H}_{2} \mathrm{O}_{2} \text {-induced oxidative stress-induced ROS } \\
\text { generation (DCFH-DA) in murine } \\
\text { hippocampal neuronal cells } \\
\text { intracellular Ca }{ }^{2+} \text { level } \\
\text { lipid peroxidation assay (TBARS) } \\
\text { membrane protein oxidation } \\
\text { MPO activity } \\
\mathrm{PM}_{2.5} \text { (fine particulate matter with a } \\
\text { diameter } \leq 2.5 \mu \mathrm{m} \text { ) -induced ROS generation } \\
\text { in human keratinocytes } \\
\mathrm{PM}_{2.5} \text {-induced DNA damage, endoplasmic } \\
\text { reticulum stress and autophagy, } \\
\text { mitochondrial damage, apoptosis via MAPK } \\
\text { signaling pathways }\end{array}$ & {$[97,110,111]$} \\
\hline 74 & $\begin{array}{c}\text { E. cava } \\
\text { (Ochrophyta, Phaeophyceae, Laminariales) }\end{array}$ & $\begin{array}{c}\text { DPPH scavenging: } \mathrm{IC}_{50}=18.6 \pm 1.0 \mu \mathrm{M} \\
\text { OH scavenging: } \mathrm{IC}_{50}=39.6 \pm 2.1 \mu \mathrm{M} \\
\mathrm{O}_{2}{ }^{-} \text {scavenging: } \mathrm{IC}_{50}=21.9 \pm 1.8 \mu \mathrm{M} \\
\text { ROO scavenging: } \mathrm{IC}_{50}=22.7 \pm 1.5 \mu \mathrm{M} \\
\text { cellular membrane protein oxidation in RAW } \\
\text { 264.7 cells } \\
\text { GSH levels in RAW } 264.7 \text { cells } \\
\text { intracellular ROS generation (DCFH-DA) } \\
\text { MPO activity in HL60 cells }\end{array}$ & [95] \\
\hline
\end{tabular}


Table 2. Cont.

\begin{tabular}{|c|c|c|c|}
\hline Compound & Isolation Source & Assay/Activity & Reference \\
\hline 75 & $\begin{array}{l}\text { E. cava, E. kurome, E. stolonifera, E. bicyclis } \\
\text { (Ochrophyta, Phaeophyceae, Laminariales) }\end{array}$ & $\begin{array}{c}\text { DPPH scavenging: } \mathrm{IC}_{50}=6.2 \pm 0.4 ; 8.28 \pm 0.45 \text {; } \\
13 \mu \mathrm{M} \\
\text { OH scavenging: } \mathrm{IC}_{50}=28.6 \pm 2.5 \mu \mathrm{M} \\
\mathrm{O}_{2}{ }^{-} \text {scavenging: } \mathrm{IC}_{50}=7.6 ; 16.2 \pm 1.0 \mu \mathrm{M} \\
\text { ROO scavenging: } \mathrm{IC}_{50}=14.5 \pm 1.8 \mu \mathrm{M} \\
\text { apoptosis in Hep3B cells } \\
\text { cellular membrane protein oxidation in RAW } \\
\text { 264.7 cells } \\
\text { detection of apoptosis-related proteins } \\
\text { GSH levels in RAW } 264.7 \text { cells } \\
\text { intracellular ROS generation (DCFH-DA) in } \\
\text { RAW } 264.7 \text { cells } \\
\text { MPO activity in HL60 cells } \\
\text { PM } \\
\text {-induced lipid peroxidation and cytokine } \\
\text { expression in human epidermal keratinocytes } \\
\text { rotenone-induced oxidative stress in SH-SY5Y } \\
\text { cells }\end{array}$ & $\begin{array}{c}{[95,107-109} \\
112,113]\end{array}$ \\
\hline 76 & $\begin{array}{c}\text { Fucus spiralis } \\
\text { (Ochrophyta, Phaeophyceae, Fucales) }\end{array}$ & DPPH scavenging: $Q_{50}=0.090 \pm 0.002 \mu \mathrm{mol}$ & [114] \\
\hline 77 & $\begin{array}{c}\text { E. cava } \\
\text { (Ochrophyta, Phaeophyceae, Laminariales) }\end{array}$ & $\begin{array}{l}\text { DPPH scavenging: } \mathrm{IC}_{50}=0.60 ; 14.7 \pm 1.2 \mu \mathrm{M} \\
\text { OH scavenging: } \mathrm{IC}_{50}=3.5 \pm 1.55 \mu \mathrm{M} \\
\mathrm{O}_{2}{ }^{-} \text {scavenging: } \mathrm{IC}_{50}=18.6 \pm 1.5 \mu \mathrm{M} \\
\text { ROO scavenging: } \mathrm{IC}_{50}=18.1 \pm 1.0 \mu \mathrm{M} \\
\text { cellular membrane protein oxidation in RAW } \\
\text { 264.7 cells } \\
\text { GSH levels in RAW 264.7 cells } \\
\text { intracellular ROS generation (DCFH-DA) } \\
\text { intracellular ROS detection in UVB-irradiated } \\
\text { HaCaT keratinocytes } \\
\text { MPO activity in HL60 cells }\end{array}$ & {$[95,115,116]$} \\
\hline 78 & $\begin{array}{c}\text { Fucus vesiculosus } \\
\text { (Ochrophyta, Phaeophyceae, Fucales) }\end{array}$ & $\begin{array}{l}\text { DPPH scavenging: } \mathrm{IC}_{50}=16.1 \pm 1.0 \mu \mathrm{M} \\
\mathrm{O}_{2}{ }^{-} \text {scavenging: } \mathrm{IC}_{50}>401.6 \mu \mathrm{M} \\
\text { ORAC: } 3.3 \pm 0.3 \text { units at } 1 \mu \mathrm{g} / \mathrm{mL}\end{array}$ & [117] \\
\hline 79 & $\begin{array}{l}\text { E. cava, E. kurome, E. stolonifera, E. bicyclis } \\
\text { (Ochrophyta, Phaeophyceae, Laminariales) }\end{array}$ & $\begin{array}{c}\text { alkyl scavenging: } \mathrm{IC}_{50}=3.9 \mu \mathrm{M} \\
\text { DPPH scavenging: } \mathrm{IC}_{50}=4.7 \pm 0.3 ; 10.3 ; 12 ; \\
17.7 \pm 0.8 \mu \mathrm{M} \\
\text { OH scavenging: } \mathrm{IC}_{50}=21.4 ; 39.2 \pm 1.8 \mu \mathrm{M} \\
\mathrm{O}_{2}{ }^{-} \text {scavenging: } \mathrm{IC}_{50}=8.4 \mu \mathrm{M} ; \\
\mathrm{IC}_{50}=21.6 \pm 2.2 \mu \mathrm{M} \\
\text { ROO scavenging: } \mathrm{IC}_{50}=21.4 \pm 2.1 \mu \mathrm{M} \\
\text { total ROS generation: } \mathrm{IC}_{50}=3.80 \pm 0.09 \mu \mathrm{M} \\
\text { intracellular ROS generation (DCFH-DA) in } \\
\text { RAW } 264.7 \text { macrophages/Vero cells / zebrafish } \\
\text { system }\end{array}$ & $\begin{array}{c}{[95,105,108,} \\
109,118]\end{array}$ \\
\hline
\end{tabular}


Table 2. Cont.

\begin{tabular}{|c|c|c|c|}
\hline Compound & Isolation Source & Assay/Activity & Reference \\
\hline 80 & $\begin{array}{c}\text { I. okamurae } \\
\text { (Ochrophyta, Phaeophyceae, Ishigeales) } \\
\text { E. cava, E. bicyclis } \\
\text { (Ochrophyta, Phaeophyceae, Laminariales) } \\
\text { Grateloupia elliptica } \\
\text { (Rhodophyta, Florideophyceae, Halymeniales) }\end{array}$ & $\begin{array}{c}\text { ABTS }^{+} \text {scavenging: } \mathrm{IC}_{50}=37.1 \pm 2.8 \mu \mathrm{M} \\
\text { alkyl scavenging: } \mathrm{IC}_{50}=17.3 \pm 1.0 \mu \mathrm{M} \\
\text { DPPH scavenging: } \mathrm{IC}_{50}=8.69 \pm 0.35 ; 9.1 \pm 0.4 ; \\
28 ; 66.5 \pm 0.5 \mu \mathrm{M} \\
\text { OH scavenging: } \mathrm{IC}_{50}=28.7 \pm 1.1 ; 29.7 \pm 1.5 \mu \mathrm{M} \\
\mathrm{O}_{2}{ }^{-} \text {scavenging: } \mathrm{IC}_{50}=15.4 \pm 0.9 ; 15.9 \pm 1.3 \mu \mathrm{M} \\
\text { ROO scavenging: } \mathrm{IC}_{50}=17.1 \pm 2.2 \mu \mathrm{M} \\
\text { singlet oxygen }\left({ }^{1} \mathrm{O}_{2}\right) \text { quenching: } \\
\text { QC }_{50}=30.7 \pm 2.4 \mu \mathrm{M} \\
\text { 264.7 macrophages } \\
\text { cellular membrane protein oxidation in RAW } \\
\text { GSH levels in RAW } 264.7 \text { macrophages } \\
\text { high-glucose-induced oxidative stress } \\
\text { intracellular ROS generation (DCFH-DA) in } \\
\text { UVB-irradiated HaCaT keratinocytes } \\
\text { MPO activity in HL60 cells }\end{array}$ & $\begin{array}{l}{[95,97,119-} \\
121]\end{array}$ \\
\hline 81 & $\begin{array}{c}\text { E. bicyclis } \\
\text { (Ochrophyta, Phaeophyceae, Laminariales) }\end{array}$ & $\begin{array}{c}\text { ABTS }^{+} \text {scavenging: } \mathrm{IC}_{50}=43.3 \pm 2.3 \mu \mathrm{M} \\
\text { DPPH scavenging: } \mathrm{IC}_{50}=103.0 \pm 3.5 \mu \mathrm{M} \\
\text { singlet oxygen }\left({ }^{1} \mathrm{O}_{2}\right) \text { quenching: } \\
\mathrm{QC}_{50}=35.7 \pm 2.4 \mu \mathrm{M}\end{array}$ & [119] \\
\hline 82 & $\begin{array}{c}\text { E. cava, E. kurome, E. bicyclis } \\
\text { (Ochrophyta, Phaeophyceae, Laminariales) }\end{array}$ & $\begin{array}{c}\text { ABTS }{ }^{+} \text {scavenging: } \mathrm{IC}_{50}=43.4 \pm 2.0 \mu \mathrm{M} \\
\text { DPPH scavenging: } \mathrm{IC}_{50}=15.0 ; 95.9 \pm 3.2 \mu \mathrm{M} \\
\mathrm{O}_{2}^{-} \text {scavenging: } \mathrm{IC}_{50}=6.5 \mu \mathrm{M} \\
\text { singlet oxygen }\left({ }^{1} \mathrm{O}_{2}\right) \text { quenching: } \\
\mathrm{QC}_{50}=49.4 \pm 1.7 \mu \mathrm{M} \\
\mathrm{H}_{2} \mathrm{O}_{2} \text {-induced DNA damage } \\
\text { intracellular ROS generation in Vero cells }\end{array}$ & {$[108,119]$} \\
\hline 83 & $\begin{array}{c}\text { E. bicyclis } \\
\text { (Ochrophyta, Phaeophyceae, Laminariales) }\end{array}$ & $\begin{array}{c}\text { DPPH scavenging: } \mathrm{IC}_{50}=0.86 \pm 0.02 \mu \mathrm{M} \\
\text { ONOO }^{-} \text {scavenging: } 1.80 \pm 0.01 \mu \mathrm{M} \\
\text { total ROS: } 6.45 \pm 0.04 \mu \mathrm{M}\end{array}$ & [122] \\
\hline 84 & $\begin{array}{c}\text { E. cava } \\
\text { (Ochrophyta, Phaeophyceae, Laminariales) }\end{array}$ & $\begin{array}{c}\text { alkyl scavenging: } \mathrm{IC}_{50}=2.07 \pm 1.00 \mu \mathrm{M} \\
\text { DPPH scavenging: } \mathrm{IC}_{50}=0.51 \mu \mathrm{M} \\
\mathrm{OH} \text { scavenging: } \mathrm{IC}_{50}=75.6 \mu \mathrm{M} \\
\mathrm{O}_{2}{ }^{-} \text {scavenging: } \mathrm{IC}_{50}=57.2 \mu \mathrm{M} \\
\text { intracellular ROS generation (DCFH-DA) in } \\
\qquad \mathrm{H}_{2} \mathrm{O}_{2} \text {-treated Vero cells }\end{array}$ & [123] \\
\hline 85 & $\begin{array}{c}\text { F. spiralis } \\
\text { (Ochrophyta, Phaeophyceae, Fucales) }\end{array}$ & DPPH scavenging: $Q_{50}=0.087 \pm 0.004 \mu \mathrm{mol}$ & [114] \\
\hline 86 & $\begin{array}{c}\text { F. vesiculosus } \\
\text { (Ochrophyta, Phaeophyceae, Fucales) }\end{array}$ & $\begin{array}{l}\text { DPPH scavenging: } \mathrm{IC}_{50}=19.3 \pm 2.7 \mu \mathrm{M} \\
\mathrm{O}_{2}{ }^{-} \text {scavenging: } \mathrm{IC}_{50}>334.9 \mu \mathrm{M} \\
\text { ORAC: } 3.5 \pm 0.2 \text { units at } 1 \mu \mathrm{g} / \mathrm{mL}\end{array}$ & [117] \\
\hline 87 & $\begin{array}{c}\text { F. vesiculosus } \\
\text { (Ochrophyta, Phaeophyceae, Fucales) }\end{array}$ & $\begin{array}{l}\text { DPPH scavenging: } \mathrm{IC}_{50}=15.8 \pm 1.5 \mu \mathrm{M} \\
\mathrm{O}_{2}{ }^{-} \text {scavenging: } \mathrm{IC}_{50}>175.6 \mu \mathrm{M} \\
\text { ORAC: } 3.2 \pm 0.2 \text { units at } 1 \mu \mathrm{g} / \mathrm{mL}\end{array}$ & [117] \\
\hline 88 & $\begin{array}{c}\text { Acanthophora spicifera } \\
\text { (Rhodophyta, Florideophyceae, Ceramiales) }\end{array}$ & $\begin{array}{l}\text { lipid peroxidation and inhibition of the } \\
\text { generation of MDA in rat liver: } \\
\qquad \mathrm{IC}_{50}=1.0 \times 10^{-2} \mu \mathrm{M}\end{array}$ & [124] \\
\hline
\end{tabular}


Table 2. Cont.

\begin{tabular}{ccc}
\hline Compound & Isolation Source & Assay/Activity \\
\hline $\mathbf{8 9}$ & A. spicifera & lipid peroxidation and inhibition of the \\
& (Rhodophyta, Florideophyceae, Ceramiales) & generation of MDA in rat liver: \\
\hline
\end{tabular}

$\mathrm{ABTS}^{+}:$2,2'-azino-bis (3-ethyl benzothiazoline-6-sulfonic acid) diammonium salt; AChE: acetylcholinesterase; BChE: butyrylcholinesterase; CAA: cellular antioxidant activity; CAT: catalase; CLPAA: cellular lipid peroxidation antioxidant activity; CUPRAC: cupric reducing antioxidant capacity; DCFH-DA: cell-based 2', $7^{\prime}$-dichlorodihydrofluorescein diacetate antioxidant assay; DPPH: 1,1-diphenyl-2-picrylhydrazyl free radical; $\mathrm{EC}_{\mathrm{A} 0.50}$ : effective concentration for absorbance of 0.50; FRAP: ferric reducing antioxidant power; GSH: glutathione; GPx: glutathione peroxidase; $\mathrm{HO}-1$ : heme oxygenase- $1 ; \mathrm{H}_{2} \mathrm{O}_{2}$ : hydrogen peroxide; $\mathrm{IC}_{50}$ : half maximal inhibitory concentration; LPS: lipopolysaccharide; MAPK: mitogen-activated protein kinase; MDA: malondialdehyde; MPO: myeloperoxidase; Nrf2: nuclear factor erythroid 2-related factor 2; $\mathrm{OH}$ : hydroxyl; $\mathrm{ONOO}^{-}$: peroxynitrite; $\mathrm{O}_{2}{ }^{-}$: superoxide anion; $\mathrm{ORAC}$ : oxygen radical absorbance capacity; $\mathrm{Q}_{50}$ : amount of phenolics (in $\mu \mathrm{g}$ ) necessary to obtain $50 \%$ of inhibition in the DPPH assay; $\mathrm{QC}_{50}$ : half maximal quenching concentration; ROO: peroxyl; ROS: reactive oxygen species; SH-SY5Y: human dopaminergic neuronal cell line; SOD: superoxide dismutase; TBARS: thiobarbituric acid reactive substances; TEAC: trolox equivalence antioxidant capacity; TPA: 12-O-tetradecanoylphorbol 13-acetate; V79-4: Chinese hamster lung fibroblast cell line; XO: xanthine oxidase.

Recent studies reveal BPs to be one of the most promising candidates in the prevention of diseases associated with free radical attack [73]. Hitherto, more than 60 BPs, mainly isolated from marine red algae, have been reported to exert antioxidant activity in vitro. Their antioxidant activity has been primarily determined by the DPPH radical scavenging method. In general, the BPs shown in Table 2 exhibited better activity than that of butylated hydroxytoluene $\left(\mathrm{BHT}, \mathrm{IC}_{50}=82.1 \mu \mathrm{M}\right)$, a synthetic antioxidant often used as positive control, with BPs isolated from the red algae Polysiphonia urceolata, Rhodomela confervoides and Symphyocladia latiuscula, as well as the green alga Avrainvillea sp. possessing the highest activities in the DPPH assay $\left(\mathrm{IC}_{50}<10.0 \mu \mathrm{M}\right)$.

Previous studies have shown that the non-brominated phenolic compounds 5-(hydroxylmethyl)-2-methoxybenzene-1,3-diol (2) and 3,4-dihydroxy-benzoic acid (DBA, 26) exert antioxidant activity $[75,77,87]$. Specifically, DBA (26) was found to reduce the levels of intracellular ROS generated by $\mathrm{H}_{2} \mathrm{O}_{2}$ or UVB treatment of the human $\mathrm{HaCaT}$ keratinocytes cells, thus protecting the cells from UVB-induced oxidative stress [87].

A series of BPs $(3,4,6,9-11,13-16,18,19,21,22,24,27-32,34-39,42,45,47,50-52$, $56,65)$ have been isolated from the red alga $R$. confervoides $[76,77,81,83,85]$. Among them, compounds 10, 15, 16, 18, 19, 37, 38, 39, 42, and 65 exerted the highest antioxidant activity with $\mathrm{IC}_{50}$ values of $7.43,5.43,5.70,3.82,9.52,8.72,9.40,7.62,5.22$, and $8.90 \mu \mathrm{M}$, respectively, with all but 37 and 38 bearing a 2,3-dibromo-4,5-dihydroxy-benzyl skeleton. In particular, rhodomelin A (18) displayed the highest scavenging activity towards DPPH radical with an $\mathrm{IC}_{50}$ value of $3.82 \mu \mathrm{M}$. The 2,3-dibromo-4,5-dihydroxy-benzyl unit appears to be a structural element positively influencing antioxidant activity. Moreover, it seems that the antioxidant activity may have a close connection to the number of hydroxyl groups in the molecule [74]. Additionally, the presence of two successive hydroxyl groups in the benzene ring appears to be necessary for increased levels of antioxidant activity to be displayed. This conclusion is supported by the higher $\mathrm{IC}_{50}$ values exerted by BPs lacking the second free hydroxyl group by having a methoxyl group instead (e.g., 3, 22, 27, and 36 with $\mathrm{IC}_{50}$ values of 50.6, 58.2, 50.3 and $50.9 \mu \mathrm{M}$, respectively). For example, compound 22, with an $\mathrm{IC}_{50}$ value of $58.2 \mu \mathrm{M}$, bearing only one hydroxyl substituent, is less active than 21 $\left(\mathrm{IC}_{50}=20.3 \mu \mathrm{M}\right)$ that possesses the characteristic 4,5-dihydroxy-benzyl group [84,88]. On the other hand, a short and hydrophilic side chain leads to lower activities (e.g., compounds $4,6,9$, and 11 with $\mathrm{IC}_{50}$ values of $42.3,40.5,38.4$, and $22.5 \mu \mathrm{M}$, as compared to compounds $10,13,14,15,16,18$, and 19 with $\mathrm{IC}_{50}$ values of $7.43,12.4,14.6,5.43,5.70,3.82$, and $9.52 \mu \mathrm{M}$, respectively). 
<smiles>Cc1c(Br)c(O)c(O)c(Br)c1Br</smiles>

1<smiles>[R]Oc1c(O)cc(CO)c([R])c1[R]</smiles>

$2 \mathrm{R}_{1}=\mathrm{R}_{3}=\mathrm{H}, \mathrm{R}_{2}=\mathrm{OMe}$

$3 \mathrm{R}_{1}=\mathrm{H}, \mathrm{R}_{2}=\mathrm{Br}, \mathrm{R}_{3}=\mathrm{Me}$

$4 \mathrm{R}_{1}=\mathrm{R}_{2}=\mathrm{Br}, \mathrm{R}_{3}=\mathrm{H}$<smiles>[R20]Cc1c([R])c([R2])c(O)c([R])c1Br</smiles>

$5 \mathrm{R}_{1}=\mathrm{OH}, \mathrm{R}_{2}=\mathrm{H}, \mathrm{R}_{3}=\mathrm{Br}, \mathrm{R}_{4}=\mathrm{Me}$

$6 \mathrm{R}_{1}=\mathrm{Br}, \mathrm{R}_{2}=\mathrm{OH}, \mathrm{R}_{3}=\mathrm{H}, \mathrm{R}_{4}=\mathrm{Me}$

$7 \mathrm{R}_{1}=\mathrm{R}_{3}=\mathrm{Br}, \mathrm{R}_{2}=\mathrm{OH}, \mathrm{R}_{4}=\mathrm{Me}$

$8 \mathrm{R}_{1}=\mathrm{OH}, \mathrm{R}_{2}=\mathrm{R}_{3}=\mathrm{Br}, \mathrm{R}_{4}=\mathrm{H}$<smiles>CCOCc1cc(O)c(O)c(Br)c1Br</smiles><smiles>OCC(O)COCc1cc(O)c(O)c(Br)c1Br</smiles>

10<smiles>NCc1cc(O)c(O)c(Br)c1Br</smiles>

11<smiles>O=C1CCCN1Cc1c(Br)c(O)c(O)c(Br)c1Br</smiles>

12<smiles>O=C(O)C1CCC(=O)N1Cc1cc(O)c(O)c(Br)c1Br</smiles>

$13 \mathrm{R}=\mathrm{H}$

$14 \mathrm{R}=\mathrm{Me}$<smiles>[R]OC(=O)CCC(=O)NCc1cc(O)c(O)c(Br)c1Br</smiles>

$15 \mathrm{R}=\mathrm{H}$

$16 \mathrm{R}=\mathrm{Me}$<smiles></smiles><smiles>[R20]c1cc(C=O)cc(Br)c1[R20]</smiles>

$21 \mathrm{R}_{1}=\mathrm{H}, \mathrm{R}_{2}=\mathrm{OH}$

$22 \mathrm{R}_{1}=\mathrm{Me}, \mathrm{R}_{2}=\mathrm{OH}$

$23 \mathrm{R}_{1}=\mathrm{H}, \mathrm{R}_{2}=\mathrm{Br}$<smiles>COc1c(O)cc(C(N)=O)cc1Br</smiles>

28<smiles>[R]c1c(O)c(O)c(Br)c(Br)c1C=O</smiles>

$24 \mathrm{R}=\mathrm{H}$

$25 \mathrm{R}=\mathrm{Br}$<smiles>OCCc1cc(O)c(O)c(Br)c1Br</smiles>

29<smiles>[R]Oc1c([R])cc(C(=O)O)cc1[R]</smiles>

$26 \mathrm{R}_{1}=\mathrm{OH}, \mathrm{R}_{2}=\mathrm{R}_{3}=\mathrm{H}$

$27 \mathrm{R}_{1}=\mathrm{Br}, \mathrm{R}_{2}=\mathrm{Me}, \mathrm{R}_{3}=\mathrm{OH}$<smiles>[R]OC(=O)Cc1cc(O)c([R6])c(Br)c1[R2]</smiles>

$30 \mathrm{R}_{1}=\mathrm{R}_{2}=\mathrm{H}, \mathrm{R}_{3}=\mathrm{Me}$

$31 \mathrm{R}_{1}=\mathrm{R}_{3}=\mathrm{H}, \mathrm{R}_{2}=\mathrm{Br}$

$32 \mathrm{R}_{1}=\mathrm{R}_{3}=\mathrm{Me}, \mathrm{R}_{2}=\mathrm{H}$

Figure 2. Chemical structures of compounds 1-32. 
<smiles>CCCCOC(=O)Cc1cc(Br)c(O)c(Br)c1</smiles>

33<smiles>COc1c(O)cc(CC(N)=O)cc1Br</smiles>

34<smiles>COC(C=O)Cc1cc(O)c(O)c(Br)c1Br</smiles>

35<smiles>COc1c(O)cc(CC(N)C(=O)O)cc1Br</smiles><smiles>[R]c1c(/C=C/C(C)=O)cc(O)c(O)c1[R]</smiles>

$37 \mathrm{R}_{1}=\mathrm{Br}, \mathrm{R}_{2}=\mathrm{H}$ $38 \mathrm{R}_{1}=\mathrm{H}, \mathrm{R}_{2}=\mathrm{Br}$ $39 \mathrm{R}_{1}=\mathrm{R}_{2}=\mathrm{Br}$<smiles>O=C1CCCC[C@H]1Cc1c(Br)c(O)c(O)c(Br)c1Br</smiles>

41<smiles>O=C1C[C@H](Cc2cc(O)c(O)c(Br)c2Br)C(=O)N1</smiles>

42<smiles>COC(=O)C(Cc1cc(Br)c(O)c(O)c1Br)/C(=C/C(=O)O)C(=O)O</smiles>

40<smiles>O=C1C=C(CO)C(=O)[C@H]1Cc1cc(O)c(O)c(Br)c1Br</smiles>

43<smiles>Oc1cc(COCc2cc(O)c(O)c(Br)c2)cc(Br)c1O</smiles>

44<smiles>[R2]c1c(O)c(O)c(Br)c(Br)c1COCc1c([R])c(Br)c(O)c(Br)c1Br</smiles>

$45 \mathrm{R}_{1}=\mathrm{R}_{2}=\mathrm{H}$ $46 R_{1}=R_{2}=B r$<smiles>[R2]c1c(O)c(O)c(O)c(Br)c1Cc1c([R2])c(Br)c(Br)c(O)c1Br</smiles>

$47 \mathrm{R}_{1}=\mathrm{R}_{2}=\mathrm{H}$

$48 \mathrm{R}_{1}=\mathrm{R}_{2}=\mathrm{Br}$<smiles>[R7]Cc1c([R2])c(O)c(O)c(Br)c1Cc1c([R3])c(O)c(O)c(Br)c1Br</smiles>

$52 R_{1}=E t, R_{2}=R_{3}=H$ $53 \mathrm{R}_{1}=\mathrm{Me}, \mathrm{R}_{2}=\mathrm{R}_{3}=\mathrm{Br}$<smiles>OCc1cc(Br)c(O)c(O)c1Cc1ccc(O)c(Br)c1</smiles>

49<smiles>[R]Cc1cc(O)c(O)c(Br)c1Cc1cc(O)c(O)c(Br)c1Br</smiles>

$50 \mathrm{R}=\mathrm{H}$

$51 \mathrm{R}=\mathrm{Me}$<smiles>COCc1cc(O)c(Oc2c(Cc3c(COC)cc(O)c(O)c3Br)cc(O)c(O)c2Br)c(Br)c1Br</smiles>

Figure 3. Chemical structures of compounds 33-54. 


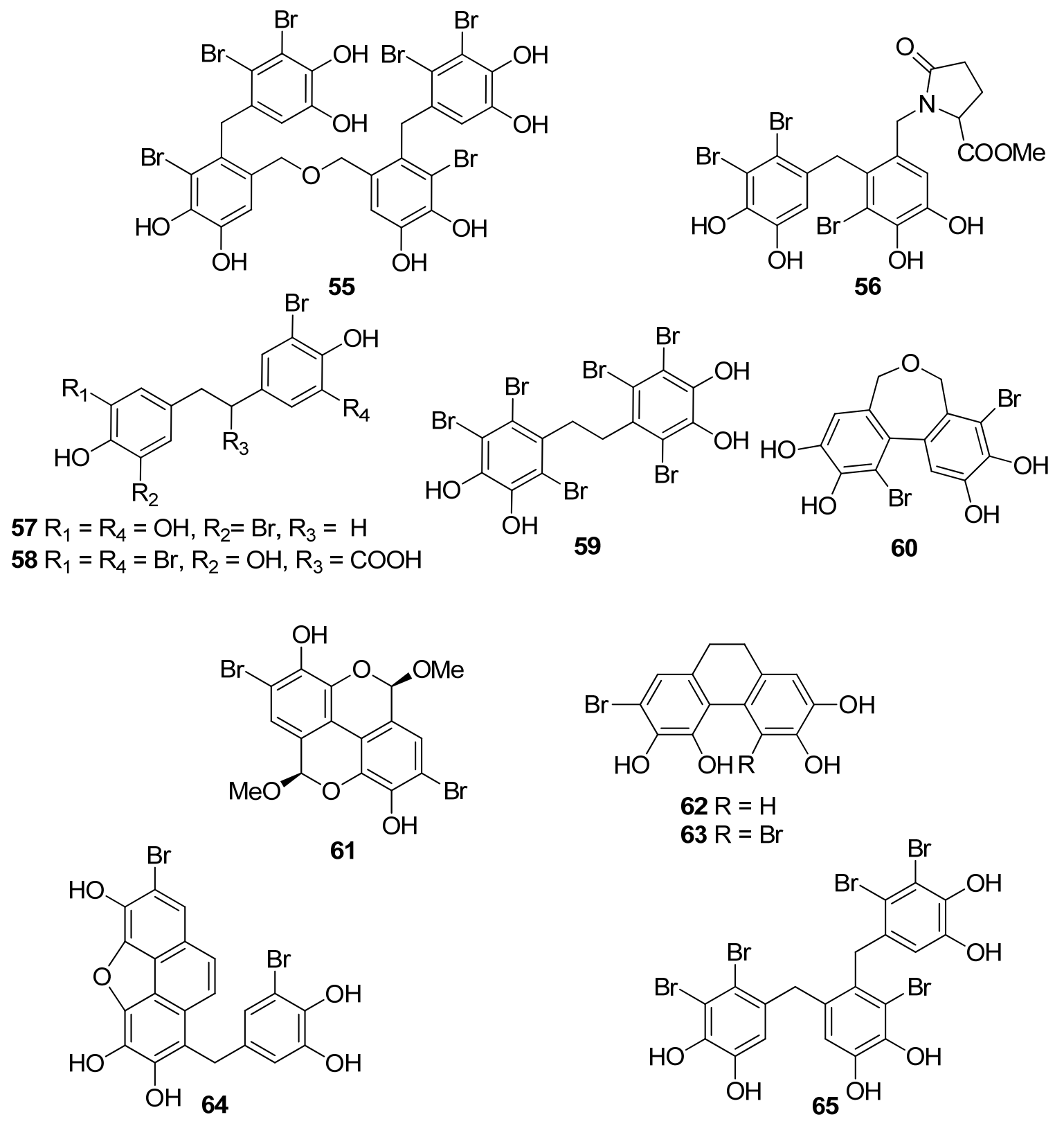

Figure 4. Chemical structures of compounds 55-65.

Ryu et al. (2019) found that 3-bromo-4,5-dihydroxy-benzaldehyde (21) protects human keratinocytes from oxidative stress by upregulating extracellular signal-regulated kinase (ERK) and protein kinase B (Akt), which allows nuclear factor erythroid 2-related factor 2 (Nrf2) to induce the transcription of the antioxidant enzyme heme oxygenase (HO-1) [85].

BPs 8 and 41, as well as the biphenyl BPs $46,48,53$, and 59 , isolated from the red alga $S$. latiuscula, all being fully substituted, showed particularly high radical scavenging activity, with $\mathrm{IC}_{50}$ values of $7.5,8.5,8.5,8.1,10.5$, and $10.2 \mu \mathrm{M}$, respectively, significantly higher than that of $\mathrm{L}$-ascorbic acid $\left(\mathrm{IC}_{50}=15.3 \mu \mathrm{M}\right)$, employed as positive control $[74,79]$. The structurally similar avrainvilleol (49), isolated from the green alga Avrainvillea sp., also exerted high antioxidant activity with an $\mathrm{IC}_{50}$ value of $6.1 \mu \mathrm{M}$ [91]. The DPPH radicalscavenging activities of the bis-phenols $46,48,53$, and 59 are noticeably higher than those of the mono-phenols $\mathbf{1}, \mathbf{7}, \mathbf{8}, \mathbf{1 2}, \mathbf{2 0}, \mathbf{2 5}$, and $\mathbf{4 1}$ with $\mathrm{IC}_{50}$ values of $14.0,15.5,7.5,14.4,24.0$, 24.7 , and $8.5 \mu \mathrm{M}$, respectively. Apparently, DPPH scavenging activity is directly related to the overall number of phenol units in the molecules (e.g., 45 and 47 vs. 65 and 14 vs. 56, with $\mathrm{IC}_{50}$ values of 17.6 and 16.9 vs. 8.90 , and 18.5 vs. $13.6 \mu \mathrm{M}$, respectively). Compounds having the same number of phenolic hydroxyl groups, such as compounds $\mathbf{2 8}$ and $\mathbf{3 4}$, or $\mathbf{4 5}$ 
and 47 exhibit similar DPPH radical scavenging activity (23.6 and 20.8, or 17.6 and $16.9 \mu \mathrm{M}$, respectively) [76,81].<smiles>Oc1cc(O)cc(O)c1</smiles>

66<smiles>COc1ccc(C(=O)CC(O)c2c(O)cc(O)cc2O)cc1</smiles>

67

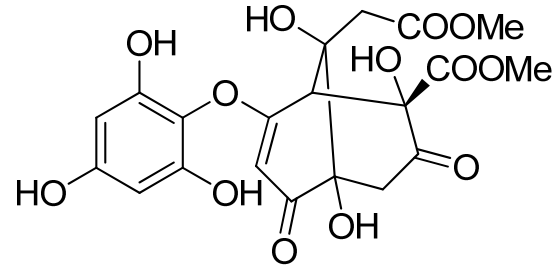

68<smiles>Oc1cc(O)cc(Oc2c(O)cc(O)cc2Oc2c(O)cc(Oc3c(O)cc(Oc4c(O)cc(Oc5c(O)cc(Oc6c(O)cc(Oc7cc(O)cc(O)c7Oc7cc(O)cc(O)c7Oc7cc(O)cc(O)c7)cc6O)cc5O)cc4O)cc3O)cc2O)c1</smiles>

70

Figure 5. Chemical structures of compounds 66-70.

Furthermore, a series of BPs isolated from the red alga P. urceolata $(23,33,38,57$, 58, 60-64) was shown to exhibit significant DPPH radical scavenging activity [84,88,92]. Among them, compounds 60, 62, 63, and 64, bearing four hydroxyl groups in their molecules, were the most active with $\mathrm{IC}_{50}$ values of $8.1,6.8,6.1$, and $7.9 \mu \mathrm{M}$, respectively. Moreover, in this case, the necessity for the presence of two successive hydroxyl groups in the benzene ring is evident for the display of enhanced antioxidant activity. Another important factor for enhanced activity is the conjugation of the benzene rings, as evidenced by comparing compounds 57 and 63 . The conjugation in the dihydrophenanthrene skeleton results to a reduction in the $\mathrm{IC}_{50}$ values from $19.6 \mu \mathrm{M}$ for 57 to $6.1 \mu \mathrm{M}$ for 63 .

The degree of bromination does not appear to affect the antioxidant activity in a consistent manner. For example, in the case of BPs $\mathbf{1 2}$ and $\mathbf{1 3}$ the $\mathrm{IC}_{50}$ values were comparable (14.4 and $12.4 \mu \mathrm{M}$, respectively). In the case of 19 and 20 ( $\mathrm{IC}_{50}$ values 9.52 and $24.0 \mu \mathrm{M}$, respectively), it appears that the extra bromine atom in $\mathbf{2 0}$ reduces the antioxidant activity, while in the cases of 24 and 25, 45, and 46, as well as 47 and 48 it appears that the presence of an additional bromine atom increases the activity. Moreover, by comparing the $\mathrm{IC}_{50}$ values of 37 and 38 (8.72 and $9.40 \mu \mathrm{M}$, respectively), it appears that the site of bromination is of no decisive importance.

Choi et al. (2018) showed that bis (3-bromo-4,5-dihydroxybenzyl) ether (BDDE, 44), isolated from Polysiphonia morrowii, suppresses the lipopolysaccharide (LPS)-induced ROS generation in RAW 264.7 macrophage cells. In turn, inhibition of LPS-induced ROS generation by BDDE (44) caused ERK inactivation and an inflammatory reaction [90]. Therefore, BBDE (44) inhibits LPS-induced inflammation by inhibiting the ROS-mediated ERK signal- 
ing pathway in RAW 264.7 macrophage cells and thus can be useful for the treatment of inflammatory diseases [90].

Phlorotannins, exclusively found in macroalgae, are oligomers or polymers of phloroglucinol (1,3,5-trihydroxybenzene, PGU, 66) that can be classified according to the linkage of PGU units [125,126]. Park et al. (2019) suggested that PGU (66) is able to protect HaCaT keratinocytes against oxidative stress-induced DNA damage and apoptosis through the activation of the Nrf2/HO-1 signaling pathway [96].<smiles>Oc1cc(O)cc(Oc2c(O)cc(O)c3c2Oc2c(O)cc(O)cc2O3)c1</smiles>

71<smiles>Oc1cc(O)cc(Oc2c(O)cc3c(c2O)Oc2cc(Oc4c(O)cc(O)cc4O)c(O)c(O)c2O3)c1</smiles><smiles></smiles>

72<smiles></smiles><smiles>Oc1cc(O)cc(Oc2c(O)cc(O)c3c2Oc2c(O)cc(Oc4c(O)cc(Oc5c(O)cc(O)c6c5Oc5c(O)cc(O)cc5O6)cc4O)cc2O3)c1</smiles>

Figure 6. Chemical structures of compounds 71-76.

Until now, numerous phlorotannins purified from brown seaweeds, especially from Ecklonia sp., have been proven to exert antioxidant activities and protective effects against $\mathrm{H}_{2} \mathrm{O}_{2}$-induced cell damage [93,95,104-106,108,110]. In particular, eckol (71), eckstolonol (72), diphlorethohydroxycarmalol (DPHC, 73), 7-phloroglucinol-eckol (74), dieckol (75), fucodiphloroethol G (77), phlorofucofuroeckol-A (79) 6,6'-bieckol (80), 6,8'-bieckol (81), 8,8'-bieckol (82), 974-B (83), and 2,7'-phloroglucinol-6,6'-bieckol (84), isolated from Eisenia bicyclis, Ecklonia cava, Ecklonia stolonifera, and Ishige okamurae, have shown potent antioxidant 
activity as determined by the DPPH radical scavenging method, with $\mathrm{IC}_{50}$ values of $11.5,8.8$, $10.5,18.6,6.2,0.60,4.7,8.69,15.0,0.86$, and $0.51 \mu \mathrm{M}$, respectively $[95,97,104,108,109,115,122,123]$. Among them, fucodiphloroethol G (77), compound 83, and 2,7"-phloroglucinol-6,6'-bieckol (84) are the most effective, with $\mathrm{IC}_{50}$ values in the nanomolar range $[115,122,123]$.<smiles>Oc1cc(O)c(Oc2cc(O)cc(O)c2Oc2cc(O)cc(O)c2-c2c(O)cc(O)cc2O)c(O)c1</smiles>

77<smiles>Oc1cccc(Oc2c(O)cc(O)c3c2Oc2c(O)cc4oc5c(Oc6cccc(O)c6)c(O)cc(O)c5c4c2O3)c1</smiles>

79<smiles>Oc1cc(O)c(Oc2cc(O)c(Oc3cc(O)c(Oc4cc(O)c(-c5c(O)cc(O)cc5O)c(O)c4)c(O)c3)c(O)c2)c(O)c1</smiles>

78

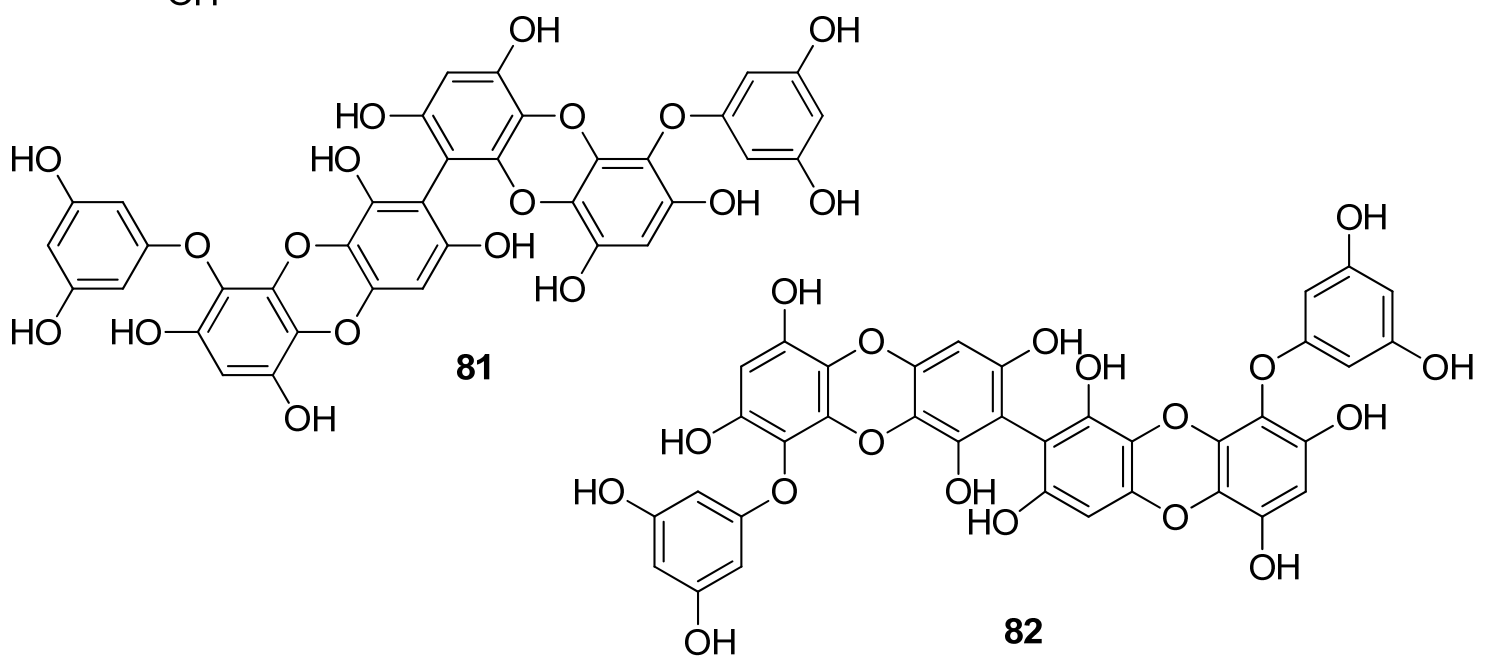

Figure 7. Chemical structures of compounds 77-82. 


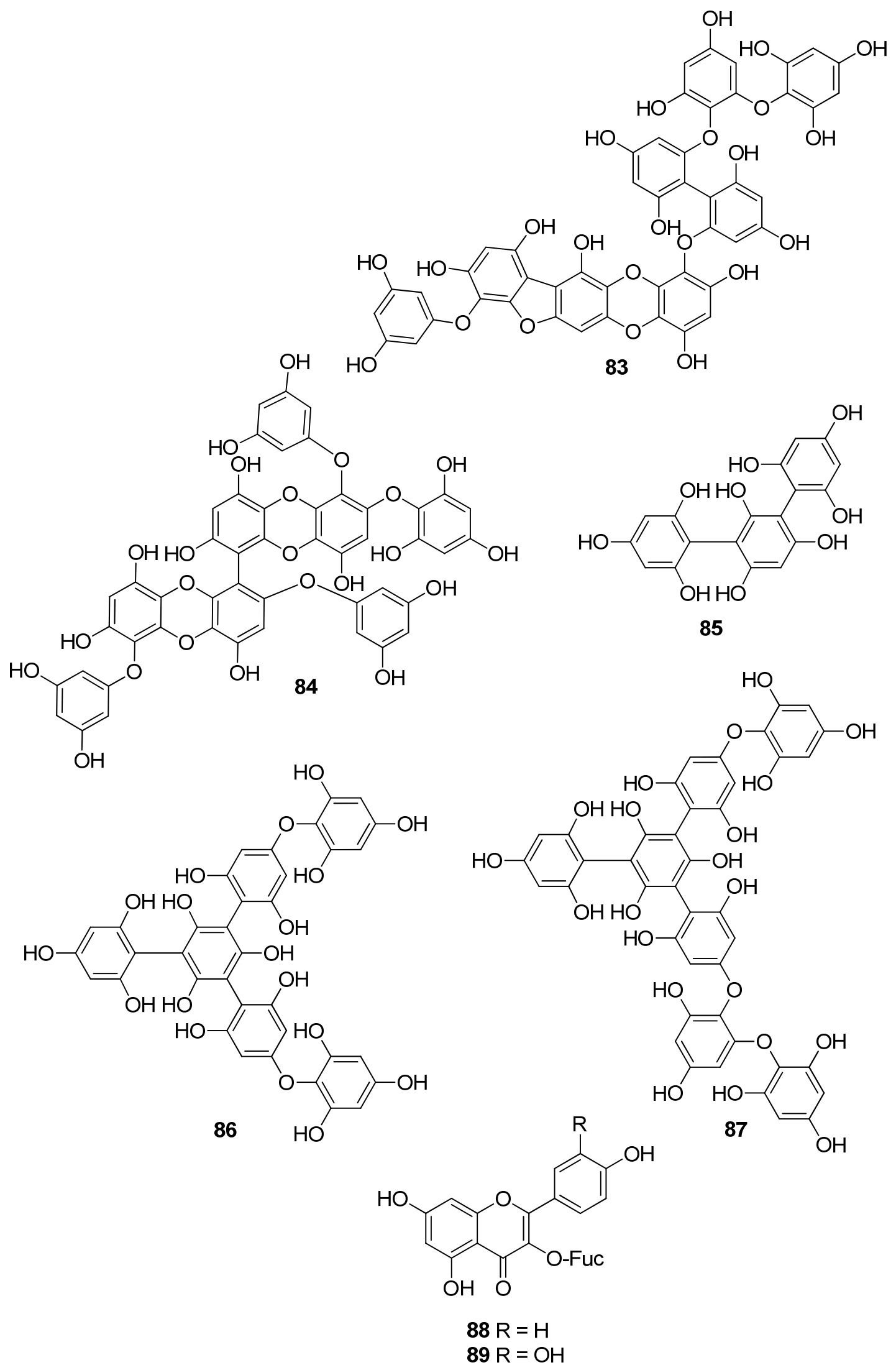

Figure 8. Chemical structures of compounds 83-89. 
Eckol (71) suppresses the production of intracellular ROS and increases GSH levels in HepG2 cells [103], while dieckol (75) induces apoptosis in human hepatocellular carcinoma Hep3B cells via the activation of both death receptor and mitochondrial-dependent pathways, by activating caspases-3, -7, -8, -9, and poly(ADP-ribose) polymerase (PARP) [113]. Moreover, eckol (71), phlorofucofuroeckol A (79), dieckol (75), and 8,8'-bieckol (82) have shown potent inhibition of phospholipid peroxidation at a concentration of $1 \mu \mathrm{M}$ in a liposome system [108]. Lee et al. (2018) showed that both eckol (71) and dieckol (75) attenuated $\mathrm{PM}_{10}$ (particulate matter of less than $10 \mathrm{~mm}$ ) -induced lipid peroxidation and cytokine expression in human epidermal keratinocytes [107]. Similarly, Zhen et al. (2019) showed that DPHC (73) blocked $\mathrm{PM}_{2.5}$ (fine particulate matter with a diameter $\leq 2.5 \mu \mathrm{m}$ ) -induced ROS generation in human keratinocytes [111]. Specifically, DPHC (73) protected cells against $\mathrm{PM}_{2.5}$-induced DNA damage, endoplasmic reticulum stress, and autophagy, and inhibited lipid peroxidation, protein carbonylation, and increased epidermal height in HR-1 hairless mice exposed to $\mathrm{PM}_{2.5}$. Moreover, DPHC (73) attenuated $\mathrm{PM}_{2.5}$-induced apoptosis and mitogen-activated protein kinase (MAPK) protein expression [111]. In the study of Heo et al. (2012), the neuroprotective effect of DPHC (73) against $\mathrm{H}_{2} \mathrm{O}_{2}$-induced oxidative stress in murine hippocampal neuronal cells HT22 was investigated and it was found that DPHC protected cells from $\mathrm{H}_{2} \mathrm{O}_{2}$-induced neurotoxicity by restoring cell viability [110]. Specifically, DPHC (73) slightly reduced the expression of Bax induced by $\mathrm{H}_{2} \mathrm{O}_{2}$, but recovered the expression of $\mathrm{Bcl}-\mathrm{xL}$, as well as caspase- 9 and -3 mediated PARP cleavage by $\mathrm{H}_{2} \mathrm{O}_{2}$, while it effectively inhibited intracellular ROS and lipid peroxidation in a dose-dependent manner and suppressed the elevation of $\mathrm{H}_{2} \mathrm{O}_{2}$-induced $\mathrm{Ca}^{2+}$ release [110].

On the other hand, the protective effects of 6,6'-bieckol (80) against high-glucoseinduced oxidative stress were investigated using human umbilical vein endothelial cells (HUVECs) susceptible to oxidative stress [121]. It was found that 6,6'-bieckol (80) significantly inhibited the high-glucose treatment-induced HUVECs' cell death. Moreover, compound $\mathbf{8 0}$ dose-dependently decreased thiobarbituric acid reactive substances (TBARS), intracellular ROS generation, and nitric oxide levels that were increased by high glucose. High glucose levels induced the overexpression of inducible nitric oxide synthase (iNOS), cyclooxygenase 2 (COX-2), and nuclear factor-kappa B (NF-kB) proteins in HUVECs, but treatment with 6,6'-bieckol (80) reduced their overexpression.

The structure-activity relationship of phlorotannins, although not fully elucidated, suggests that the hydroxyl group availability influences phlorotannins' antioxidant capacity to a far greater extent than polymerization and the size of the molecule.

Flavonoids are another important class of polyphenolic secondary metabolites often exhibiting potent antioxidant activity, found predominantly in plants and fungi, but also to a lesser degree in algae. The flavonoids acanthophorin A (88) and acanthophorin B (89), isolated from the red alga Acanthophora spicifera, were shown to exert significant antioxidant activity by preventing lipid peroxidation and inhibiting the generation of MDA in liver homogenates of rat in vitro. Compounds 88 and 89, with $\mathrm{IC}_{50}$ values $1.0 \times 10^{-2}$ and $1.5 \times 10^{-2} \mu \mathrm{M}$, respectively, displayed almost 10,000 times higher activity than vitamin $\mathrm{E}$ $\left(\mathrm{IC}_{50}=160 \mu \mathrm{M}\right)$ [124].

\section{Terpenoids}

Terpenoids, also called isoprenoids, represent a diverse class of naturally occurring secondary metabolites composed of isoprene units. Terpenoids, often possessing multicyclic structures with various functional groups [127], are ubiquitous, found in almost all classes of living organisms, including macroalgae. Table 3 presents the terpenoids possessing significant antioxidant activities isolated so far from marine macroalgae (Figures 9-12). 
Table 3. Terpenoids from macroalgae with antioxidant activity.

\begin{tabular}{|c|c|c|c|}
\hline Compound & Isolation Source & Assay/Activity & Reference \\
\hline 90 & $\begin{array}{c}\text { Plocamium sp. } \\
\text { (Rhodophyta, Florideophyceae, Plocamiales) }\end{array}$ & $\begin{array}{c}\text { DPPH scavenging: } \mathrm{IC}_{50}=0.05 \pm 0.01 \mathrm{mM} \\
\mathrm{H}_{2} \mathrm{O}_{2} \text { scavenging: } \mathrm{IC}_{50}=5.58 \pm 1.11 \mathrm{mM} \\
\mathrm{NO} \text { scavenging: } \mathrm{IC}_{50}=4.18 \pm 0.22 \mathrm{mM} \\
\text { reducing power }\left(\mathrm{Fe}^{3+} \text { to } \mathrm{Fe}^{2+} \text { reduction) }\right.\end{array}$ & [128] \\
\hline 91 & $\begin{array}{c}\text { Ulva fasciata } \\
\text { (Chlorophyta, Ulvophyceae, Ulvales) }\end{array}$ & $\begin{array}{l}\text { ABTS }+ \text { scavenging: } 66.8 \pm 1.5 \% \text { at } 50 \mu \mathrm{M} \\
\text { DPPH scavenging: } \mathrm{IC}_{50}=13.74 \pm 1.38 \mathrm{mM}\end{array}$ & [129] \\
\hline 92 & $\begin{array}{c}\text { Pyropia orbicularis } \\
\text { (Rhodophyta, Bangiophyceae, Bangiales) }\end{array}$ & $\begin{array}{c}\text { activation of antioxidant responses during } \\
\text { desiccation }\end{array}$ & [130] \\
\hline 93 & $\begin{array}{c}\text { U. fasciata } \\
\text { (Chlorophyta, Ulvophyceae, Ulvales) }\end{array}$ & $\begin{array}{c}\text { ABTS }^{+} \text {scavenging } \\
\text { DPPH scavenging: } \mathrm{IC}_{50}=80.56 \pm 2.43 \mathrm{mM}\end{array}$ & [129] \\
\hline 94 & $\begin{array}{c}\text { U. fasciata } \\
\text { (Chlorophyta, Ulvophyceae, Ulvales) }\end{array}$ & $\begin{array}{c}\text { ABTS }^{+} \text {scavenging } \\
\text { DPPH scavenging: } \mathrm{IC}_{50}=23.60 \pm 1.15 \mathrm{mM}\end{array}$ & [129] \\
\hline 95 & $\begin{array}{c}\text { U. fasciata } \\
\text { (Chlorophyta, Ulvophyceae, Ulvales) }\end{array}$ & $\begin{array}{c}\text { ABTS }^{+} \text {scavenging } \\
\text { DPPH scavenging: } \mathrm{IC}_{50}=20.83 \pm 0.92 \mathrm{mM}\end{array}$ & [129] \\
\hline 96 & $\begin{array}{c}\text { U. fasciata } \\
\text { (Chlorophyta, Ulvophyceae, Ulvales) }\end{array}$ & $\begin{array}{c}\text { ABTS }{ }^{+} \text {scavenging: } 78 \pm 1.9 \% \text { at } 50 \mu \mathrm{M} \\
\text { DPPH scavenging: } \mathrm{IC}_{50}=10.24 \pm 0.98 \mathrm{mM}\end{array}$ & [129] \\
\hline 97 & $\begin{array}{c}\text { Laurencia tristicha } \\
\text { (Rhodophyta, Florideophyceae, Ceramiales) }\end{array}$ & $\begin{array}{c}\text { alcohol-induced oxidative injury in rats } \\
\text { enzyme activity (SOD, CAT, GPx) } \\
\text { D-galactose-induced oxidation in mice } \\
\text { endogenous apoptosis-related genes' expression } \\
\text { (BAX, cytochrome c, cytochrome P450, BCL-2, } \\
\text { Caspase-9 and Caspase-3) } \\
\text { GSH content } \\
\text { lipid peroxidation }\end{array}$ & {$[131,132]$} \\
\hline 98 & $\begin{array}{c}\text { Laurencia dendroidea } \\
\text { (Rhodophyta, Florideophyceae, Ceramiales) }\end{array}$ & $\begin{array}{l}\text { DPPH scavenging: } 30.3 \% \text { at } 2.12 \mathrm{mM} \\
\qquad \mathrm{H}_{2} \mathrm{O}_{2} \text { scavenging }\end{array}$ & [133] \\
\hline 99 & $\begin{array}{c}\text { L. dendroidea } \\
\text { (Rhodophyta, Florideophyceae, Ceramiales) }\end{array}$ & $\begin{array}{l}\text { DPPH scavenging: } 27.5 \% \text { at } 2.12 \mathrm{mM} \\
\mathrm{H}_{2} \mathrm{O}_{2} \text { scavenging }\end{array}$ & [133] \\
\hline 100 & $\begin{array}{c}\text { S. wightii } \\
\text { (Ochrophyta, Phaeophyceae, Fucales) }\end{array}$ & $\begin{array}{l}\text { ABTS }{ }^{+} \text {scavenging } \mathrm{IC}_{50}=1.18 \pm 0.07 \mathrm{mM} \\
\text { DPPH scavenging: } \mathrm{IC}_{50}=1.08 \pm 0.07 \mathrm{mM}\end{array}$ & [134] \\
\hline 101 & $\begin{array}{c}\text { S. wightii } \\
\text { (Ochrophyta, Phaeophyceae, Fucales) }\end{array}$ & $\begin{array}{l}\text { ABTS }{ }^{+} \text {scavenging: } \mathrm{IC}_{50}=0.72 \pm 0.09 \mathrm{mM} \\
\text { DPPH scavenging: } \mathrm{IC}_{50}=0.75 \pm 0.03 \mathrm{mM}\end{array}$ & [134] \\
\hline 102 & $\begin{array}{c}\text { Cystoseira trinodis } \\
\text { (Ochrophyta, Phaeophyceae, Fucales) }\end{array}$ & $\begin{array}{c}\text { ABTS }^{+} \text {scavenging: } 24.19 \pm 1.15 \% \text { inhibition at } \\
2 \mathrm{mM}\end{array}$ & [135] \\
\hline 103 & $\begin{array}{c}\text { C. trinodis } \\
\text { (Ochrophyta, Phaeophyceae, Fucales) }\end{array}$ & $\begin{array}{c}\text { ABTS }^{+} \text {scavenging: } 27.50 \pm 1.30 \% \text { inhibition at } \\
2 \mathrm{mM}\end{array}$ & [135] \\
\hline 104 & $\begin{array}{c}\text { C. trinodis } \\
\text { (Ochrophyta, Phaeophyceae, Fucales) } \\
\text { E. stolonifera, E. bicyclis } \\
\text { (Ochrophyta, Phaeophyceae, Laminariales) }\end{array}$ & $\begin{array}{c}\text { ABTS }^{+} \text {scavenging: } 24.05 \pm 2.38 \% \text { inhibition at } \\
22 \mathrm{mM} \\
\text { intracellular ROS generation (DCFH-DA) } \\
\text { intracellular GSH levels in t-BHP- and } \\
\text { tacrine-treated HepG2 cells } \\
\text { t-BHP- and tacrine-induced oxidative stress in } \\
\text { HepG2 cells }\end{array}$ & {$[135,136]$} \\
\hline 105 & $\begin{array}{c}\text { C. trinodis } \\
\text { (Ochrophyta, Phaeophyceae, Fucales) }\end{array}$ & $\begin{aligned} & \text { ABTS }^{+} \text {scavenging: } 26.37 \pm 0.20 \% \text { inhibition at } \\
& 2 \mathrm{mM}\end{aligned}$ & [135] \\
\hline 106 & $\begin{array}{c}\text { C. trinodis } \\
\text { (Ochrophyta, Phaeophyceae, Fucales) }\end{array}$ & $\begin{array}{c}\text { ABTS }^{+} \text {scavenging: } 20.41 \pm 0.13 \% \text { inhibition at } \\
2 \mathrm{mM}\end{array}$ & [135] \\
\hline 107 & $\begin{array}{c}\text { Caulerpa racemosa } \\
\text { (Chlorophyta, Ulvophyceae, Bryopsidales) }\end{array}$ & $\begin{array}{l}\text { Alkyl scavenging: } \mathrm{IC}_{50}=0.66 \pm 0.05 \mathrm{mM} \\
\text { OH scavenging: } \mathrm{IC}_{50}=0.29 \pm 0.05 \mathrm{mM}\end{array}$ & [137] \\
\hline
\end{tabular}


Table 3. Cont.

\begin{tabular}{|c|c|c|c|}
\hline Compound & Isolation Source & Assay/Activity & Reference \\
\hline 108 & $\begin{array}{c}\text { S. wightii } \\
\text { (Ochrophyta, Phaeophyceae, Fucales) }\end{array}$ & $\begin{array}{l}\text { ABTS }^{+} \text {scavenging } \mathrm{IC}_{50}=0.37 \pm 0.02 \mathrm{mM} \\
\text { DPPH scavenging: } \mathrm{IC}_{50}=0.31 \pm 0.02 \mathrm{mM}\end{array}$ & [134] \\
\hline 109 & $\begin{array}{c}\text { S. wightii } \\
\text { (Ochrophyta, Phaeophyceae, Fucales) }\end{array}$ & $\begin{array}{l}\text { ABTS }{ }^{+} \text {scavenging: } \mathrm{IC}_{50}=0.37 \pm 0.02 \mathrm{mM} \\
\text { DPPH scavenging: } \mathrm{IC}_{50}=0.34 \pm 0.06 \mathrm{mM}\end{array}$ & [134] \\
\hline 110 & $\begin{array}{c}\text { Gracilaria salicornia } \\
\text { (Rhodophyta, Florideophyceae, Gracilariales) }\end{array}$ & $\begin{array}{l}\text { ABTS }{ }^{+} \text {scavenging: } \mathrm{IC}_{50}=1.09 \mathrm{mM} \\
\text { DPPH scavenging: } \mathrm{IC}_{50}=1.33 \mathrm{mM}\end{array}$ & [138] \\
\hline 111 & $\begin{array}{c}\text { G. salicornia } \\
\text { (Rhodophyta, Florideophyceae, Gracilariales) }\end{array}$ & $\begin{array}{l}\text { ABTS }+ \text { scavenging: } \mathrm{IC}_{50}=1.24 \mathrm{mM} \\
\text { DPPH scavenging: } \mathrm{IC}_{50}=1.56 \mathrm{mM}\end{array}$ & [138] \\
\hline 112 & $\begin{array}{l}\text { from plants and microalgae, but also from } \\
\text { macroalgae }\end{array}$ & $\begin{array}{c}\text { enzyme activity (CAT, SOD, GPx and GSH } \\
\text { reductase) } \\
\text { GSH and TBARS levels in hepatic tissue of } \\
\text { lycopene-treated rats }\end{array}$ & [139] \\
\hline
\end{tabular}

113 from plants and microalgae, but also from macroalgae

intracellular ROS generation in LPS-stimulated

RAW 264.7 macrophages

113

114

from plants and microalgae, but also from macroalgae
LPS- and IFN- $\gamma$-induced NO generation in RAW 264.7 macrophages

TPA-induced $\mathrm{O}_{2}{ }^{-}$generation in differentiated human promyelocytic HL-60 cells

LPS- and IFN- $\gamma$-induced NO generation in RAW 264.7 macrophages

TPA-induced $\mathrm{O}_{2}{ }^{-}$generation in differentiated human promyelocytic HL-60 cells

LPS- and IFN- $\gamma$-induced NO generation in RAW

$$
264.7 \text { macrophages }
$$

TPA-induced $\mathrm{O}_{2}{ }^{-}$generation in differentiated human promyelocytic HL-60 cells

$$
\text { radical scavenging }
$$

enzyme (SOD2, CAT, and GPx1) regulation in irradiated cells

intracellular ROS generation (DCFH-DA) in acetaldehyde-treated $\mathrm{SH}-\mathrm{SY} 5 Y$ cells

LPS- and IFN- $\gamma$-induced NO generation in RAW 264.7 macrophages

Nrf2/HO-1 antioxidant pathway

Nrf2 dissociation and nuclear translocation $\mathrm{Nrf} 2$ expression regulation in irradiated cells

Nrf2-regulated enzymes expression (HO-1, NQO-1, and GST- $\alpha 1$ )

PI3K/Akt and ERK signaling pathway regulation

ROS-induced oxidative stress in a rat deep-burn model

regulation of free radical production

(XO/reduced form of Nox)

Sp1/NR1 signaling pathway regulation TPA-induced $\mathrm{O}_{2}{ }^{-}$generation in differentiated human promyelocytic HL-60 cells Akt/CREB and p38 kinase/MAPK signaling pathway in acetaldehyde-treated $\mathrm{SH}-\mathrm{SY} 5 \mathrm{Y}$ cells 
Table 3. Cont.

\begin{tabular}{|c|c|c|c|}
\hline Compound & Isolation Source & Assay/Activity & Reference \\
\hline 118 & from various species of Ochrophyta & 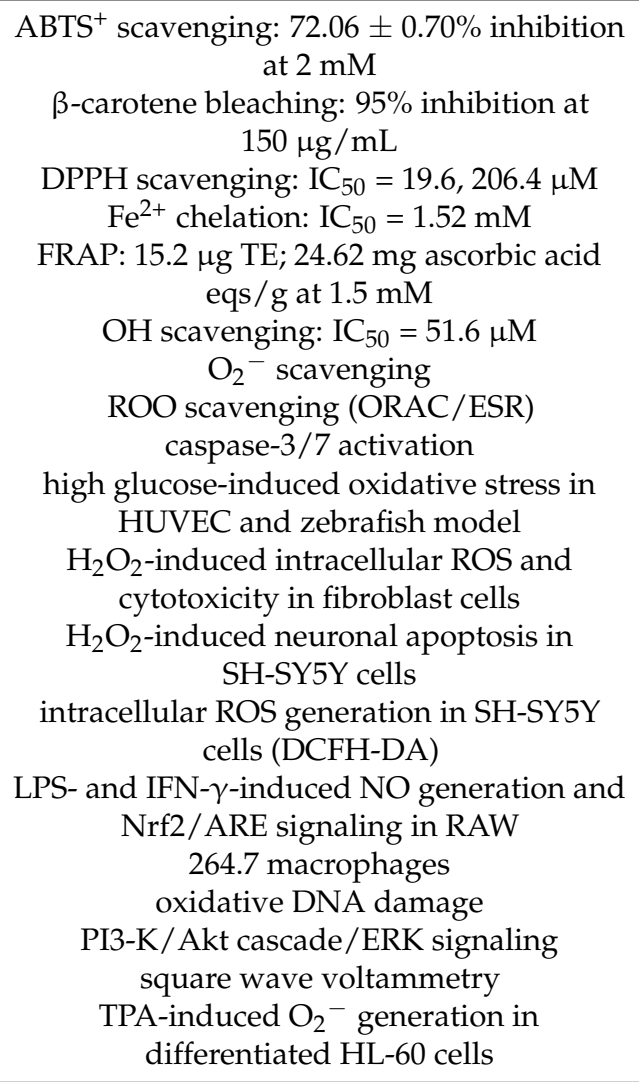 & {$[142,153-162]$} \\
\hline 119 & $\begin{array}{c}\text { Laminaria japonica } \\
\text { (Ochrophyta, Phaeophyceae, Laminariales) }\end{array}$ & $\begin{array}{l}\mathrm{ABTS}^{+} \text {scavenging } \\
\text { DPPH scavenging } \\
\mathrm{OH} \text { scavenging } \\
\mathrm{O}_{2}^{-} \text {scavenging }\end{array}$ & [162] \\
\hline 120 & $\begin{array}{c}\text { L. japonica } \\
\text { (Ochrophyta, Phaeophyceae, Laminariales) }\end{array}$ & $\begin{array}{l}\mathrm{ABTS}^{+} \text {scavenging } \\
\text { DPPH scavenging } \\
\mathrm{OH} \text { scavenging } \\
\mathrm{O}_{2}^{-} \text {scavenging }\end{array}$ & [162] \\
\hline 121 & $\begin{array}{c}\text { L. japonica } \\
\text { (Ochrophyta, Phaeophyceae, Laminariales) }\end{array}$ & $\begin{array}{c}\mathrm{ABTS}^{+} \text {scavenging } \\
\text { DPPH scavenging } \\
\mathrm{OH} \text { scavenging } \\
\mathrm{O}_{2}^{-} \text {scavenging }\end{array}$ & [162] \\
\hline 122 & $\begin{array}{l}\text { from plants and microalgae, but also from } \\
\text { macroalgae }\end{array}$ & $\begin{array}{l}\text { ABTS }{ }^{+} \text {scavenging: } \mathrm{IC}_{50}=25.4 \mu \mathrm{M} \\
\text { DPPH scavenging: } \mathrm{IC}_{50}=68.9 \mu \mathrm{M}\end{array}$ & [163] \\
\hline 123 & $\begin{array}{c}\text { Undariopsis peterseniana } \\
\text { (Ochrophyta, Phaeophyceae, Laminariales) }\end{array}$ & oxidative stress-mediated apoptosis & [164] \\
\hline 124 & $\begin{array}{c}\text { Sargassum horneri } \\
\text { (Ochrophyta, Phaeophyceae, Fucales) }\end{array}$ & $\begin{array}{c}\text { alkyl scavenging (ESR): } \mathrm{IC}_{50}: 0.22 \pm 0.02 \mathrm{mM} \\
\text { AAPH-induced intracellular ROS in Vero } \\
\text { cells } \\
\text { AAPH-induced lipid peroxidation in } \\
\text { zebrafish models in vivo } \\
\text { NF-kB, MAPK and oxidative stress } \\
\text { regulation in RAW } 264.7 \text { macrophages } \\
\text { Nrf2/HO-1 pathways regulation }\end{array}$ & {$[165,166]$} \\
\hline
\end{tabular}

AAPH: 2,2'-azobis(2-amidinopropane) dihydrochloride; $\mathrm{ABTS}^{+}:$2,2'-azino-bis (3-ethyl benzothiazoline-6-sulfonic acid) diammonium salt; Akt: protein kinase B; ARE: antioxidant response element; CAT: catalase; DCFH-DA: cell-based $2^{\prime}, 7^{\prime}$-dichlorodihydrofluorescein diacetate 
antioxidant assay; DPPH: 1,1-diphenyl-2-picrylhydrazyl free radical; ESR: electron spin resonance; FRAP: ferric reducing antioxidant power; GSH: glutathione; GPx: glutathione peroxidase; HO-1: heme oxygenase- $1 ; \mathrm{H}_{2} \mathrm{O}_{2}$ : hydrogen peroxide; HUVEC: human umbilical vein endothelial cells; $\mathrm{OH}$ : hydroxyl; $\mathrm{IC}_{50}$ : half maximal inhibitory concentration; IFN- $\gamma$ : interferon $\gamma$; LPS: lipopolysaccharide; MAPK: mitogenactivated protein kinase; NADPH: nicotinamide adenine dinucleotide phosphate; NF- $\mathrm{kB}$ : nuclear factor kappa-light-chain-enhancer of activated B cells, NO.: nitric oxide; Nox: NADPH oxidase; Nrf2: nuclear factor erythroid 2-related factor 2; $\mathrm{O}_{2}{ }^{-}$: superoxide anion; ORAC: oxygen radical absorbance capacity; PI3-K: phosphatidylinositol 3-kinase; ROS: reactive oxygen species; SH-SY5Y: human dopaminergic neuronal cell line; SOD: superoxide dismutase; TBARS: thiobarbituric acid reactive substances; t-BHP: tert-butyl hydroperoxide; TE: trolox equivalents; TPA: 12-O-tetradecanoylphorbol 13-acetate; XO: xanthine oxidase.<smiles>CC(Cl)(/C=C/Br)C(Cl)/C=C/C(=C/Cl)CCl</smiles><smiles>[R]C1([R])CC(C)=C(CCC(C)C)C(C)(C)C1</smiles>

$93 \mathrm{R}_{1}=\mathrm{R}_{2}=\mathrm{H}$

$94 \mathrm{R}_{1}=\mathrm{OH}, \mathrm{R}_{2}=\mathrm{H}$

$95 \mathrm{R}_{1}=\mathrm{H}, \mathrm{R}_{2}=\mathrm{OH}$

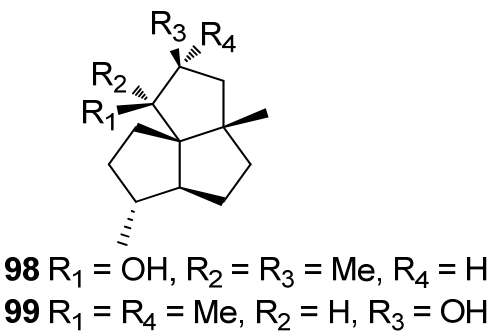<smiles>CC(C)=CCC[C@H]1C=C(C)[C@@H](O)CC1(C)C</smiles>

91<smiles>CCC(=O)CCC1(C)C(C)=CC(=O)CC1(C)C</smiles>

96<smiles>C=CC(C)/C=C/C1C(=C)CCC2C(C)(C)CC(O)CC12C</smiles><smiles>CC1=CC(=O)CC(C)(C)C1(O)/C=C/C(C)=C\C(=O)O</smiles>

92<smiles>Cc1cc2c(cc1Br)C1(C)CC[C@H](C)C1(C)O2</smiles>

97<smiles>C=C[C@](C)(O)/C=C/[C@H]1C(=C)C[C@H](O)[C@H](O)C1(C)CC(O)CC</smiles>

Figure 9. Chemical structures of compounds 90-101.

Compared to phenolic compounds, as presented in Table 2, it is evident that terpenoids are less active, since their $\mathrm{IC}_{50}$ values in the DPPH radical scavenging assay are mostly within the $\mathrm{mM}$ range. The most active compounds reported are the halogenated monoterpene (1E,3R,4S,5E,7Z)-1-bromo-3,4,8-trichloro-7-(dichloro-methyl)-3-methyl- octa1,5,7-triene (90), isolated from the red alga Plocamium sp., and the carotenoids fucoxanthin (118) and violaxanthin (122), isolated from various macroalgae, with $\mathrm{IC}_{50}$ values of 50.0, 19.6 , and $68.9 \mu \mathrm{M}$, respectively $[128,159,163]$.

Alarif et al. (2015) isolated a series of C-29 steroids (102-106), along with fucoxanthin (118), from the brown alga Cystoseira trinodis and all compounds were evaluated for their antioxidant activity [135]. Steroids 102-106 showed moderate antioxidant activity (20.4 to $27.5 \%)$ in the ABST assay, while compound 118 exhibited significant levels of activity (72.1\%).

Fucosterol (104), frequently isolated from brown algae, was confirmed to exert antioxidant activity on hepatic cells via an increase in the hepatic levels of GSH and a decrease in ROS production, therefore preventing hepatic damage and the resultant increase in alanine transaminase and aspartate transaminase activities [136]. Hence, fucosterol is considered an effective hepatoprotective agent that could be useful for preventive therapies against oxidative stress-related hepatotoxicity.

Moreover, the abeo-oleanenes $\mathbf{1 1 0}$ and $\mathbf{1 1 1}$ were isolated from the red alga Gracilaria salicornia and their antioxidant activity was evaluated employing the DPPH and $\mathrm{ABTS}^{+}$ radical scavenging assays [138]. Compound 110 exhibited higher radical scavenging activities $\left(\mathrm{DPPH} \mathrm{IC} \mathrm{IC}_{50}=1.33 \mathrm{mM} ; \mathrm{ABTS}^{+} \mathrm{IC}_{50}=1.09 \mathrm{mM}\right)$, when compared to those 
displayed by compound $111\left(\mathrm{DPPH} \mathrm{IC}_{50}=1.56 \mathrm{mM}\right.$; $\left.\mathrm{ABTS}^{+} \mathrm{IC}_{50}=1.24 \mathrm{mM}\right)$ and $\alpha-$ tocopherol that was used as positive control $\left(\mathrm{DPPH} \mathrm{IC} 50=1.46 \mathrm{mM} ; \mathrm{ABTS}^{+} \mathrm{IC}_{50}=1.72 \mathrm{mM}\right.$ ).<smiles>CCC(CCC(C)[C@H]1CCC2C3CC=C4C[C@@H](O)CC[C@]4(C)C3CC[C@@]21C)C(C)C</smiles>

102<smiles>C=CC(CCC(C)[C@H]1CCC2C3CC=C4C[C@@H](O)CC[C@]4(C)C3CC[C@]21C)C(C)C</smiles>

105<smiles>[R][R4]=O</smiles>

$104 \mathrm{R}_{1}=\mathrm{H}, \mathrm{R}_{2}=\mathrm{OH}$<smiles>CC(C)/C(=C/CO)CC[C@H](C)[C@H]1CCC2C3CC=C4C[C@@H](O)CC[C@]4(C)C3CC[C@]21C</smiles>

106<smiles>CC(C)=CCC/C(C)=C/CC/C(C)=C/CC/C=C(\C)CC/C=C(\C)CCC=C(C)C</smiles>

107

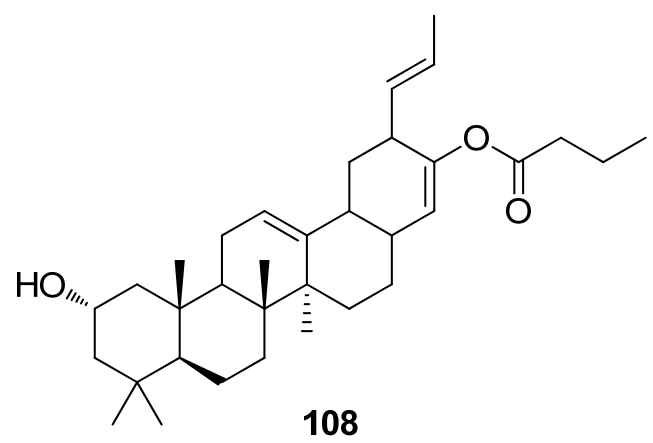<smiles>C/C=C/CCC(=O)OC1=CC2CC[C@]3(C)C(=CCC4[C@@]5(C)C[C@@H](O)CC(C)(C)[C@@H]5CC[C@]43C)C2CC1CCC</smiles>

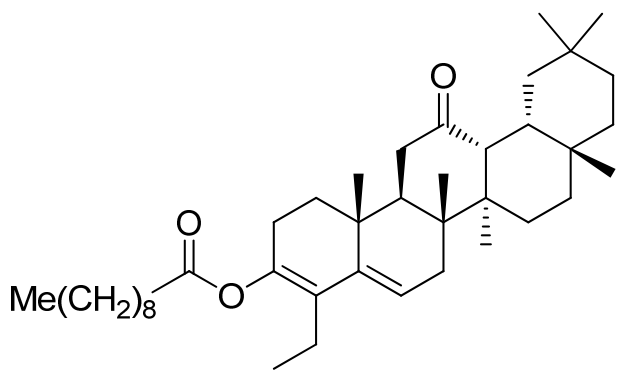

110<smiles>C/C=C/CCCC(=O)OCC12C=CC(CC)C[C@H]1[C@@H]1C(=O)C[C@H]3[C@@H](CC[C@@H]4[C@H](CC)CC=C[C@]34C)[C@@]1(CC)C=C2</smiles>

111

Figure 10. Chemical structures of compounds 102-111.

Among terpenoids, carotenoids, a family of lipophilic pigments synthesized by plants, algae, fungi, and microorganisms, but not animals, exhibit high levels of antioxidant activity. In red, brown, and green algae, carotenoids play a key role in their protection against photo-oxidative processes [6]. Their antioxidant action is based on their singlet oxygen quenching properties and their free radicals scavenging ability, which mainly 
depends on the number of conjugated double bonds, the nature of substituents and the end groups of the carotenoids [6].

In marine macroalgae, $\beta$-carotene (113), lutein (114), zeaxanthin (115), astaxanthin (116), neoxanthin (117), fucoxanthin (118), and violaxanthin (122) are known to be among the major carotenoids encountered [167]. Astaxanthin (116) acts as a safeguard against oxidative damage through various mechanisms, such as singlet oxygen quenching, radical scavenging, inhibition of lipid peroxidation, and regulation of gene expression related to oxidative stress [144,148,168-171]. The exact mechanisms of action of astaxanthin have been extensively studied, since it has been proven to confer protective effects against neurological diseases, as well as in treating and preventing skin diseases [171-173].

Specifically, astaxanthin (116) activates the phosphatidylinositol 3-kinase (PI3K)/Akt and ERK signaling pathways, and thus facilitates the dissociation and nuclear translocation of Nrf2, which leads to upregulation of the expression of Nrf2-regulated enzymes (e.g., HO-1, NQO-1, and GST- $\alpha 1$ ) [147]. Astaxanthin (116) inhibits the production of intracellular ROS by negatively regulating the Sp1/NR1 signaling pathway $[149,150]$ and modulating the expression of oxidative stress-responsive enzymes, such as HO-1, which is a marker of oxidative stress and a regulatory mechanism involved in cell adaptation against oxidative damage [143]. In addition, astaxanthin activates the Nrf2/HO-1 antioxidant pathway by generating small amounts of ROS $[145,146]$. In agreement with these studies, Xue et al. (2017) observed that astaxanthin upregulated Nrf2 expression, as well as Nrf2-targeted proteins HO-1 and antioxidative enzymes SOD2, CAT, and GPx1 in irradiated cells [151]. Thus, astaxanthin (116) exerts noteworthy antioxidant activities via both direct radical scavenging, and activation of the cellular antioxidant defense system through modulation of the Nrf2 pathway. Furthermore, a recent study in a rat deep-burn model demonstrated astaxanthin's protective role in early burn-wound progression by controlling ROS-induced oxidative stress. In that case, the regulation of free radical production is due to the influence of xanthine oxidase and the reduced form of nicotinamide adenine dinucleotide phosphate oxidase, both contributing to the generation of ROS [144].<smiles>CC(C)=CCC/C(C)=C/C=C/C(C)=C/C=C/C(C)=C/C=C/C=C(C)/C=C/C=C(C)/C=C/C=C(\C)CCC=C(C)C</smiles><smiles>CC1=C(/C=C/C(C)=C/C=C/C(C)=C/C=C/C=C(C)/C=C/C=C(C)/C=C/C2=C(C)CCCC2(C)C)C(C)(C)CCC1</smiles><smiles>CC1=C[C@@H](O)CC(C)(C)[C@H]1/C=C/C(C)=C/C=C/C(C)=C/C=C/C=C(C)/C=C/C=C(C)/C=C/C1=C(C)C[C@@H](O)CC1(C)C</smiles>

114<smiles>CC1=C(/C=C/C(C)=C/C=C/C(C)=C/C=C/C=C(C)/C=C/C=C(C)/C=C/C2=C(C)C[C@@H](O)CC2(C)C)C(C)(C)C[C@H](O)C1</smiles>

Figure 11. Chemical structures of compounds 112-115. 
<smiles>[Z17]C(/C=C/C=C(C)/C=C/C=C(C)/C=C/C1=C(C)C(=O)[C@@H](O)CC1(C)C)=C\C=C\C1=C(C)C(=O)[C@@H](O)CC1(C)C</smiles><smiles>CC(=O)OC1CC(C)(C)C(=C=C=C(C)C=CC(C)=CC=CC=C(C)C=CC=C(C)C(=O)C[C@@]23O[C@]2(C)CC(O)CC3(C)C)C(C)(O)C1</smiles><smiles>C=C(C)/C=C/C=C(\C)CCCC(C)(C)C</smiles><smiles>CC(=O)OC1CC(C)(C)C(=C=C=C(C)C=CC=C(C)C=CC=CC=C(C)C=CC=C(C)C(=O)C[C@@]23O[C@]2(C)CC(O)CC3(C)C)C(C)(C)C1</smiles>

120<smiles>C=C/C=C(C)/C=C/C=C/C(C)=C/C=C1C(C)(C)C[C@@H](OC(C)=O)CC1(C)O</smiles><smiles>C/C=C(\C)C=CC12OC1(C)C(C)(C)C[C@@H](O)CC2(C)O</smiles>

123<smiles>C=C(C)/C=C/C=C(C)/C=C/C1(C)C(C)(C)CC(O)CC1(C)C</smiles>

122

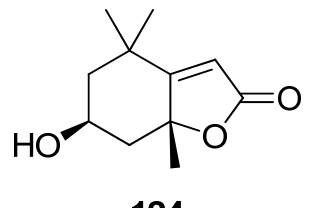

124

Figure 12. Chemical structures of compounds 116-124. 
Fucoxanthin (118), often isolated from brown algae, is an oxo-carotenoid with an allenic carbon moiety and a 5,6-monoepoxide in its structure, acknowledged as an efficient quencher of singlet oxygen in photooxidation [174-176]. The antioxidant activity of fucoxanthin (118) is mediated through various mechanisms, such as singlet oxygen quenching, radical scavenging, and inhibition of lipid peroxidation. Fucoxanthin (118) has been shown to exert the best in vitro bioactivities among carotenoids in inhibiting overexpression of vascular endothelial growth factor, resisting senescence, improving phagocytic function, and clearing intracellular ROS in retinal pigment epithelium cells, protecting the retina against photoinduced damage [156].

The study of Taira et al. (2017) demonstrated that fucoxanthin (118), through the Nrf2 activation, exerts either cytoprotective activity or induction of apoptosis, depending on the concentrations employed [153]. At a low concentration range $(1-4 \mu \mathrm{M})$, fucoxanthin provides a cytoprotective effect due to its antioxidant activity, as exerted by its peroxyl radical scavenging capacity, involving the antioxidant $\mathrm{HO}-1$ protein expression increase through the activation of the Nrf2/ARE pathway. On the other hand, high concentration $(>10 \mu \mathrm{M})$ treatment of cells induces apoptosis with caspase $-3 / 7$ activation during the suppression of anti-apoptotic proteins, such as Bcl-xL and pAkt.

Besides, the cytoprotective effect of fucoxanthin (118) has been investigated against $\mathrm{H}_{2} \mathrm{O}_{2}$-induced cell damage [154,158]. It was shown that fucoxanthin effectively inhibited intracellular ROS formation, DNA damage, and apoptosis induced by $\mathrm{H}_{2} \mathrm{O}_{2}$. Finally, the protective effect of fucoxanthin was investigated against UVB-induced cell injury in human fibroblasts and showed significant decrease in intracellular ROS formation and increase in cell survival rate in a dose-dependent manner [155].

Comparative studies of the radical scavenging efficiency of fucoxanthin (118) and its stereoisomers (119-121) isolated from Laminaria japonica have also been conducted [162]. All three stereoisomers had stronger hydroxyl radical scavenging activities than $\alpha$-tocopherol but showed weaker scavenging activities toward DPPH and superoxide radical, while their radical scavenging activities were not remarkably different, indicating that the differences in the geometry of the double bonds had very little effect on their activity.

Recently, the monoterpenoid (-)-loliolide (124) was proven to effectively reduce 2,2'-azobis(2-amidinopropane) dihydrochloride (AAPH)-induced ROS, cell death, and lipid peroxidation in Vero cells and zebrafish embryos in a dose-dependent manner [165]. Moreover, a study conducted by Jayawardena et al. (2019) elaborated the anti-inflammatory effect of Sargassum horneri ethanolic extract containing (-)-loliolide on LPS-stimulated RAW 264.7 macrophages via suppression of NF- $\mathrm{KB}$ and MAPK and reduction of oxidative stress through the Nrf2/HO-1 pathway [166].

\section{Meroterpenoids}

Meroterpenoids are natural products of mixed biosynthesis containing a terpenoid part that exhibit a variety of biological activities. Metabolites belonging to this class that display antioxidant activity have been isolated from various macroalgae (Table 4, Figures 13-19), the majority of which belong to the phylum Ochrophyta, and especially to the genera Cystoseira and Sargassum.

Table 4. Meroterpenoids from macroalgae with antioxidant activity.

\begin{tabular}{|c|c|c|c|}
\hline Compound & Isolation Source & Assay/Activity & Reference \\
\hline 125 & $\begin{array}{c}\text { Cymopolia barbata } \\
\text { (Chlorophyta, Ulvophyceae, Dasycladales) }\end{array}$ & $\begin{array}{c}\text { DPPH scavenging: strong } \\
\text { exogenous ROS scavenging in TPA-treated } \\
\text { HL-60 cells (DCFH-DA): } \mathrm{IC}_{50}=4.0 \mu \mathrm{M}\end{array}$ & [91] \\
\hline 126 & $\begin{array}{c}\text { C. barbata } \\
\text { (Chlorophyta, Ulvophyceae, Dasycladales) }\end{array}$ & $\begin{array}{c}\text { DPPH scavenging: strong } \\
\text { exogenous ROS scavenging in TPA-treated } \\
\text { HL-60 cells (DCFH-DA): IC } 50>14.6 \mu \mathrm{M}\end{array}$ & [91] \\
\hline
\end{tabular}


Table 4. Cont.

\begin{tabular}{|c|c|c|c|}
\hline Compound & Isolation Source & Assay/Activity & Reference \\
\hline 127 & $\begin{array}{c}\text { Cystoseira crinita } \\
\text { (Ochrophyta, Phaeophyceae, Fucales) }\end{array}$ & $\begin{array}{c}\text { ABTS }^{+} \text {scavenging } \\
\text { DPPH scavenging: } 94.1 \% \text { at } 230 \mu \mathrm{M} \\
\mathrm{O}_{2}{ }^{-} \text {generation (PCL assay) } \\
\text { TBARS assay: } 66.8 \% \text { inhibition at } 164 \mu \mathrm{M}\end{array}$ & [177] \\
\hline 128 & $\begin{array}{c}\text { C. crinita } \\
\text { (Ochrophyta, Phaeophyceae, Fucales) }\end{array}$ & $\begin{array}{c}\text { ABTS }^{+} \text {scavenging } \\
\text { DPPH scavenging activity: } 92.5 \% \text { at } 230 \mu \mathrm{M} \\
\mathrm{O}_{2}^{-} \text {generation (PCL assay) } \\
\text { TBARS assay: } 66.5 \% \text { inhibition at } 164 \mu \mathrm{M}\end{array}$ & [177] \\
\hline 129 & $\begin{array}{c}\text { Cystoseira usneoides } \\
\text { (Ochrophyta, Phaeophyceae, Fucales) }\end{array}$ & $\mathrm{ABTS}^{+}$scavenging: $\mathrm{IC}_{50}=33.3 \pm 2.3 \mu \mathrm{M} ; 0.78 \mathrm{TE}$ & [178] \\
\hline 130 & $\begin{array}{c}\text { C. usneoides } \\
\text { (Ochrophyta, Phaeophyceae, Fucales) }\end{array}$ & ABTS $^{+}$scavenging: $\mathrm{IC}_{50}=51.6 \pm 4.8 \mu \mathrm{M} ; 0.50 \mathrm{TE}$ & [178] \\
\hline 131 & $\begin{array}{c}\text { C. usneoides } \\
\text { (Ochrophyta, Phaeophyceae, Fucales) }\end{array}$ & ABTS $^{+}$scavenging: $\mathrm{IC}_{50}=44.7 \pm 1.1 \mu \mathrm{M} ; 0.58 \mathrm{TE}$ & [178] \\
\hline 132 & $\begin{array}{c}\text { C. usneoides } \\
\text { (Ochrophyta, Phaeophyceae, Fucales) }\end{array}$ & ABTS $^{+}$scavenging: $\mathrm{IC}_{50}=55.9 \pm 9.9 \mu \mathrm{M} ; 0.46 \mathrm{TE}$ & [178] \\
\hline 133 & $\begin{array}{c}\text { Dictyopteris undulata } \\
\text { (Ochrophyta, Phaeophyceae, Dictyotales) }\end{array}$ & DPPH scavenging: $\mathrm{IC}_{50}=71 \mu \mathrm{M}$ & [179] \\
\hline 134 & $\begin{array}{c}\text { D. undulata } \\
\text { (Ochrophyta, Phaeophyceae, Dictyotales) }\end{array}$ & $\begin{array}{c}\text { expression of phase-2 enzymes (i.e., NQO1, GSH } \\
\text { S-transferase, HO-1 and PRDX4) } \\
\text { Nrf2/ ARE signaling pathway } \\
\text { oxidative stress in HT22 hippocampal neuronal } \\
\text { cells }\end{array}$ & [180] \\
\hline 135 & $\begin{array}{c}\text { D. undulata } \\
\text { (Ochrophyta, Phaeophyceae, Dictyotales) }\end{array}$ & DPPH scavenging: $\mathrm{IC}_{50}=121 \mu \mathrm{M}$ & [179] \\
\hline 136 & $\begin{array}{c}\text { G. salicornia } \\
\text { (Rhodophyta, Florideophyceae, Gracilariales) }\end{array}$ & $\begin{array}{l}\text { ABTS }{ }^{+} \text {scavenging: } \mathrm{IC}_{50}=1.88 \pm 0.02 \mathrm{mM} \\
\text { DPPH scavenging: } \mathrm{IC}_{50}=1.51 \pm 0.01 \mathrm{mM}\end{array}$ & [181] \\
\hline 137 & $\begin{array}{c}\text { G. salicornia } \\
\text { (Rhodophyta, Florideophyceae, Gracilariales) }\end{array}$ & $\begin{array}{l}\text { ABTS }{ }^{+} \text {scavenging: } \mathrm{IC}_{50}=1.96 \pm 0.01 \mathrm{mM} \\
\text { DPPH scavenging: } \mathrm{IC}_{50}=1.85 \pm 0.02 \mathrm{mM}\end{array}$ & [181] \\
\hline 138 & $\begin{array}{c}\text { G. salicornia } \\
\text { (Rhodophyta, Florideophyceae, Gracilariales) }\end{array}$ & $\begin{array}{l}\text { ABTS }{ }^{+} \text {scavenging: } \mathrm{IC}_{50}=1.57 \pm 0.02 \mathrm{mM} \\
\text { DPPH scavenging: } \mathrm{IC}_{50}=1.33 \pm 0.01 \mathrm{mM}\end{array}$ & [181] \\
\hline 139 & $\begin{array}{c}\text { D. undulata } \\
\text { (Ochrophyta, Phaeophyceae, Dictyotales) }\end{array}$ & DPPH scavenging: $\mathrm{IC}_{50}=145 \mu \mathrm{M}$ & [179] \\
\hline 140 & $\begin{array}{c}\text { G. salicornia } \\
\text { (Rhodophyta, Florideophyceae, Gracilariales) }\end{array}$ & $\begin{array}{l}\text { ABTS }{ }^{+} \text {scavenging: } \mathrm{IC}_{50}=1.50 \mathrm{mM} \\
\text { DPPH scavenging: } \mathrm{IC}_{50}=1.40 \mathrm{mM}\end{array}$ & [182] \\
\hline 141 & $\begin{array}{c}\text { G. salicornia } \\
\text { (Rhodophyta, Florideophyceae, Gracilariales) }\end{array}$ & $\begin{array}{l}\text { ABTS }+ \text { scavenging: } \mathrm{IC}_{50}=1.33 \mathrm{mM} \\
\text { DPPH scavenging: } \mathrm{IC}_{50}=1.17 \mathrm{mM}\end{array}$ & [182] \\
\hline 142 & $\begin{array}{c}\text { S. micracanthum } \\
\text { (Ochrophyta, Phaeophyceae, Fucales) }\end{array}$ & $\begin{array}{c}\text { DPPH scavenging: } \mathrm{IC}_{50}=25.5 \mu \mathrm{M} \\
\text { lipid peroxidation in rat liver: } \mathrm{IC}_{50}=0.26 \mu \mathrm{M}\end{array}$ & [183] \\
\hline 143 & $\begin{array}{c}\text { S. micracanthum } \\
\text { (Ochrophyta, Phaeophyceae, Fucales) }\end{array}$ & $\begin{array}{c}\text { DPPH scavenging: } 3.0 \% \text { at } 0.23 \mathrm{mM} \\
\text { lipid peroxidation in rat liver: } \mathrm{IC}_{50}=2.22 \mu \mathrm{M}\end{array}$ & [184] \\
\hline 144 & $\begin{array}{l}\text { Cystoseira abies-marina } \\
\text { (Ochrophyta, Phaeophyceae, Fucales) }\end{array}$ & DPPH scavenging: $29 \%$ at $1.06 \mathrm{mM}$ & [185] \\
\hline 145 & $\begin{array}{c}\text { C. abies-marina } \\
\text { (Ochrophyta, Phaeophyceae, Fucales) }\end{array}$ & DPPH scavenging: $30 \%$ at $1.02 \mathrm{mM}$ & [185] \\
\hline
\end{tabular}


Table 4. Cont.

\begin{tabular}{|c|c|c|c|}
\hline Compound & Isolation Source & Assay/Activity & Reference \\
\hline 146 & $\begin{array}{c}\text { C. crinita } \\
\text { (Ochrophyta, Phaeophyceae, Fucales) }\end{array}$ & $\begin{array}{l}\text { ABTS }^{+} \text {scavenging } \\
\text { DPPH scavenging: } 94.4 \% \text { at } 230 \mu \mathrm{M} \\
\mathrm{O}_{2}^{-} \text {radical generation (PCL assay) } \\
\text { TBARS: } 70.8 \% \text { inhibition at } 164 \mu \mathrm{M}\end{array}$ & [177] \\
\hline 147 & $\begin{array}{c}\text { C. crinita } \\
\text { (Ochrophyta, Phaeophyceae, Fucales) }\end{array}$ & $\begin{array}{l}\text { ABTS }^{+} \text {scavenging: TEAC }=0.14 \mathrm{mM} \\
\text { DPPH scavenging: } 95.4 \% \text { at } 230 \mu \mathrm{M} \\
\mathrm{O}_{2}{ }^{-} \text {radical generation }(\mathrm{PCL} \text { assay): } 1.35 \\
\text { TBARS: } 71.8 \% \text { inhibition at } 164 \mu \mathrm{M}\end{array}$ & [177] \\
\hline 148 & $\begin{array}{c}\text { C. crinita } \\
\text { (Ochrophyta, Phaeophyceae, Fucales) }\end{array}$ & $\begin{array}{l}\text { ABTS }^{+} \text {scavenging } \\
\text { DPPH scavenging: } 96.1 \% \text { at } 230 \mu \mathrm{M} \\
\mathrm{O}_{2}{ }^{-} \text {radical generation (PCL assay) } \\
\text { TBARS: } 68.9 \% \text { inhibition at } 164 \mu \mathrm{M}\end{array}$ & [177] \\
\hline 149 & $\begin{array}{c}\text { C. crinita } \\
\text { (Ochrophyta, Phaeophyceae, Fucales) }\end{array}$ & $\begin{array}{l}\text { ABTS }^{+} \text {scavenging } \\
\text { DPPH scavenging: } 95.5 \% \text { at } 230 \mu \mathrm{M} \\
\mathrm{O}_{2}^{-} \text {radical generation (PCL assay) } \\
\text { TBARS: } 70.3 \% \text { inhibition at } 164 \mu \mathrm{M}\end{array}$ & [177] \\
\hline 150 & $\begin{array}{c}\text { C. crinita } \\
\text { (Ochrophyta, Phaeophyceae, Fucales) }\end{array}$ & $\begin{array}{l}\text { ABTS }^{+} \text {scavenging: } \text { TEAC }=0.37 \mathrm{mM} \\
\text { DPPH scavenging: } 95.5 \% \text { at } 230 \mu \mathrm{M} \\
\mathrm{O}_{2}{ }^{-} \text {radical generation }(\mathrm{PCL} \text { assay): } 1.39 \\
\text { TBARS: } 72.2 \% \text { inhibition at } 164 \mu \mathrm{M}\end{array}$ & [177] \\
\hline 151 & $\begin{array}{c}\text { C. crinita } \\
\text { (Ochrophyta, Phaeophyceae, Fucales) }\end{array}$ & $\begin{array}{c}\text { ABTS }^{+} \text {scavenging: TEAC }=0.09 \mathrm{mM} \\
\text { DPPH scavenging: } 95.7 \% \text { at } 230 \mu \mathrm{M} \\
\mathrm{O}_{2}^{-} \text {radical generation (PCL assay): } 0.72 \\
\text { TBARS: } 71.1 \% \text { inhibition at } 164 \mu \mathrm{M}\end{array}$ & [177] \\
\hline 152 & $\begin{array}{c}\text { C. crinita } \\
\text { (Ochrophyta, Phaeophyceae, Fucales) }\end{array}$ & $\begin{array}{l}\text { ABTS }^{+} \text {scavenging: TEAC }=0.09 \mathrm{mM} \\
\text { DPPH scavenging: } 96.4 \% \text { at } 230 \mu \mathrm{M} \\
\mathrm{O}_{2}^{-} \text {radical generation }(\mathrm{PCL} \text { assay): } 0.59 \\
\text { TBARS: } 73.7 \% \text { inhibition at } 164 \mu \mathrm{M}\end{array}$ & [177] \\
\hline 153 & $\begin{array}{c}\text { C. crinita } \\
\text { (Ochrophyta, Phaeophyceae, Fucales) }\end{array}$ & $\begin{array}{l}\text { ABTS }^{+} \text {scavenging: TEAC }=0.09 \mathrm{mM} \\
\text { DPPH scavenging: } 96.7 \% \text { at } 230 \mu \mathrm{M} \\
\mathrm{O}_{2}{ }^{-} \text {radical generation }(\mathrm{PCL} \text { assay): } 0.51 \\
\text { TBARS: } 73.4 \% \text { inhibition at } 164 \mu \mathrm{M}\end{array}$ & [177] \\
\hline 154 & $\begin{array}{c}\text { C. crinita } \\
\text { (Ochrophyta, Phaeophyceae, Fucales) }\end{array}$ & $\begin{array}{c}\text { ABTS }^{+} \text {scavenging: } \text { TEAC }=0.08 \mathrm{mM} \\
\text { DPPH scavenging: } 65.4 \% \text { at } 230 \mu \mathrm{M} \\
\mathrm{O}_{2}{ }^{-} \text {radical generation }(\mathrm{PCL} \text { assay): } 1.06 \\
\text { TBARS: } 74.9 \% \text { inhibition at } 164 \mu \mathrm{M}\end{array}$ & [177] \\
\hline 155 & $\begin{array}{c}\text { C. crinita } \\
\text { (Ochrophyta, Phaeophyceae, Fucales) }\end{array}$ & $\begin{array}{l}\text { ABTS }^{+} \text {scavenging: TEAC }=0.28 \mathrm{mM} \\
\text { DPPH scavenging: } 95.8 \% \text { at } 230 \mu \mathrm{M} \\
\mathrm{O}_{2}{ }^{-} \text {radical generation (PCL assay): } 0.79 \\
\text { TBARS: } 74.6 \% \text { inhibition at } 164 \mu \mathrm{M}\end{array}$ & [177] \\
\hline 156 & $\begin{array}{c}\text { C. usneoides } \\
\text { (Ochrophyta, Phaeophyceae, Fucales) }\end{array}$ & $\mathrm{ABTS}^{+}$scavenging: $0.77 \mathrm{TE}$ & [186] \\
\hline 157 & $\begin{array}{c}\text { C. usneoides } \\
\text { (Ochrophyta, Phaeophyceae, Fucales) }\end{array}$ & $\mathrm{ABTS}^{+}$scavenging: $\mathrm{IC}_{50}=24.5 \pm 1.6 \mu \mathrm{M} ; 1.06 \mathrm{TE}$ & [178] \\
\hline 158 & $\begin{array}{c}\text { C. usneoides } \\
\text { (Ochrophyta, Phaeophyceae, Fucales) }\end{array}$ & $\mathrm{ABTS}^{+}$scavenging: $0.77 \mathrm{TE}$ & [186] \\
\hline 159 & $\begin{array}{c}\text { C. usneoides } \\
\text { (Ochrophyta, Phaeophyceae, Fucales) }\end{array}$ & ABTS $^{+}$scavenging: $\mathrm{IC}_{50}=26.3 \pm 2.3 \mu \mathrm{M} ; 0.98 \mathrm{TE}$ & [178] \\
\hline 160 & $\begin{array}{c}\text { C. usneoides } \\
\text { (Ochrophyta, Phaeophyceae, Fucales) }\end{array}$ & $\mathrm{ABTS}^{+}$scavenging: $0.87 \mathrm{TE}$ & [186] \\
\hline
\end{tabular}


Table 4. Cont.

\begin{tabular}{|c|c|c|c|}
\hline Compound & Isolation Source & Assay/Activity & Reference \\
\hline 161 & $\begin{array}{c}\text { C. usneoides } \\
\text { (Ochrophyta, Phaeophyceae, Fucales) }\end{array}$ & $\begin{array}{c}\text { ABTS }^{+} \text {scavenging: } \mathrm{IC}_{50}=33.1 \pm 5.1 \mu \mathrm{M} \\
0.78 \mathrm{TE}\end{array}$ & [178] \\
\hline 162 & $\begin{array}{c}\text { C. usneoides } \\
\text { (Ochrophyta, Phaeophyceae, Fucales) }\end{array}$ & $\mathrm{ABTS}^{+}$scavenging: $0.67 \mathrm{TE}$ & [186] \\
\hline 163 & $\begin{array}{c}\text { C. usneoides } \\
\text { (Ochrophyta, Phaeophyceae, Fucales) }\end{array}$ & $\mathrm{ABTS}^{+}$scavenging: $0.81 \mathrm{TE}$ & [186] \\
\hline 164 & $\begin{array}{c}\text { C. usneoides } \\
\text { (Ochrophyta, Phaeophyceae, Fucales) }\end{array}$ & $\begin{array}{c}\text { ABTS }^{+} \text {scavenging: } \mathrm{IC}_{50}=43.1 \pm 3.1 \mu \mathrm{M} \\
0.60 \mathrm{TE}\end{array}$ & [178] \\
\hline 165 & $\begin{array}{c}\text { C. usneoides } \\
\text { (Ochrophyta, Phaeophyceae, Fucales) }\end{array}$ & $\mathrm{ABTS}^{+}$scavenging: $0.53 \mathrm{TE}$ & [186] \\
\hline 166 & $\begin{array}{c}\text { C. usneoides } \\
\text { (Ochrophyta, Phaeophyceae, Fucales) }\end{array}$ & $\mathrm{ABTS}^{+}$scavenging: $0.37 \mathrm{TE}$ & [186] \\
\hline 167 & $\begin{array}{c}\text { C. usneoides } \\
\text { (Ochrophyta, Phaeophyceae, Fucales) }\end{array}$ & $\mathrm{ABTS}^{+}$scavenging: $0.66 \mathrm{TE}$ & [186] \\
\hline 168 & $\begin{array}{c}\text { C. usneoides } \\
\text { (Ochrophyta, Phaeophyceae, Fucales) }\end{array}$ & $\mathrm{ABTS}^{+}$scavenging: $0.45 \mathrm{TE}$ & [186] \\
\hline 169 & $\begin{array}{c}\text { C. usneoides } \\
\text { (Ochrophyta, Phaeophyceae, Fucales) }\end{array}$ & $\mathrm{ABTS}^{+}$scavenging: $0.65 \mathrm{TE}$ & {$[186]$} \\
\hline 170 & $\begin{array}{c}\text { C. usneoides } \\
\text { (Ochrophyta, Phaeophyceae, Fucales) }\end{array}$ & $\mathrm{ABTS}^{+}$scavenging: $0.50 \mathrm{TE}$ & [186] \\
\hline 171 & $\begin{array}{c}\text { C. usneoides } \\
\text { (Ochrophyta, Phaeophyceae, Fucales) }\end{array}$ & $\mathrm{ABTS}^{+}$scavenging: $0.62 \mathrm{TE}$ & {$[186]$} \\
\hline 172 & $\begin{array}{c}\text { C. usneoides } \\
\text { (Ochrophyta, Phaeophyceae, Fucales) }\end{array}$ & $\begin{array}{c}\text { ABTS }^{+} \text {scavenging: } \mathrm{IC}_{50}=24.4 \pm 0.9 \mu \mathrm{M} \\
1.06 \mathrm{TE}\end{array}$ & [178] \\
\hline 173 & $\begin{array}{c}\text { C. usneoides } \\
\text { (Ochrophyta, Phaeophyceae, Fucales) }\end{array}$ & $\begin{array}{c}\text { ABTS }^{+} \text {scavenging: } \mathrm{IC}_{50}=22.5 \pm 2.1 \mu \mathrm{M} ; \\
1.15 \mathrm{TE}\end{array}$ & [178] \\
\hline 174 & $\begin{array}{c}\text { Sargassum siliquastrum } \\
\text { (Ochrophyta, Phaeophyceae, Fucales) }\end{array}$ & DPPH scavenging: $\mathrm{IC}_{50}=0.54 \mu \mathrm{M}$ & [187] \\
\hline 175 & $\begin{array}{c}\text { Sargassum elegans, S. siliquastrum, Sargassum } \\
\text { thunbergii } \\
\text { (Ochrophyta, Phaeophyceae, Fucales) }\end{array}$ & $\begin{array}{c}\text { DPPH scavenging: } \mathrm{IC}_{50}=0.40 ; 46.9 \mu \mathrm{M} \\
\text { ONOO scavenging: } 78.03 \% \text { at } 23.4 \mu \mathrm{M} \\
\text { ONOO }^{-} \text {derived from SIN-1 scavenging: } \\
100 \% \text { at } 23.4 \mu \mathrm{M} \\
\text { electrochemistry-guided isolation of } \\
\text { antioxidant metabolites (using square wave } \\
\text { and cyclic voltammetry methods) }\end{array}$ & {$[157,187-189]$} \\
\hline 176 & $\begin{array}{c}\text { S. micracanthum } \\
\text { (Ochrophyta, Phaeophyceae, Fucales) }\end{array}$ & $\begin{array}{l}\text { DPPH scavenging: } 52.6 \% \text { inhibition at } \\
143.6 \mu \mathrm{M} \\
\text { lipid peroxidation in rat liver: } \mathrm{IC}_{50}=63.6 \mu \mathrm{M}\end{array}$ & [184] \\
\hline 177 & $\begin{array}{c}\text { S. micracanthum } \\
\text { (Ochrophyta, Phaeophyceae, Fucales) }\end{array}$ & $\begin{array}{l}\text { DPPH scavenging: } 32.3 \% \text { inhibition at } \\
144.0 \mu \mathrm{M} \\
\text { lipid peroxidation in rat liver: } \mathrm{IC}_{50}=1.66 \mu \mathrm{M}\end{array}$ & [184] \\
\hline 178 & $\begin{array}{c}\text { S. siliquastrum } \\
\text { (Ochrophyta, Phaeophyceae, Fucales) }\end{array}$ & DPPH scavenging: $\mathrm{IC}_{50}=0.27 \mu \mathrm{M}$ & [187] \\
\hline 179 & $\begin{array}{c}\text { S. siliquastrum } \\
\text { (Ochrophyta, Phaeophyceae, Fucales) }\end{array}$ & DPPH scavenging: $\mathrm{IC}_{50}=0.25 \mu \mathrm{M}$ & [187] \\
\hline 180 & $\begin{array}{c}\text { S. siliquastrum } \\
\text { (Ochrophyta, Phaeophyceae, Fucales) }\end{array}$ & DPPH scavenging: $\mathrm{IC}_{50}=0.68 \mu \mathrm{M}$ & [187] \\
\hline
\end{tabular}


Table 4. Cont.

\begin{tabular}{|c|c|c|c|}
\hline Compound & Isolation Source & Assay/Activity & Reference \\
\hline 181 & $\begin{array}{c}\text { S. siliquastrum } \\
\text { (Ochrophyta, Phaeophyceae, Fucales) }\end{array}$ & DPPH scavenging: $\mathrm{IC}_{50}=0.64 \mu \mathrm{M}$ & [187] \\
\hline 182 & $\begin{array}{c}\text { S. siliquastrum } \\
\text { (Ochrophyta, Phaeophyceae, Fucales) }\end{array}$ & DPPH scavenging: $\mathrm{IC}_{50}=0.62 \mu \mathrm{M}$ & [187] \\
\hline 183 & $\begin{array}{c}\text { S. siliquastrum } \\
\text { (Ochrophyta, Phaeophyceae, Fucales) }\end{array}$ & DPPH scavenging: $\mathrm{IC}_{50}=0.21 \mu \mathrm{M}$ & [187] \\
\hline 184 & $\begin{array}{c}\text { S. siliquastrum } \\
\text { (Ochrophyta, Phaeophyceae, Fucales) }\end{array}$ & DPPH scavenging: $\mathrm{IC}_{50}=23.3 \mu \mathrm{M}$ & [187] \\
\hline 185 & $\begin{array}{c}\text { S. siliquastrum } \\
\text { (Ochrophyta, Phaeophyceae, Fucales) }\end{array}$ & DPPH scavenging: $\mathrm{IC}_{50}=26.1 \mu \mathrm{M}$ & [187] \\
\hline 186 & $\begin{array}{c}\text { S. siliquastrum } \\
\text { (Ochrophyta, Phaeophyceae, Fucales) }\end{array}$ & DPPH scavenging: $\mathrm{IC}_{50}=25.4 \mu \mathrm{M}$ & [187] \\
\hline 187 & $\begin{array}{c}\text { S. siliquastrum } \\
\text { (Ochrophyta, Phaeophyceae, Fucales) }\end{array}$ & DPPH scavenging: $\mathrm{IC}_{50}=37.9 \mu \mathrm{M}$ & [187] \\
\hline 188 & $\begin{array}{c}\text { S. siliquastrum } \\
\text { (Ochrophyta, Phaeophyceae, Fucales) }\end{array}$ & DPPH scavenging: $\mathrm{IC}_{50}=35.4 \mu \mathrm{M}$ & [187] \\
\hline 189 & $\begin{array}{c}\text { S. siliquastrum } \\
\text { (Ochrophyta, Phaeophyceae, Fucales) }\end{array}$ & DPPH scavenging: $\mathrm{IC}_{50}=18.7 \mu \mathrm{M}$ & [187] \\
\hline 190 & $\begin{array}{c}\text { S. siliquastrum } \\
\text { (Ochrophyta, Phaeophyceae, Fucales) }\end{array}$ & DPPH scavenging: $\mathrm{IC}_{50}=25.9 \mu \mathrm{M}$ & [187] \\
\hline 191 & $\begin{array}{c}\text { S. siliquastrum } \\
\text { (Ochrophyta, Phaeophyceae, Fucales) }\end{array}$ & DPPH scavenging: $\mathrm{IC}_{50}=30.4 \mu \mathrm{M}$ & [187] \\
\hline 192 & $\begin{array}{c}\text { S. siliquastrum } \\
\text { (Ochrophyta, Phaeophyceae, Fucales) }\end{array}$ & DPPH scavenging: $\mathrm{IC}_{50}=47.9 \mu \mathrm{M}$ & [187] \\
\hline 193 & $\begin{array}{c}\text { S. siliquastrum } \\
\text { (Ochrophyta, Phaeophyceae, Fucales) }\end{array}$ & DPPH scavenging: $\mathrm{IC}_{50}=26.3 \mu \mathrm{M}$ & [187] \\
\hline 194 & $\begin{array}{c}\text { S. siliquastrum } \\
\text { (Ochrophyta, Phaeophyceae, Fucales) }\end{array}$ & DPPH scavenging: $\mathrm{IC}_{50}=25.1 \mu \mathrm{M}$ & [187] \\
\hline 195 & $\begin{array}{c}\text { S. micracanthum } \\
\text { (Ochrophyta, Phaeophyceae, Fucales) }\end{array}$ & $\begin{array}{c}\text { DPPH scavenging: } \mathrm{IC}_{50}=933.3 \mu \mathrm{M} \\
\text { lipid peroxidation in rat liver: } \mathrm{IC}_{50}=2.33 \mu \mathrm{M}\end{array}$ & [183] \\
\hline 196 & $\begin{array}{c}\text { S. elegans } \\
\text { (Ochrophyta, Phaeophyceae, Fucales) }\end{array}$ & $\begin{array}{l}\text { electrochemistry-guided isolation of } \\
\text { antioxidant metabolites (using square wave } \\
\text { and cyclic voltammetry methods) }\end{array}$ & [157] \\
\hline 197 & $\begin{array}{l}\text { S. elegans, S. micracanthum, S. thunbergii } \\
\text { (Ochrophyta, Phaeophyceae, Fucales) }\end{array}$ & 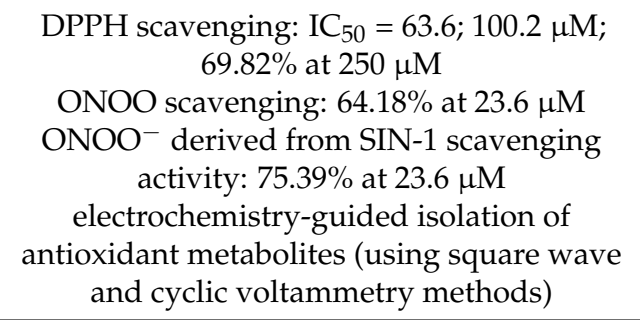 & {$[157,188-190]$} \\
\hline 198 & $\begin{array}{c}\text { C. crinita } \\
\text { (Ochrophyta, Phaeophyceae, Fucales) }\end{array}$ & $\begin{array}{c}\text { ABTS }^{+} \text {scavenging } \\
\text { DPPH scavenging: } 29.0 \% \text { at } 230 \mu \mathrm{M} \\
\mathrm{O}_{2}^{-} \text {generation (PCL assay) } \\
\text { TBARS: } 43.3 \% \text { inhibition at } 164 \mu \mathrm{M}\end{array}$ & [177] \\
\hline 199 & $\begin{array}{c}\text { C. crinita } \\
\text { (Ochrophyta, Phaeophyceae, Fucales) }\end{array}$ & $\begin{array}{c}\text { ABTS }^{+} \text {scavenging: TEAC }=0.30 \mathrm{mM} \\
\text { DPPH scavenging: } 38.6 \% \text { at } 230 \mu \mathrm{M} \\
\mathrm{O}_{2}^{-} \text {generation (PCL assay): } 1.41 \\
\text { TBARS: } 54.4 \% \text { inhibition at } 164 \mu \mathrm{M}\end{array}$ & [177] \\
\hline
\end{tabular}


Table 4. Cont.

\begin{tabular}{|c|c|c|c|}
\hline Compound & Isolation Source & Assay/Activity & Reference \\
\hline 200 & $\begin{array}{c}\text { C. barbata } \\
\text { (Ochrophyta, Phaeophyceae, Fucales) }\end{array}$ & $\begin{array}{c}\text { antioxidant activity against ROS and reactive } \\
\text { nitrogen species }\end{array}$ & $\begin{array}{c}{[141,183,184,} \\
189]\end{array}$ \\
\hline 201 & $\begin{array}{c}\text { S. siliquastrum } \\
\text { (Ochrophyta, Phaeophyceae, Fucales) }\end{array}$ & DPPH scavenging: $90.0 \%$ at $0.29 \mathrm{mM}$ & [191] \\
\hline 202 & $\begin{array}{c}\text { S. siliquastrum } \\
\text { (Ochrophyta, Phaeophyceae, Fucales) }\end{array}$ & DPPH scavenging: $87.4 \%$ at $0.29 \mathrm{mM}$ & [191] \\
\hline 203 & $\begin{array}{c}\text { S. siliquastrum } \\
\text { (Ochrophyta, Phaeophyceae, Fucales) }\end{array}$ & $\begin{array}{l}\mathrm{H}_{2} \mathrm{O}_{2} \text {-induced lipid peroxidation in HT } \\
1080 \text { cells } \\
\text { intracellular GSH level in HT } 1080 \text { cells } \\
\text { intracellular ROS generation (DCFH-DA) in } \\
\text { HT } 1080 \text { cells }\end{array}$ & [192] \\
\hline 204 & $\begin{array}{c}\text { S. siliquastrum } \\
\text { (Ochrophyta, Phaeophyceae, Fucales) }\end{array}$ & DPPH scavenging: $90.5 \%$ at $0.24 \mathrm{mM}$ & [191] \\
\hline 205 & $\begin{array}{c}\text { S. siliquastrum } \\
\text { (Ochrophyta, Phaeophyceae, Fucales) }\end{array}$ & $\begin{array}{c}\text { DPPH scavenging: } 89.6 \% \text { at } 0.23 \mathrm{mM} \\
\mathrm{H}_{2} \mathrm{O}_{2} \text {-induced lipid peroxidation in HT } \\
1080 \text { cells } \\
\text { intracellular GSH level in HT } 1080 \text { cells } \\
\text { intracellular ROS generation (DCFH-DA) in } \\
\text { HT } 1080 \text { cells: } 67.2 \% \text { decrease at } 11.7 \mu \mathrm{M}\end{array}$ & {$[191,192]$} \\
\hline 206 & $\begin{array}{c}\text { S. siliquastrum } \\
\text { (Ochrophyta, Phaeophyceae, Fucales) }\end{array}$ & $\begin{array}{l}\text { DPPH scavenging: } 87.3 \% \text { at } 0.23 \mathrm{mM} \\
\mathrm{H}_{2} \mathrm{O}_{2} \text {-induced lipid peroxidation in HT } \\
1080 \text { cells } \\
\text { intracellular GSH level in HT } 1080 \text { cells } \\
\text { intracellular ROS generation (DCFH-DA) in } \\
\text { HT } 1080 \text { cells: } 87.2 \% \text { decrease at } 11.7 \mu \mathrm{M}\end{array}$ & {$[191,192]$} \\
\hline 207 & $\begin{array}{c}\text { S. siliquastrum } \\
\text { (Ochrophyta, Phaeophyceae, Fucales) }\end{array}$ & DPPH scavenging: $88.2 \%$ at $0.23 \mathrm{mM}$ & [191] \\
\hline 208 & $\begin{array}{c}\text { S. siliquastrum } \\
\text { (Ochrophyta, Phaeophyceae, Fucales) }\end{array}$ & $\begin{array}{c}\text { DPPH scavenging: } 90.4 \% \text { at } 0.23 \mathrm{mM} \\
\text { expression of osteoclastic marker gene in } \\
\text { RANKL-stimulated RAW264.7 cells (TRAP, } \\
\text { CTSK, MMP9 and CTR) } \\
\text { NF-KB activation in RANKL-stimulated } \\
\text { RAW264.7 cells } \\
\text { osteoclast differentiation in } \\
\text { RANKL-stimulated RAW264.7 cells } \\
\text { phosphorylation of MAPKs in } \\
\text { RANKL-stimulated RAW264.7 cells }\end{array}$ & {$[191,193]$} \\
\hline 209 & $\begin{array}{c}\text { S. siliquastrum } \\
\text { (Ochrophyta, Phaeophyceae, Fucales) }\end{array}$ & $\begin{array}{c}\text { DPPH scavenging: } 89.2 \% \text { at } 0.23 \mathrm{mM} \\
\mathrm{H}_{2} \mathrm{O}_{2} \text {-induced lipid peroxidation in HT } \\
1080 \text { cells } \\
\text { intracellular GSH level in HT } 1080 \text { cells } \\
\text { intracellular ROS generation (DCFH-DA } \\
\text { assay) in HT } 1080 \text { cells }\end{array}$ & {$[191,192]$} \\
\hline 210 & $\begin{array}{c}\text { S. siliquastrum } \\
\text { (Ochrophyta, Phaeophyceae, Fucales) }\end{array}$ & DPPH scavenging: $87.8 \%$ at $0.23 \mathrm{mM}$ & [191] \\
\hline 211 & $\begin{array}{c}\text { S. siliquastrum } \\
\text { (Ochrophyta, Phaeophyceae, Fucales) }\end{array}$ & DPPH scavenging: $90.4 \%$ at $0.23 \mathrm{mM}$ & [191] \\
\hline 212 & $\begin{array}{c}\text { S. siliquastrum } \\
\text { (Ochrophyta, Phaeophyceae, Fucales) }\end{array}$ & DPPH scavenging: $89.1 \%$ at $0.23 \mathrm{mM}$ & [191] \\
\hline
\end{tabular}


Table 4. Cont.

\begin{tabular}{|c|c|c|c|}
\hline Compound & Isolation Source & Assay/Activity & Reference \\
\hline 213 & $\begin{array}{c}\text { S. micracanthum } \\
\text { (Ochrophyta, Phaeophyceae, Fucales) }\end{array}$ & $\begin{array}{l}\text { NADPH-dependent lipid peroxidation in rat } \\
\text { microsomes: } \mathrm{IC}_{50}=0.65 \mu \mathrm{M}\end{array}$ & [194] \\
\hline 214 & $\begin{array}{c}\text { S. micracanthum, S. thunbergii } \\
\text { (Ochrophyta, Phaeophyceae, Fucales) }\end{array}$ & $\begin{array}{c}\text { DPPH scavenging: } \mathrm{IC}_{50}=75.4 \mu \mathrm{M} ; 78.85 \% \text { at } \\
250 \mu \mathrm{M} \\
\text { ONOO scavenging: } 92.69 \% \text { at } 23.6 \mu \mathrm{M} \\
\text { ONOO }^{-} \text {derived from SIN-1 scavenging: } 99.51 \% \\
\text { at } 23.6 \mu \mathrm{M}\end{array}$ & [188-190] \\
\hline 215 & $\begin{array}{c}\text { S. thunbergii } \\
\text { (Ochrophyta, Phaeophyceae, Fucales) }\end{array}$ & DPPH scavenging: $\mathrm{IC}_{50}=82.9 \mu \mathrm{M}$ & [189] \\
\hline 216 & $\begin{array}{c}\text { S. siliquastrum } \\
\text { (Ochrophyta, Phaeophyceae, Fucales) }\end{array}$ & $\begin{array}{c}\mathrm{H}_{2} \mathrm{O}_{2} \text {-induced lipid peroxidation in HT } \\
1080 \text { cells: } 43.2 \% \text { at } 112.0 \mu \mathrm{M} \\
\text { intracellular GSH level in HT } 1080 \text { cells } \\
\text { intracellular ROS generation (DCFH-DA) in HT } \\
1080 \text { cells }\end{array}$ & [192] \\
\hline 217 & $\begin{array}{c}\text { S. siliquastrum } \\
\text { (Ochrophyta, Phaeophyceae, Fucales) }\end{array}$ & $\begin{array}{c}\mathrm{H}_{2} \mathrm{O}_{2} \text {-induced lipid peroxidation in HT } \\
1080 \text { cells: } 38.9 \% \text { at } 112.0 \mu \mathrm{M} \\
\text { intracellular GSH level in HT } 1080 \text { cells } \\
\text { intracellular ROS generation (DCFH-DA) in HT } \\
1080 \text { cells }\end{array}$ & [192] \\
\hline 218 & $\begin{array}{c}\text { S. siliquastrum } \\
\text { (Ochrophyta, Phaeophyceae, Fucales) }\end{array}$ & DPPH scavenging: $88.8 \%$ at $0.24 \mathrm{mM}$ & [191] \\
\hline 219 & $\begin{array}{c}\text { S. thunbergii } \\
\text { (Ochrophyta, Phaeophyceae, Fucales) }\end{array}$ & $\begin{array}{c}\text { DPPH scavenging: } \mathrm{IC}_{50}=67.8 \mu \mathrm{M} \\
\text { ONOO scavenging: } 60.0 \% \text { at } 11.3 \mu \mathrm{M} \\
\text { ONOO }^{-} \text {derived from SIN-1 scavenging: } 98.6 \% \\
\text { at } 11.3 \mu \mathrm{M}\end{array}$ & [195] \\
\hline 220 & $\begin{array}{c}\text { S. thunbergii } \\
\text { (Ochrophyta, Phaeophyceae, Fucales) }\end{array}$ & $\begin{array}{c}\text { DPPH scavenging: } \mathrm{IC}_{50}=70.0 \mu \mathrm{M} \\
\text { ONOO scavenging: } 57.1 \% \text { at } 11.3 \mu \mathrm{M} \\
\text { ONOO }^{-} \text {derived from SIN-1 scavenging: } 90.6 \% \\
\text { at } 11.3 \mu \mathrm{M}\end{array}$ & [195] \\
\hline 221 & $\begin{array}{c}\text { S. siliquastrum } \\
\text { (Ochrophyta, Phaeophyceae, Fucales) }\end{array}$ & DPPH scavenging: $90.1 \%$ at $0.24 \mathrm{mM}$ & [191] \\
\hline 222 & $\begin{array}{c}\text { S. siliquastrum } \\
\text { (Ochrophyta, Phaeophyceae, Fucales) }\end{array}$ & DPPH scavenging: $88.7 \%$ at $0.23 \mathrm{mM}$ & [191] \\
\hline 223 & $\begin{array}{c}\text { S. siliquastrum } \\
\text { (Ochrophyta, Phaeophyceae, Fucales) }\end{array}$ & DPPH scavenging: $89.2 \%$ at $0.24 \mathrm{mM}$ & [191] \\
\hline 224 & $\begin{array}{c}\text { S. siliquastrum } \\
\text { (Ochrophyta, Phaeophyceae, Fucales) }\end{array}$ & DPPH scavenging: $88.7 \%$ at $0.24 \mathrm{mM}$ & [191] \\
\hline
\end{tabular}

$\mathrm{ABTS}^{+}:$2,2'-azino-bis (3-ethyl benzothiazoline-6-sulfonic acid) diammonium salt; ARE: antioxidant response element; CTR: calcitonin receptor; CTSK: cathepsin K; DCFH-DA: cell-based 2',7'-dichlorodihydrofluorescein diacetate antioxidant assay; DPPH: 1,1-diphenyl2-picrylhydrazyl free radical; GSH: glutathione; HO-1: heme oxygenase-1; HT 1080: human fibrosarcoma cell line; $\mathrm{IC}_{50}$ : half maximal inhibitory concentration; MMP9: matrix metalloproteinase 9; NADPH: nicotinamide adenine dinucleotide phosphate; NQO1: NADPH quinone oxidoreductase 1; Nrf2: nuclear factor erythroid 2-related factor 2; $\mathrm{ONOO}^{-}$: peroxynitrite; $\mathrm{O}_{2}^{-}$: superoxide anion; $\mathrm{PCL}$ : photochemiluminescence; PRDX4: peroxyredoxin 4; RANKL: receptor activator of NF-кB ligand; ROS: reactive oxygen species; SIN-1: 3-morpholinosydnonimine; SOD: superoxide dismutase; TBARS: thiobarbituric acid reactive substances; TE: trolox equivalents; TEAC: trolox equivalence antioxidant capacity; TPA: 12-O-tetradecanoylphorbol 13-acetate; TRAP: tartrate-resistant acid phosphatase.

Overall, meroterpenoids from marine macroalgae have exhibited moderate to remarkable antioxidant activity. Specifically, the brominated compound cymopol (125), isolated from the green alga Cymopolia barbata, exerted noticeably high DPPH scavenging activity with an $\mathrm{IC}_{50}$ value of $4.0 \mu \mathrm{M}$ [91].

De los Reyes et al. $(2013,2016)$ described the isolation of meroditerpenoids 129-132 and 156-173 that have shown radical scavenging activity from the brown alga Cystoseira usneoides [178,186]. The most active compounds were cystodiones A (173), B (172), G (162), and H (158), cystomexicone B (129), amentadione (156), amentadione $1^{\prime}$-methyl ether 
(157), 6-cis-amentadione 1'-methyl ether (159), and 11-hydroxyamentadione (160), which exhibited antioxidant activity in the ABTS assay in the range of $77-115 \%$ compared to Trolox that was used as a standard.<smiles>CC(C)=CCC/C(C)=C/Cc1cc(O)c(Br)cc1O</smiles><smiles>CCCCCC(=O)CC(C)=CCc1cc(O)cc(C)c1O</smiles>

127<smiles>COc1c(C)cc(O)cc1CC=C(C)CC(=O)C=C(C)CCCC(C)=O</smiles>

129<smiles>COc1c(C)cc(O)cc1/C=C/C(C)(O)CC(=O)/C=C(\C)CCCC(C)=O</smiles>

131<smiles>CC1=CC[C@@H]2[C@@H](CCCC2(C)C)C1Cc1cc(O)ccc1O</smiles>

133<smiles></smiles>

134<smiles>CC(=CCc1cc(O)c(Br)cc1O)CCCC(C)(C)O</smiles><smiles>CC(C)=CCC/C(C)=C\C(=O)C/C(C)=C/Cc1cc(O)cc(C)c1O</smiles>

128<smiles>COc1c(C)cc(O)cc1C/C=C(\C)CC(=O)/C=C(/C)CCCC(C)=O</smiles><smiles>COc1c(C)cc(O)cc1/C=C/C(C)(O)CC(=O)/C=C(/C)CCCC(C)=O</smiles>

132<smiles>COC1CC[C@H]2C(C)(C)CC[C@@H]3Oc4ccc(O)cc4[C@@]3(C)[C@@]12C</smiles>

137<smiles>C=CCCCO[C@H]1CC[C@]2(C)C3CC[C@@](C)(Oc4ccc(O)cc4[C@H]2O)O[C@]31C</smiles>

138<smiles>COC(c1cccc(O)c1)C1C(C)(OC)CC[C@H]2C(C)(C)CCC[C@]12C</smiles>

136<smiles>CC1=CC[C@@H]2[C@@H](CCCC2(C)C)C1CC1=CC(=O)C=CC1=O</smiles>

139

Figure 13. Chemical structures of compounds 125-139. 
<smiles>C=C1[C@@H](OC(=O)CCCC)CCC[C@@]1(C)CC[C@]1(C)C=Cc2c(OC)c(C)c(C)c(O)c2O1</smiles><smiles>[R]C(CC(O)C(C)(C)O)C(C)=CCCC(C)=CC(=O)CC(C)=CCc1cc(OC)cc(C)c1OC</smiles>

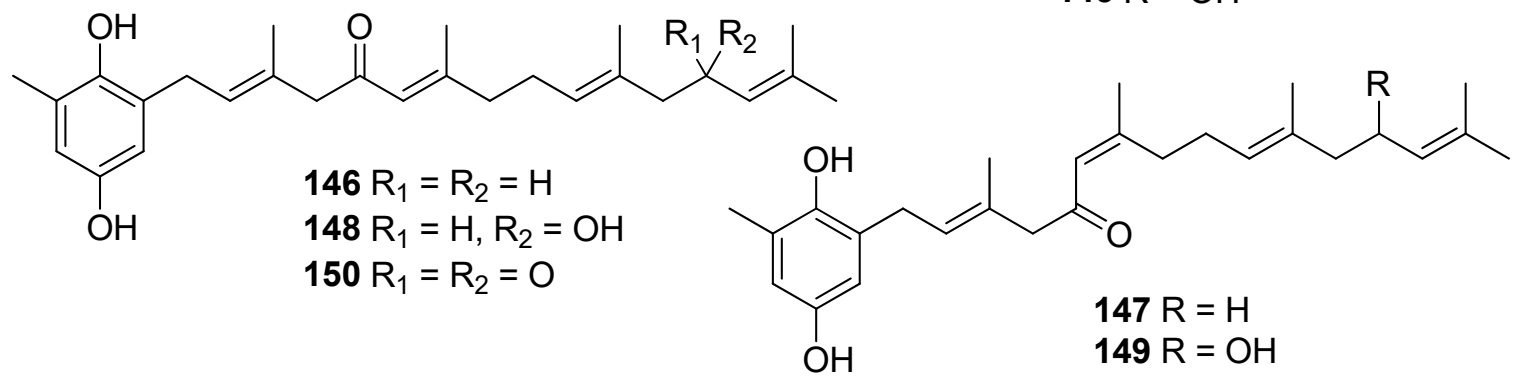<smiles>[R][R](=O)C=[R16]([R16])[Y15]</smiles><smiles>C/C(=C\C/C=C(\C)CC/C(C)=C/C(=O)C/C(C)=C/Cc1cc(O)cc(C)c1O)C/C=C(\C)CC/C=C(\C)CC(=O)/C=C(\C)Cc1cc(C)co1</smiles><smiles>[R2]c1c(C)cc(O)cc1C/C=C(\C)CC(=O)/C=C(\C)CCCC(C)C(=O)/C=C/C(C)(C)O</smiles>

Figure 14. Chemical structures of compounds 140-157. 
<smiles>[R2]c1c(CC=C(C)CC(=O)C=C(C)CCCC(C)C(=O)C=CC(C)(C)O)cc(O)cc1CC=C(C)CC(=O)C=C(C)CCCC(C)(O)C(=O)C=CC(C)(C)O</smiles><smiles>COc1c(C)cc(O)cc1C/C=C(\C)CC(=O)/C=C(\C)CCC[C@]1(C)O[C@@H](C(C)(C)O)CC1=O</smiles>

Figure 15. Chemical structures of compounds 158-171. 
<smiles>COc1c(C)cc(O)cc1/C=C/C(C)(O)CC(=O)/C=C(\C)CCCC(C)C(=O)/C=C/C(C)(C)O</smiles>

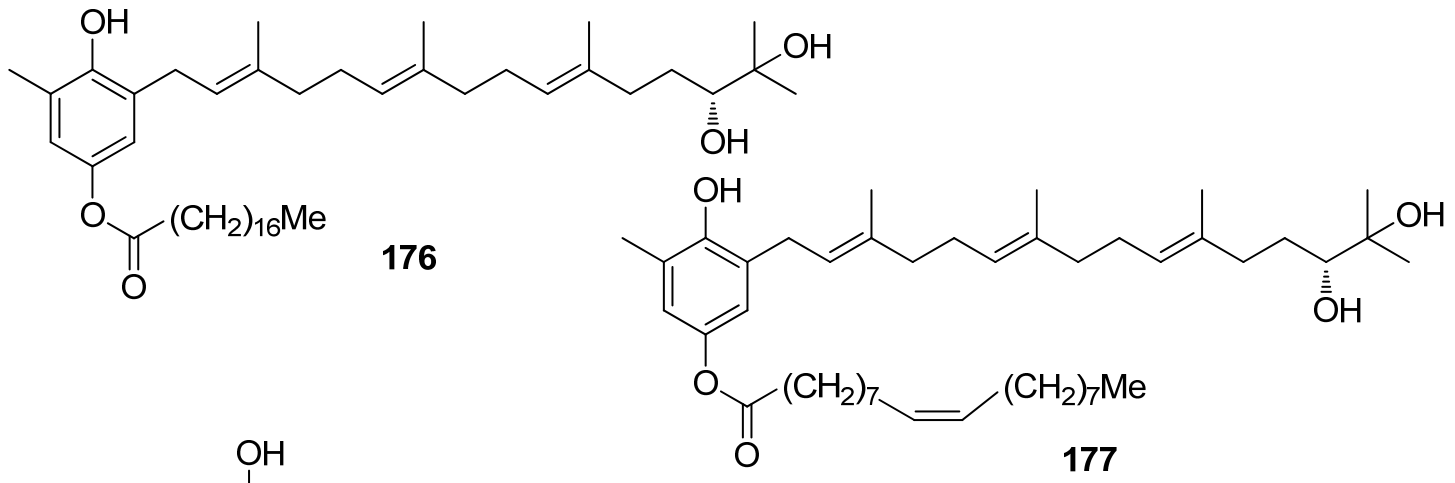<smiles>[R]C([R2])(C=C(C)C)C([R2])([R])C(C)=CCCC(C)=CCCC(C)=CCc1cc(O)cc(CC(=O)OC)c1O</smiles>

$178 \mathrm{R}_{1}=\mathrm{OH}, \mathrm{R}_{2}=\mathrm{R}_{3}=\mathrm{H}, \mathrm{R}_{4}=\mathrm{OH}$

$180 \mathrm{R}_{1}=\mathrm{R}_{2}=\mathrm{O}, \mathrm{R}_{3}=\mathrm{OH}, \mathrm{R}_{4}=\mathrm{H} \mathrm{OH}$<smiles>[R]C([R2])(C=C(C)C)C([R2])([R2])/C(C)=C/CC/C(C)=C/CC/C(C)=C/Cc1cc(O)cc(CC(=O)OC)c1O</smiles>

$179 \mathrm{R}_{1}=\mathrm{OH}, \mathrm{R}_{2}=\mathrm{R}_{3}=\mathrm{H}, \mathrm{R}_{4}=\mathrm{OH}$

$181 \mathrm{R}_{1}=\mathrm{R}_{2}=\mathrm{O}, \mathrm{R}_{3}=\mathrm{OH}, \mathrm{R}_{4}=\mathrm{H}$

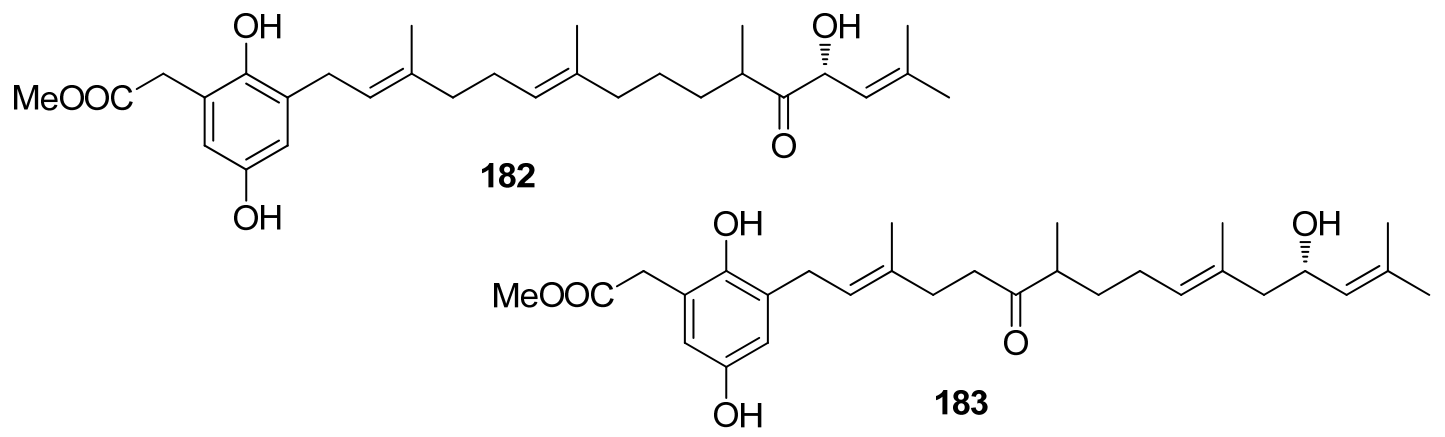

Figure 16. Chemical structures of compounds 172-183. 


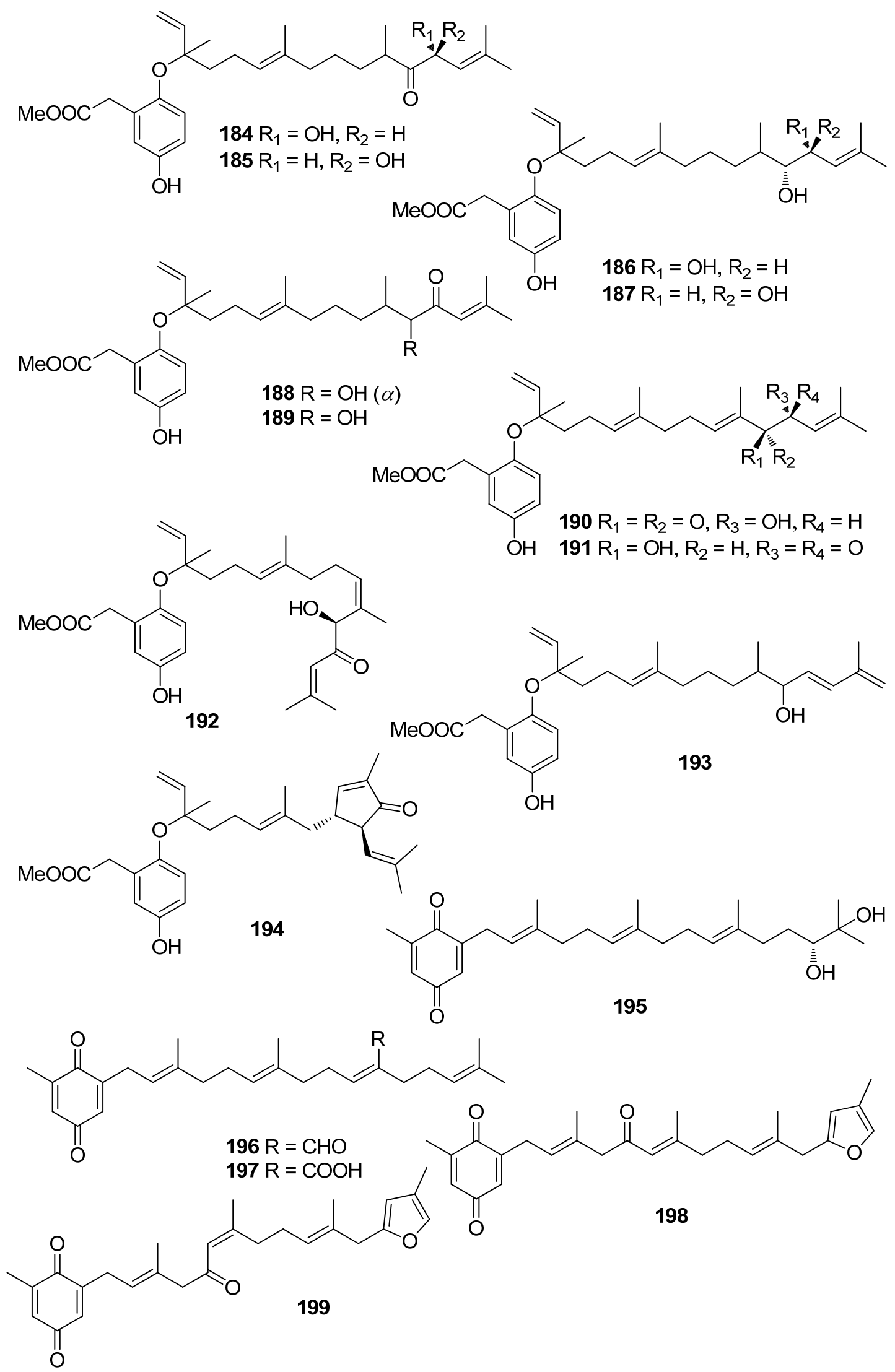

Figure 17. Chemical structures of compounds 184-199. 


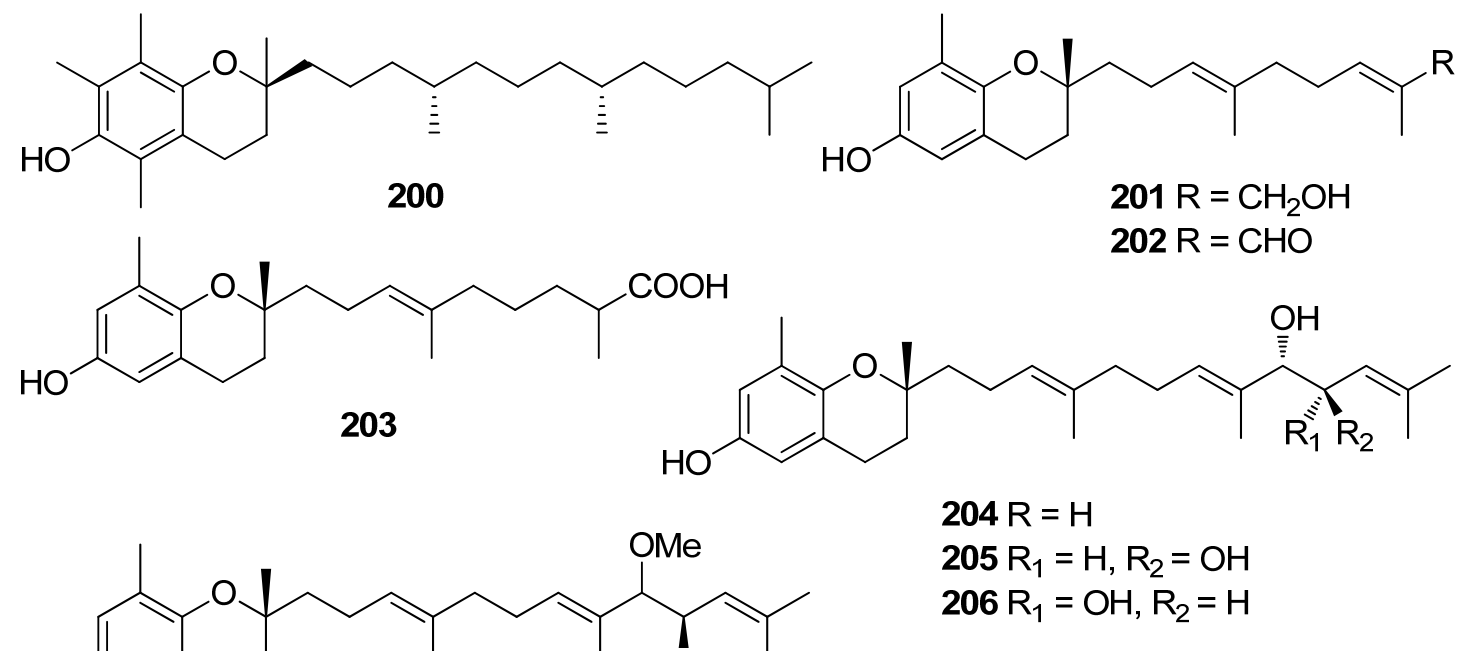<smiles>[R]C([R3])(C=C(C)C)C([R])([R])/C(C)=C/CC/C(C)=C/CC[C@]1(C)CCc2cc(O)cc(C)c2O1</smiles>

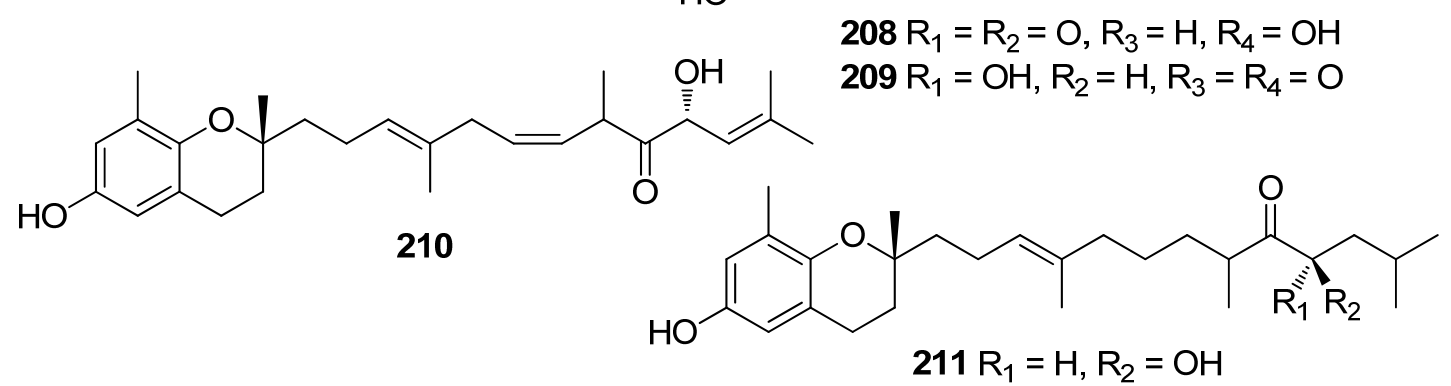<smiles>CC(=CCC[C@]1(C)C=Cc2cc(O)cc(C)c2O1)CCC=C(C)CC[C@@H](O)C(C)(C)O</smiles>

$212 \mathrm{R}_{1}=\mathrm{OH}, \mathrm{R}_{2}=\mathrm{H}$<smiles>[Z14]C(=CCCC1(C)C=Cc2cc(O)cc(C)c2O1)CCC=C(CCC=C(C)C)C(=O)O</smiles><smiles>[Z15]C(=CCCC1(C)C=Cc2cc(O)cc(C)c2O1)CCC=C(CCC(O)C(C)(C)O)C(=O)O</smiles>

Figure 18. Chemical structures of compounds 200-215.

Additionally, Fisch et al. (2003) reported a number of triprenyltoluquinol derivatives $(127,128,146-155)$, isolated from the brown alga Cystoseira crinita, that showed very high levels of radical scavenging at a concentration of $230 \mu \mathrm{M}(92.5-96.7 \%$ as compared to $95.2 \%$ scavenging for $\alpha$-tocopherol) [177]. In contrast, the co-occurring quinones 197 and 198 showed DPPH radical scavenging activities significantly less than that of $\alpha$-tocopherol and the hydroquinones, but still comparable to that of BHT, i.e., $29.0 \%$ for 197 and $38.6 \%$ for 198 as compared to $35.6 \%$ scavenging observed for BHT at a concentration of $230 \mu \mathrm{M}$. The observed differences in the values obtained in the DPPH assay for the tested compounds were attributed to the existence of small impurities in the samples (e.g., due to autoxidation) and the handling of small amounts rather than to structural variations. On the other hand, 
in the TBARS assay, potent inhibition of linolenic acid methyl ester peroxidation was observed for all hydroquinones, i.e., 66.5-74.9\% inhibition for compounds 127, 128, and 146-155 at a concentration of $164 \mu \mathrm{M}$. These activities were comparable to those of $\alpha$ tocopherol (72.7\%) and BHT (69.3\%). Additionally, these compounds showed activities between 13\% (153) and 59\% (149) of $\alpha$-tocopherol in the TEAC test and between $40 \%(\mathbf{1 5 2})$ and $112 \%$ (198) of $\alpha$-tocopherol in the PCL assay [177].

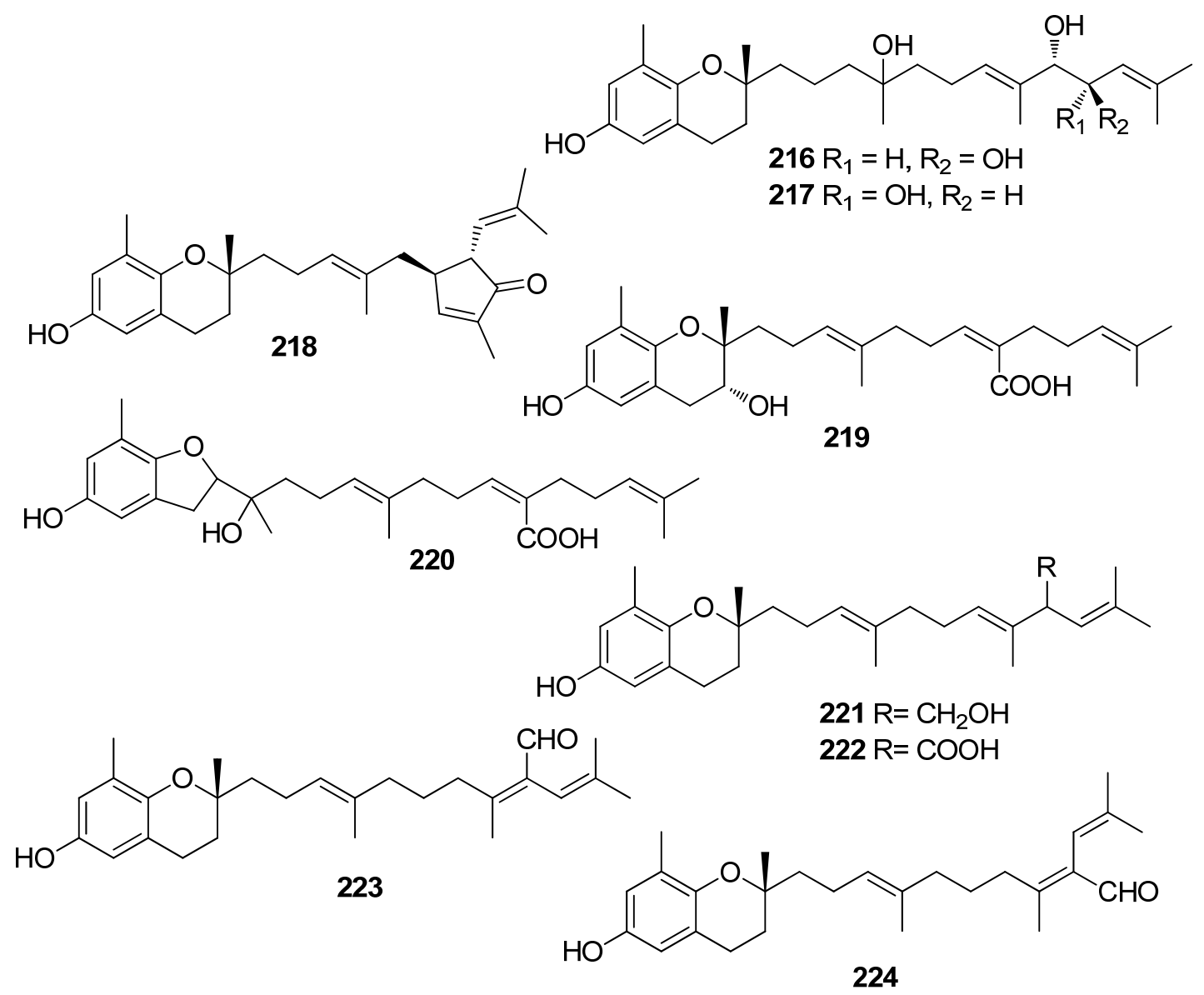

Figure 19. Chemical structures of compounds 216-224.

Jung et al. (2008) isolated an array of meroterpenoids (174, 175, 178-194) from the brown alga Sargassum siliquastrum which exhibited moderate to significant radical scavenging activity in the $\mathrm{DPPH}$ assay with $\mathrm{IC}_{50}$ values ranging from 0.21 to $47.9 \mu \mathrm{M}$ (for compounds 183 and 192, respectively) [187]. The observed more than 200-fold increase in the radical scavenging activity of the isonahocols $\left(\mathbf{1 7 4}, \mathbf{1 7 5}, \mathbf{1 7 8}-\mathbf{1 8 3}\right.$ with $\mathrm{IC}_{50}$ values of $0.54,0.40,0.27,0.25,0.64,0.68,0.62$, and $0.21 \mu \mathrm{M}$, respectively) in comparison to that of the nahocols (184-194 with $\mathrm{IC}_{50}$ values of 23.3, 26.1, 25.4, 37.9, 35.4, 18.7, 25.9, 30.4, 47.9, 26.3, and $25.1 \mu \mathrm{M}$, respectively) indicated the pivotal role of the second free hydroxyl group in the phenol ring for enhanced radical scavenging activity. Along this trend, the absence of a free phenolic hydroxyl group resulted in lack of scavenging activity [187].

Another investigation conducted by Jang et al. (2005) reported the isolation of meroterpenoids belonging to the subclasses of chromenes and chromenols $(201,202,204-212,218$, 221-224) from the brown alga $S$. siliquastrum that exhibited over $87 \%$ radical scavenging activity at a concentration of 0.23 to $0.29 \mathrm{mM}(0.1 \mathrm{mg} / \mathrm{mL})$ [191]. Moreover, the antioxidant activity of compounds 205, 206, and 209, along with that of 203, 216, and 217, was evaluated in various assays, including scavenging effects on the generation of intracellular ROS, increments of intracellular GSH levels, and inhibitory effects on lipid peroxidation in human fibrosarcoma HT 1080 cells [192]. All tested compounds significantly decreased the 
generation of intracellular ROS, while increasing the levels of intracellular GSH at a concentration of $5 \mu \mathrm{g} / \mathrm{mL}$, and inhibited $\mathrm{H}_{2} \mathrm{O}_{2}$-induced lipid peroxidation at a concentration of $50 \mu \mathrm{g} / \mathrm{mL}$.

In an effort to elucidate the mechanism of antioxidant activity of zonarol (134), Shimizu et al. (2015) studied its effect on neuronal cells and proved that zonarol protects them from oxidative stress by activating the Nrf2/ARE pathway and inducing phase-2 enzymes [180].

Moreover, Yoon et al. (2013) elucidated the role of sargachromanol G (208), isolated from the brown alga S. siliquastrum, in receptor activator of NF-KB ligand (RANKL)induced osteoclast formation [193]. Compound 208 was found to inhibit RANKL-induced osteoclast differentiation from RAW264.7 cells without signs of cytotoxicity. Additionally, the expression of osteoclastic marker genes, such as tartrate-resistant acid phosphatase (TRAP), cathepsin K (CTSK), matrix metalloproteinase 9 (MMP9), and calcitonin receptor (CTR), was also strongly inhibited. It was concluded that sargachromanol $G$ inhibits RANKL-induced activation of NF- $\kappa B$ by suppressing RANKL-mediated I $\kappa \mathrm{B}-\alpha$ protein degradation, and therefore the phosphorylation of mitogen activated protein kinases (p38, JNK, and ERK).

\section{Nitrogenous Compounds}

So far, a number of nitrogenous compounds, including peptides, alkaloids, and chlorophyll-related pigments (Figures 20 and 21), isolated from marine macroalgae have shown antioxidant activity (Table 5).

Table 5. Nitrogenous compounds from macroalgae with antioxidant activity.

\begin{tabular}{|c|c|c|c|}
\hline Compound & Isolation Source & Assay/Activity & Reference \\
\hline 225 & $\begin{array}{c}\text { Porphyra yezoensis } \\
\text { (Rhodophyta, Bangiophyceae, Bangiales) }\end{array}$ & $\begin{array}{c}\text { DPPH scavenging: } \mathrm{IC}_{50}=185.2 \pm 3.2 \mu \mathrm{M} \\
\text { ORAC: } 51 \pm 7 \% \text { TE } \\
\text { Nrf2-regulated antioxidant response in } \\
\text { UVA-treated fibroblasts (1BR) }\end{array}$ & {$[196,197]$} \\
\hline 226 & $\begin{array}{c}\text { G. furcata } \\
\text { (Rhodophyta, Florideophyceae, Gigartinales) }\end{array}$ & $\begin{array}{c}\text { DPPH scavenging: } \mathrm{IC}_{50}=399.0 \pm 1.1 \mu \mathrm{M} \\
\text { ORAC: } 17 \pm 7 \% \mathrm{TE} \\
\text { Nrf2-regulated antioxidant response in } \\
\text { UVA-treated fibroblasts (1BR) }\end{array}$ & [196] \\
\hline 227 & $\begin{array}{c}\text { P. yezoensis } \\
\text { (Rhodophyta, Bangiophyceae, Bangiales) }\end{array}$ & DPPH scavenging: $\mathrm{IC}_{50}=30.8 \mu \mathrm{M}$ & [197] \\
\hline 228 & $\begin{array}{c}\text { P. yezoensis } \\
\text { (Rhodophyta, Bangiophyceae, Bangiales) }\end{array}$ & $\begin{array}{l}\text { TBARS: } 85.2 \% \text { inhibition } \\
\text { FTC: } 84.1 \% \text { inhibition }\end{array}$ & [198] \\
\hline 229 & $\begin{array}{c}\text { P. yezoensis } \\
\text { (Rhodophyta, Bangiophyceae, Bangiales) }\end{array}$ & $\begin{array}{l}\text { TBARS: } 94.4 \% \text { inhibition } \\
\text { FTC: } 89.1 \% \text { inhibition }\end{array}$ & [198] \\
\hline 230 & $\begin{array}{c}\text { Martensia fragilis } \\
\text { (Rhodophyta, Florideophyceae, Ceramiales) }\end{array}$ & $\begin{array}{l}\text { DPPH scavenging: moderate } \\
\text { exogenous ROS scavenging in TPA-treated } \\
\text { HL-60 cells (DCFH-DA): } \mathrm{IC}_{50}=11 \mu \mathrm{M}\end{array}$ & [91] \\
\hline 231 & $\begin{array}{c}\text { Dictyota coriacea } \\
\text { (Ochrophyta, Phaeophyceae, Dictyotales) }\end{array}$ & $\begin{array}{c}\mathrm{H}_{2} \mathrm{O}_{2} \text {-induced oxidative damage and toxicity in } \\
\text { neuron-like PC12 cell } \\
\text { Nrf2/ARE signaling pathway }\end{array}$ & [199] \\
\hline 232 & $\begin{array}{c}\text { Porphyra dioica } \\
\text { (Rhodophyta, Bangiophyceae, Bangiales) }\end{array}$ & ORAC: $3.79 \pm 0.11 \mu \mathrm{mol} \mathrm{TE} / \mu \mathrm{M}$ & [200] \\
\hline 233 & $\begin{array}{c}\text { P. dioica } \\
\text { (Rhodophyta, Bangiophyceae, Bangiales) }\end{array}$ & ORAC: $3.14 \pm 0.32 \mu \mathrm{mol} \mathrm{TE} / \mu \mathrm{M}$ & [200] \\
\hline 234 & $\begin{array}{c}\text { P. dioica } \\
\text { (Rhodophyta, Bangiophyceae, Bangiales) }\end{array}$ & ORAC: $0.09 \pm 0.00 \mu \mathrm{mol} \mathrm{TE} / \mu \mathrm{M}$ & [200] \\
\hline
\end{tabular}


Table 5. Cont.

\begin{tabular}{|c|c|c|c|}
\hline Compound & Isolation Source & Assay/Activity & Reference \\
\hline 235 & $\begin{array}{c}\text { P. dioica } \\
\text { (Rhodophyta, Bangiophyceae, Bangiales) }\end{array}$ & ORAC: $2.85 \pm 0.42 \mu \mathrm{mol} \mathrm{TE} / \mu \mathrm{M}$ & [200] \\
\hline 236 & $\begin{array}{c}\text { P. dioica } \\
\text { (Rhodophyta, Bangiophyceae, Bangiales) }\end{array}$ & ORAC: $2.50 \pm 0.16 \mu \mathrm{mol} \mathrm{TE} / \mu \mathrm{M}$ & [200] \\
\hline 237 & $\begin{array}{c}\text { P. dioica } \\
\text { (Rhodophyta, Bangiophyceae, Bangiales) }\end{array}$ & ORAC: $4.27 \pm 0.15 \mu \mathrm{mol} \mathrm{TE} / \mu \mathrm{M}$ & [200] \\
\hline 238 & $\begin{array}{c}\text { P. dioica } \\
\text { (Rhodophyta, Bangiophyceae, Bangiales) }\end{array}$ & ORAC: $0.92 \pm 0.10 \mu \mathrm{mol} \mathrm{TE} / \mu \mathrm{M}$ & [200] \\
\hline 239 & $\begin{array}{c}\text { Porphyra sp. } \\
\text { (Rhodophyta, Bangiophyceae, Bangiales) }\end{array}$ & $\begin{array}{l}\text { ROO scavenging (CBA): } 0.048 \pm 0.003 \mathrm{mmol} \\
\text { TE } / \mathrm{g}\end{array}$ & [201] \\
\hline 240 & $\begin{array}{c}\text { Enteromorpha prolifera } \\
\text { (Chlorophyta, Ulvophyceae, Ulvales) }\end{array}$ & $\begin{array}{c}\text { DPPH scavenging: } 88.6 \pm 1.3 \% \text { at } 168.7 \mu \mathrm{M} \\
\text { reducing power: } 60 \% \text { at } 843.6 \mu \mathrm{M} \\
\text { ROO scavenging: } 50 \% \text { at } 843.6 \mu \mathrm{M} \\
\text { TPC: } 21.4 \pm 0.1 \mathrm{mg} \mathrm{GAE} / \mathrm{g}\end{array}$ & [202] \\
\hline 241 & $\begin{array}{l}\text { from plants and microalgae, but also from } \\
\text { macroalgae }\end{array}$ & $\begin{array}{c}\beta \text {-carotene bleaching: } 49.63 \% \text { at } 56.0 \mu \mathrm{M} \\
\text { DPPH scavenging: } 13.89 \% \text { at } 56.0 \mu \mathrm{M} \\
\mathrm{Fe}^{2+} \text { chelation: } 55 \% \text { at } 200 \mu \mathrm{M} \\
\text { lipid peroxidation: } 95 \% \text { at } 100 \mu \mathrm{M} \\
\text { ROO scavenging capacity: } 308\end{array}$ & $\begin{array}{l}{[141,203-} \\
205]\end{array}$ \\
\hline 242 & $\begin{array}{c}\text { E. bicyclis } \\
\text { (Ochrophyta, Phaeophyceae, Laminariales) }\end{array}$ & $\begin{array}{c}\text { FTC } \\
\text { TBARS }\end{array}$ & [206] \\
\hline
\end{tabular}

CBA: crocin bleaching activity; DPPH: 1,1-diphenyl-2-picrylhydrazyl free radical; FTC: ferric thiocyanate; GAE: gallic acid equivalents; Nrf2: nuclear factor erythroid 2-related factor 2; ORAC: oxygen radical absorbance capacity; ROO: peroxyl; TBARS: thiobarbituric acid reactive substances; TE: trolox equivalents; TPC: total phenolics content.

Peptides and alkaloids 225-239, isolated from Gloiopeltis furcata, Porphyra sp., and Martensia fragilis, have demonstrated moderate to significant antioxidant activity [91,196-200]. Specifically, mycosporine-like amino acids 225-227 exhibited markedly lower free radical scavenging activities compared to those of ascorbic acid and Trolox [196,197], although heat treatment of porphyra $334(225)$ at temperatures over $100{ }^{\circ} \mathrm{C}$ afforded its dehydrated form (227) and resulted in more than a 100-fold increase in the DPPH radical scavenging activity ( $\mathrm{IC}_{50}=10.1 \mu \mathrm{g} / \mathrm{mL}$ for $227 \mathrm{vs.}>1000 \mu \mathrm{g} / \mathrm{mL}$ for 225) [197].

The histidine-related dipeptides carnosine (228) and anserine (229) were shown to exert comparable antioxidant activities, as measured by ferric thiocyanate and TBARS (85.2\% and $84.1 \%$ inhibition for $\mathbf{2 2 8}$ and $94.4 \%$ and $89.1 \%$ inhibition for $\mathbf{2 2 9}$, respectively), to those of $\alpha$-tocopherol ( $88.2 \%$ and $86.7 \%$, respectively) and BHT (99.8\% and $98.2 \%$, respectively) [198]. Moreover, Cermeno et al. (2019) isolated a series of bioactive peptides (232-238) from Porphyra dioica that displayed significant antioxidant activity as assessed using the ORAC assay [200]. It appears that peptides containing tyrosine in their structure (compounds 232, 233, 235, and 237) possessed higher levels of antioxidant activity.

In an effort to elucidate the mechanism of action of dictyospiromide (231), neuronlike PC12 cells were treated with $\mathrm{H}_{2} \mathrm{O}_{2}$, and its cytoprotective effect against the induced oxidative damage was evaluated [199]. Treatment with dictyospiromide increased cell survival in a dose-dependent manner and reduced $\mathrm{H}_{2} \mathrm{O}_{2}$-induced lactate dehydrogenase ( $\mathrm{LDH}$ ) production at a concentration as low as $0.5 \mu \mathrm{M}$. Additionally, compound 231 was investigated regarding its implication in the Nrf2/ARE signaling pathway, which regulates the expression of genes involved in cellular antioxidant defense. It was found that dictyospiromide (231) exhibited a cytoprotective antioxidant effect in PC12 cells that involved activation of the Nrf2/ARE signaling pathway and enhanced expression of HO-1. 
<smiles>COC1=C(NC(C(=O)O)C(C)O)C=C(CO)CC1=NCC(=O)O</smiles>

225<smiles>[R]C(O)C(N=C1CC(O)(CO)CC(NCC(=O)O)=C1OC)C(=O)O</smiles>

$226 \mathrm{R}=\mathrm{Me}$

$227 \mathrm{R}=\mathrm{H}$<smiles>[R]n1cnc(CC(NC(=O)C(C)N)C(=O)O)c1</smiles>

$228 \mathrm{R}=\mathrm{H}$

$229 \mathrm{R}=\mathrm{Me}$<smiles>CCC(C)CC(C(=O)N/C=C/c1c[nH]c2ccccc12)N(C)Cc1ccc(O)cc1</smiles><smiles>C=C[C@H]1C[C@H](O)[C@@H]([C@@H](C)CCC=C(C)C)[C@]12C(=O)NC(=O)/C2=C/OCC</smiles><smiles>CC(N)C(=O)NCC(=O)N[C@@H](Cc1ccccc1)C(=O)NC(Cc1ccc(O)cc1)C(=O)O</smiles><smiles>CCC(C)C(NC(=O)C(Cc1ccccc1)NC(=O)C(C)N)C(=O)NC(C(=O)O)C(C)O</smiles><smiles>CC(C)CC(NC(=O)C(N)Cc1ccc(O)cc1)C(=O)NC(C(=O)NC(C)C(=O)O)C(C)C</smiles><smiles>CC(C)CC(N)C(=O)NC(CC(=O)O)C(=O)NC(CC(C)C)C(=O)NC(Cc1cc2ccccc2[nH]1)C(=O)O</smiles>

Figure 20. Chemical structures of compounds 225-237. 


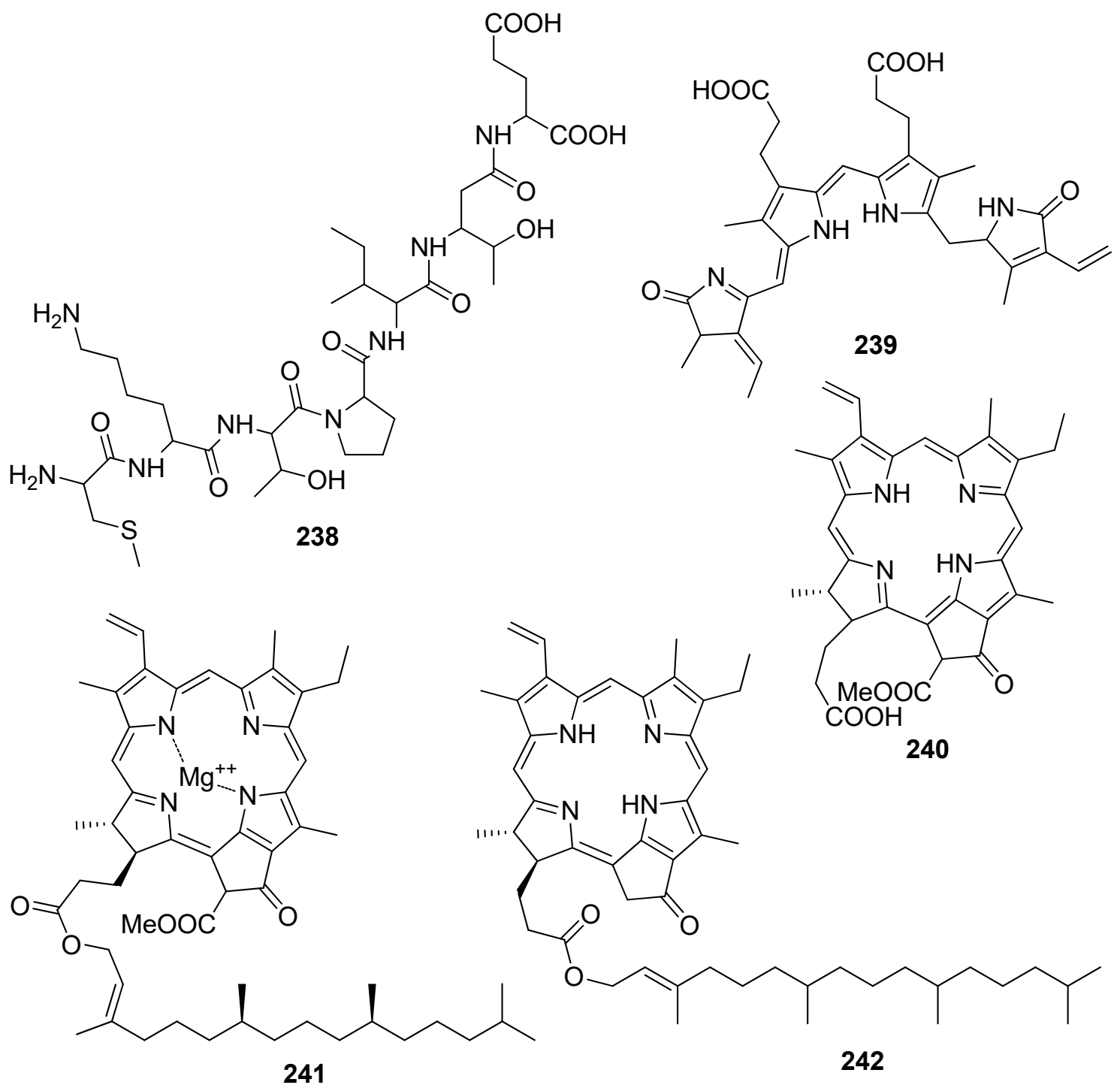

Figure 21. Chemical structures of compounds 238-242.

Chlorophylls are natural pigments with a well-known antioxidant activity. Although their radical scavenging activities are reported to be low [203], their inhibitory action in lipid peroxidation was found to be $95 \%$ at concentrations as low as $100 \mu \mathrm{M}$ [204]. However, knowledge is limited regarding the yield of chlorophyll metabolites, their absorption and transportation processes, their metabolic pathways, and their precise oxidation mechanisms. At the in vitro level, only few researchers have studied the stability of chlorophylls during digestion and subsequent absorption through intestinal cells. The major outcome is that chlorophylls $\alpha$ and $\beta$ are transformed into their corresponding pheophorbides and pheophytins and are absorbed at similar rates to those of carotenoids. Further, it has been shown that pheophorbide a is transported at the intestinal level by a protein-mediated mechanism, with scavenger receptor class B type 1 (SR-BI) being a plausible transporter. These results have been confirmed at the in vivo level, using mice as the experimental model, showing a preferential accumulation of pheophorbide in the liver along with multiple other chlorophyll compounds [205].

The characteristic pigments of the light harvesting proteins phycoerythrobilin (239), pheophorbide a (240), chlorophyll $\beta$ (241) and pyropheophytin $\alpha$ (242) have been found to exert antioxidant activity $[141,201-206]$. It seems that the porphyrin ring system is important for the expression of antioxidative activity in the dark. Indeed, phycoerythrobilin (239) showed potent antioxidant activity in in vitro experiments and significantly inhibited the release of $\beta$-hexosaminidase in rat basophilic leukemia cells [207], suggesting that 
phycoerythrobilin exhibits anti-inflammatory activity. Pheophorbide a (240) demonstrated antioxidant activity (88.6 $\pm 1.3 \%$ DPPH scavenging) higher than that of $\alpha$-tocopherol, and comparable to that of butylated hydroxyanisol (BHA, $85.3 \pm 0.2 \%$ DPPH scavenging) at a concentration of $0.1 \mathrm{mg} / \mathrm{mL}$ [202], while pyropheophytin $\alpha$ (242) demonstrated antioxidant activity higher than that of $\alpha$-tocopherol [206].

\section{Carbohydrates and Polysaccharides}

Carbohydrates ranging in size from simple monosaccharides to high molecular weight polysaccharides isolated from marine macroalgae often exert antioxidant activities $[208,209]$ (Table 6, Figure 22).

Table 6. Carbohydrates and polysaccharides from macroalgae with antioxidant activity.

\begin{tabular}{|c|c|c|c|c|}
\hline Compound & Isolation Source & MW/Sulfate Content & Assay/Activity & Reference \\
\hline 243 & from a plethora of macroalgae & - & $\begin{array}{l}\text { free radicals }\left(\mathrm{DPPH}, \mathrm{OH}, \mathrm{NO}, \mathrm{O}_{2}\right) \\
\text { scavenging } \\
\text { enzyme activity (a-glucosidase, } \mathrm{AChE}, \\
\text { BChE) }\end{array}$ & [210] \\
\hline 244 & $\begin{array}{c}\text { Laurencia undulata } \\
\text { (Rhodophyta, Florideophyceae, } \\
\text { Ceramiales) }\end{array}$ & - & $\begin{array}{c}\text { alkyl scavenging: } \mathrm{IC}_{50}=43.7 \mu \mathrm{M} \\
\text { DPPH scavenging: } \mathrm{IC}_{50}=39.3 \mu \mathrm{M} \\
\text { OH scavenging: } \mathrm{IC}_{50}=27.4 \mu \mathrm{M} \\
\mathrm{O}_{2}{ }^{-} \text {scavenging: } \mathrm{IC}_{50}=39.4 \mu \mathrm{M} \\
\text { gene expression levels of } \mathrm{GSH} \text { and } \\
\text { SOD } \\
\text { intracellular ROS levels (DCFH-DA) } \\
\text { in RAW264.7 cells } \\
\text { membrane protein oxidation } \\
\text { MPO activity } \\
\text { protein expression of MMP2 and } \\
\text { MMP9 }\end{array}$ & [211] \\
\hline 245 & $\begin{array}{c}\text { L. undulata } \\
\text { (Rhodophyta, Florideophyceae, } \\
\text { Ceramiales) }\end{array}$ & - & $\begin{array}{c}\text { alkyl scavenging: } \mathrm{IC}_{50}=32.3 \mu \mathrm{M} \\
\text { DPPH scavenging: } \mathrm{IC}_{50}=41.8 \mu \mathrm{M} \\
\mathrm{OH} \text { scavenging: } \mathrm{IC}_{50}=22.7 \mu \mathrm{M} \\
\mathrm{O}_{2}{ }^{-} \text {scavenging: } \mathrm{IC}_{50}=33.6 \mu \mathrm{M} \\
\text { gene expression levels of GSH and } \\
\text { SOD } \\
\text { intracellular ROS levels (DCFH-DA) } \\
\text { in RAW264.7 cells } \\
\text { membrane protein oxidation } \\
\text { MPO activity } \\
\text { protein expression of MMP2 and } \\
\text { MMP9 }\end{array}$ & [211] \\
\hline 246 & $\begin{array}{l}\text { enzymatically produced from } \\
\text { commercially available } \\
\text { polysaccharides }\end{array}$ & n.d. & $\begin{array}{c}\mathrm{OH} \text { scavenging } \\
\mathrm{O}_{2}^{-} \text {scavenging } \\
\text { erythrocyte hemolysis inhibiting } \\
\text { lipid peroxidation } \\
\text { metal chelating activity }\end{array}$ & [212] \\
\hline 247 & $\begin{array}{l}\text { enzymatically produced from } \\
\text { commercially available } \\
\text { polysaccharides }\end{array}$ & n.d. & $\begin{array}{c}\text { OH scavenging } \\
\mathrm{O}_{2}^{-} \text {scavenging } \\
\text { erythrocyte hemolysis inhibiting } \\
\text { lipid peroxidation } \\
\text { metal chelating activity }\end{array}$ & [212] \\
\hline
\end{tabular}


Table 6. Cont.

\begin{tabular}{|c|c|c|c|c|}
\hline Compound & Isolation Source & MW/Sulfate Content & Assay/Activity & Reference \\
\hline 248 & $\begin{array}{c}\text { F. vesiculosus } \\
\text { (Ochrophyta, Phaeophyceae, } \\
\text { Fucales) }\end{array}$ & $170 \mathrm{kDa} / 44.10 \pm 0.16 \%$ & $\begin{array}{c}\text { OH scavenging: } \\
\mathrm{IC}_{50}=0.157 \pm 0.005 \mathrm{mg} / \mathrm{mL} \\
\mathrm{O}_{2}{ }^{-} \text {scavenging: } \\
\mathrm{IC}_{50}=0.058 \pm 0.011 \mathrm{mg} / \mathrm{mL} \\
\text { liver microsomal lipid peroxidation: } \\
\mathrm{IC}_{50}=1.250 \pm 0.174 \mathrm{mg} / \mathrm{mL}\end{array}$ & [213] \\
\hline 249 & $\begin{array}{c}\text { Cystoseira sedoides } \\
\text { (Ochrophyta, Phaeophyceae, } \\
\text { Fucales) }\end{array}$ & $642 \mathrm{kDa} / 16.3 \%$ & $\begin{array}{c}\text { DPPH scavenging: } \\
\mathrm{IC}_{50}=0.96 \pm 0.01 \mathrm{mg} / \mathrm{mL}\end{array}$ & [214] \\
\hline 250 & $\begin{array}{c}\text { Cystoseira compressa } \\
\text { (Ochrophyta, Phaeophyceae, } \\
\text { Fucales) }\end{array}$ & $545 \mathrm{kDa} / 16.6 \%$ & $\begin{array}{c}\text { DPPH scavenging: } \\
\mathrm{IC}_{50}=0.84 \pm 0.06 \mathrm{mg} / \mathrm{mL}\end{array}$ & [214] \\
\hline 251 & $\begin{array}{c}\text { C. crinita } \\
\text { (Ochrophyta, Phaeophyceae, } \\
\text { Fucales) }\end{array}$ & $339 \mathrm{kDa} / 15.7 \%$ & $\begin{array}{c}\text { DPPH scavenging: } \\
\mathrm{IC}_{50}=0.76 \pm 0.04 \mathrm{mg} / \mathrm{mL}\end{array}$ & [214] \\
\hline 252 & $\begin{array}{c}\text { Padina gymnospora } \\
\text { (Ochrophyta, Phaeophyceae, } \\
\text { Dictyotales) }\end{array}$ & $200 \mathrm{kDa} / 18.40 \pm 0.28 \%$ & $\begin{array}{c}\text { OH scavenging } \\
\mathrm{O}_{2}^{-} \text {scavenging: } \\
\mathrm{IC}_{50}=0.243 \pm 0.014 \mathrm{mg} / \mathrm{mL} \\
\text { liver microsomal lipid peroxidation: } \\
\mathrm{IC}_{50}=2.753 \pm 0.051 \mathrm{mg} / \mathrm{mL}\end{array}$ & [213] \\
\hline 253 & $\begin{array}{c}\text { P. gymnospora } \\
\text { (Ochrophyta, Phaeophyceae, } \\
\text { Dictyotales) }\end{array}$ & $18 \mathrm{kDa} / 27.57 \pm 0.17 \%$ & $\begin{array}{c}\text { OH scavenging: } \\
\mathrm{IC}_{50}=0.353 \pm 0.036 \mathrm{mg} / \mathrm{mL} \\
\mathrm{O}_{2}{ }^{-} \text {scavenging: } \\
\mathrm{IC}_{50}=0.243 \pm 0.013 \mathrm{mg} / \mathrm{mL} \\
\text { liver microsomal lipid peroxidation: } \\
\mathrm{IC}_{50}=23.887 \pm 5.975 \mathrm{mg} / \mathrm{mL}\end{array}$ & [213] \\
\hline 254 & $\begin{array}{c}\text { L. japonica } \\
\text { (Ochrophyta, Phaeophyceae, } \\
\text { Laminariales) }\end{array}$ & $742 \mathrm{kDa} / 16.5 \%$ & $\begin{array}{l}\text { OH scavenging: } \mathrm{IC}_{50}=0.60 \mathrm{mg} / \mathrm{mL} \\
\mathrm{O}_{2}{ }^{-} \text {scavenging: } \mathrm{IC}_{50}=0.43 \mathrm{mg} / \mathrm{mL}\end{array}$ & [215] \\
\hline 255 & $\begin{array}{c}\text { L. japonica } \\
\text { (Ochrophyta, Phaeophyceae, } \\
\text { Laminariales) }\end{array}$ & $175.9 \mathrm{kDa} / 33.5 \%$ & $\begin{array}{l}\text { OH scavenging: } \mathrm{IC}_{50}=0.85 \mathrm{mg} / \mathrm{mL} \\
\mathrm{O}_{2}{ }^{-} \text {scavenging: } \mathrm{IC}_{50}=0.53 \mathrm{mg} / \mathrm{mL}\end{array}$ & [215] \\
\hline 256 & $\begin{array}{c}\text { Undaria pinnatifida } \\
\text { (Ochrophyta, Phaeophyceae, } \\
\text { Laminariales) }\end{array}$ & $10 \mathrm{kDa} / \mathrm{n} . \mathrm{d}$ & $\begin{array}{l}\text { DPPH scavenging: } 8.77 \pm 1.24 \mathrm{TE} \\
\text { ( } \mu \mathrm{g} / \mathrm{mL}) \\
\text { OH scavenging: } 86.98 \pm 1.16 \%\end{array}$ & [216] \\
\hline 257 & $\begin{array}{c}\text { U. pinnatifida } \\
\text { (Ochrophyta, Phaeophyceae, } \\
\text { Laminariales) }\end{array}$ & $300 \mathrm{kDa} / 20.01 \pm 0.82 \%$ & $\begin{array}{l}\text { DPPH scavenging: } 9.01 \pm 1.93 \mathrm{TE} \\
\text { ( } \mu \mathrm{g} / \mathrm{mL}) \\
\text { OH scavenging: } 74.32 \pm 1.41 \%\end{array}$ & [216] \\
\hline 258 & $\begin{array}{c}\text { F. vesiculosus } \\
\text { (Ochrophyta, Phaeophyceae, } \\
\text { Fucales) }\end{array}$ & n.d. $/ 21.1 \pm 1.7 \%$ & $\begin{array}{c}\text { ABTS }^{+} \text {scavenging } \\
\text { DPPH scavenging } \\
\text { lipid oxidation } \\
\text { differential pulse voltammetry }\end{array}$ & {$[217]$} \\
\hline 259 & $\begin{array}{c}\text { F. vesiculosus } \\
\text { (Ochrophyta, Phaeophyceae, } \\
\text { Fucales) }\end{array}$ & n.d. $/ 21.2 \pm 0.8 \%$ & $\begin{array}{c}\text { ABTS }^{+} \text {scavenging } \\
\text { DPPH scavenging } \\
\text { lipid oxidation } \\
\text { differential pulse voltammetry }\end{array}$ & [217] \\
\hline 260 & $\begin{array}{c}\text { F. vesiculosus } \\
\text { (Ochrophyta, Phaeophyceae, } \\
\text { Fucales) }\end{array}$ & n.d. $/ 27.0 \%$ & $\begin{array}{c}\text { DPPH scavenging: } \\
\mathrm{IC}_{50}=0.035 \pm 0.002 \mathrm{mg} / \mathrm{mL} \\
\text { reducing power: } \\
\mathrm{RC}_{0.5 \mathrm{AU}}=1.48 \mathrm{mg} / \mathrm{mL}\end{array}$ & [218] \\
\hline
\end{tabular}


Table 6. Cont.

\begin{tabular}{|c|c|c|c|c|}
\hline Compound & Isolation Source & MW/Sulfate Content & Assay/Activity & Reference \\
\hline 261 & $\begin{array}{c}\text { Sargassum binderi } \\
\text { (Ochrophyta, Phaeophyceae, } \\
\text { Fucales) }\end{array}$ & n.d./n.d. & $\begin{array}{c}\text { DPPH scavenging: } \\
\mathrm{IC}_{50}=2.01 \pm 0.29 \mathrm{mg} / \mathrm{mL} \\
\mathrm{OH} \text { scavenging: } 60.95 \pm 0.69 \% \\
\mathrm{O}_{2}{ }^{-} \text {scavenging: } 26.78 \pm 1.90 \% \\
\text { reducing power: } 0.60 \pm 0.08 \mathrm{mg} \\
\text { GAE } 100 \mathrm{~g}\end{array}$ & [219] \\
\hline 262 & $\begin{array}{l}\text { hydrolyzed from commercially } \\
\text { available polysaccharides }\end{array}$ & 5-30 kDa/n.d. & $\begin{array}{l}\text { LPS-induced ROS generation in RAW } \\
264.7 \text { macrophages }\end{array}$ & [220] \\
\hline 263 & not specified & n.d./n.d. & $\begin{array}{l}\text { HO-1, SOD1, Nrf2 and } \\
\text { Keap1 expression in human } \\
\text { keratinocytes }\end{array}$ & [221] \\
\hline 264 & $\begin{array}{c}\text { U. pinnatifida } \\
\text { (Ochrophyta, Phaeophyceae, } \\
\text { Laminariales) }\end{array}$ & n.d./n.d. & $\begin{array}{c}\text { DPPH scavenging } \\
\text { metal chelating activity } \\
\text { NO scavenging } \\
\text { OH scavenging } \\
\text { reducing power } \\
\text { arthritis-induced physical changes in } \\
\text { rats }\end{array}$ & [222] \\
\hline 265 & $\begin{array}{c}\text { Eucheuma spinosa } \\
\text { (Rhodophyta, Florideophyceae, } \\
\text { Gigartinales) }\end{array}$ & n.d. $/ 27.60 \pm 0.12 \%$ & $\begin{array}{c}\text { OH scavenging: } \\
\mathrm{IC}_{50}=0.281 \pm 0.072 \mathrm{mg} / \mathrm{mL} \\
\mathrm{O}_{2}^{-} \text {scavenging: } \\
\mathrm{IC}_{50}=0.332 \pm 0.080 \mathrm{mg} / \mathrm{mL} \\
\text { liver microsomal lipid peroxidation: } \\
\mathrm{IC}_{50}=0.830 \pm 0.063 \mathrm{mg} / \mathrm{mL}\end{array}$ & [213] \\
\hline 266 & $\begin{array}{c}\text { Eucheuma cottonii } \\
\text { (Rhodophyta, Florideophyceae, } \\
\text { Gigartinales) }\end{array}$ & n.d. $/ 17.90 \pm 0.05 \%$ & $\begin{array}{c}\text { OH scavenging: } \\
\mathrm{IC}_{50}=0.335 \pm 0.016 \mathrm{mg} / \mathrm{mL} \\
\mathrm{O}_{2}-\text { scavenging: } \\
\mathrm{IC}_{50}=0.112 \pm 0.003 \mathrm{mg} / \mathrm{mL} \\
\text { liver microsomal lipid peroxidation: } \\
\mathrm{IC}_{50}=0.323 \pm 0.011 \mathrm{mg} / \mathrm{mL}\end{array}$ & [213] \\
\hline 267 & $\begin{array}{c}\text { Gigartina acicularis, Gigartina } \\
\text { pisillata } \\
\text { (Rhodophyta, Florideophyceae, } \\
\text { Gigartinales) }\end{array}$ & n.d. $/ 33.38 \pm 0.06 \%$ & $\begin{array}{c}\text { OH scavenging: } \\
\mathrm{IC}_{50}=0.357 \pm 0.120 \mathrm{mg} / \mathrm{mL} \\
\mathrm{O}_{2}^{-} \text {scavenging: } \\
\mathrm{IC}_{50}=0.046 \pm 0.001 \mathrm{mg} / \mathrm{mL} \\
\text { liver microsomal lipid peroxidation: } \\
\mathrm{IC}_{50}=2.697 \pm 0.267 \mathrm{mg} / \mathrm{mL}\end{array}$ & [213] \\
\hline 268 & $\begin{array}{c}\text { Porphyra haitanensis } \\
\text { (Rhodophyta, Bangiophyceae, } \\
\text { Bangiales) }\end{array}$ & n.d. $/ 17.7 \%$ & $\begin{array}{l}\text { OH scavenging: } \mathrm{IC}_{50}=6.55 \mathrm{mg} / \mathrm{mL} \\
\mathrm{O}_{2}{ }^{-} \text {scavenging: } \sim 60 \% \text { at } 2.5 \mu \mathrm{g} / \mathrm{mL} \\
\text { reducing power: } 0.42 \text { at } 6.17 \mathrm{mg} / \mathrm{mL}\end{array}$ & [223] \\
\hline 269 & $\begin{array}{c}\text { Ulva pertusa } \\
\text { (Chlorophyta, Ulvophyceae, } \\
\text { Ulvales) }\end{array}$ & n.d./19.5\% & $\begin{array}{c}\text { OH scavenging } \\
\mathrm{O}_{2}^{-} \text {scavenging: } \mathrm{IC}_{50}=20.0 \mu \mathrm{g} / \mathrm{mL} \\
\text { metal chelating assay } \\
\text { reducing power }\end{array}$ & [224] \\
\hline 270 & $\begin{array}{c}\text { U. pertusa } \\
\text { (Chlorophyta, Ulvophyceae, } \\
\text { Ulvales) }\end{array}$ & $151.7 \mathrm{kDa} / \mathrm{n} . \mathrm{d}$. & $\begin{array}{c}\mathrm{Fe}^{2+} \text { chelation } \\
\mathrm{OH} \text { scavenging: } \mathrm{IC}_{50}>1 \mathrm{mg} / \mathrm{mL} \\
\mathrm{O}_{2}^{-} \text {scavenging: } \mathrm{IC}_{50}=22.1 \mu \mathrm{g} / \mathrm{mL} \\
\text { reducing power }\end{array}$ & [225] \\
\hline
\end{tabular}


Table 6. Cont.

\begin{tabular}{|c|c|c|c|c|}
\hline Compound & Isolation Source & MW/Sulfate Content & Assay/Activity & Reference \\
\hline 271 & $\begin{array}{c}\text { U. pertusa } \\
\text { (Chlorophyta, Ulvophyceae, } \\
\text { Ulvales) }\end{array}$ & n.d./n.d. & $\begin{array}{c}\mathrm{Fe}^{2+} \text { chelation: } 10 \% \text { to } 20 \% \text { at } \\
0.31-1.88 \mathrm{mg} / \mathrm{mL} \\
\text { OH scavenging: } 3.3-37 \% \text { at } \\
0.25-1.52 \mathrm{mg} / \mathrm{mL} \\
\mathrm{O}_{2}{ }^{-} \text {scavenging: } \mathrm{IC}_{50}=9.17 \mu \mathrm{g} / \mathrm{mL} \\
\text { reducing power }\end{array}$ & [226] \\
\hline
\end{tabular}

AChE: acetylcholinesterase; BChE: butyrylcholinesterase; DPPH: 1,1-diphenyl-2-picrylhydrazyl free radical; GAE: gallic acid equivalents; GSH: glutathione; HO-1: heme oxygenase-1; LPS: lipopolysaccharide; MMP: matrix metalloproteinase; MPO: myeloperoxidase; n.d.: not determined; NO: nitric oxide; Nrf2: nuclear factor erythroid 2-related factor 2; $\mathrm{OH}$ : hydroxyl; $\mathrm{O}_{2}{ }^{-}$: superoxide anion; $\mathrm{RC}_{0.5 \mathrm{AU}}$ : reducing capacity at 0.5 absorbance unit; ROS: reactive oxygen species; SOD: superoxide dismutase; TE: trolox equivalents.

The simplest sugar alcohol isolated from a plethora of macroalgae is mannitol (243), representing up to $9 \%, 47 \%$, and $59 \%$ of the dry algal weight in Chlorophyta, Rhodophyta and Ochrophyta, respectively [210]. Antioxidant activity evaluation by enzymes $(\alpha-$ glucosidase, acetyl (AChE) and butyrylcholinesterase (BuChE)) and free radicals (DPPH, $\mathrm{NO}, \mathrm{OH}$, and $\mathrm{O}_{2}{ }^{-}$) revealed that higher contents of mannitol are closely related with cholinesterases and DPPH radical scavenging, and to a lesser extent are responsible for $\alpha$-glucosidase inhibition, $\mathrm{OH}, \mathrm{O}_{2}{ }^{-}$, and $\mathrm{NO}$ scavenging.

Two simple glucosides, floridoside (244) and D-isofloridoside (245), have been isolated from the red alga Laurencia undulata and their free radical scavenging activity, inhibition of intracellular ROS levels, the level of membrane protein oxidation, myeloperoxidase (MPO) activity inhibition, gene expression levels of GSH and SOD, and protein expression of MMP2 and MMP9 have been determined [211]. It was found that both floridoside (244) and D-isofloridoside (245) possess significant antioxidant capacity and are potential inhibitors of MMP2 and MMP9.

Marine macroalgae are the most important source of non-animal sulfated polysaccharides (SPs), with the main categories being fucoidans isolated from brown algae, carrageenans and porphyrans isolated from red algae and ulvans isolated from green algae. SPs possess excellent in vitro antioxidant activity, including both radical scavenging capacity and metal chelating ability $[212,227,228]$. The antioxidant activity of SPs directly related to their structural features, such as degree of sulfation, molecular weight (MW), type of major sugar, and glycosidic branching [212,225,229]. For example, low MW SPs have shown potent antioxidant activity, stronger than that of high MW SPs [230]. The rationale for this is that low MW SPs may be incorporated into the cells more efficiently and donate proton effectively compared to high MW SPs.

Alginate oligosaccharide $(\mathrm{AO}, 246)$ and fucoidan oligosaccharide $(\mathrm{FO}, 247)$ were enzymatically produced from commercially available polysaccharides and their antioxidant activity was studied [212]. AO (246) had the highest hydroxyl radical scavenging activity as compared to FO (247), while in the $\mathrm{Fe}^{2+}$ chelation assay, FO exhibited good chelation in contrast to $\mathrm{AO}$ that hardly displayed any activity.

Fucoidans of diverse MW and sulfation degree (247-264) have been isolated from various brown algae and/or chemically modified and their antioxidant activity has been tested employing $\mathrm{OH}$ and $\mathrm{O}_{2}{ }^{-}$scavenging, erythrocyte hemolysis inhibition, metal chelation, and anti-lipid peroxidation assays [212-215]. In the study of Zhao et al. (2008) two fractions of different MW, namely $742 \mathrm{kDa}$ (254) and $175.9 \mathrm{kDa}$ (255), were obtained from fucoidans extracted from L. japonica and evaluated for their $\mathrm{OH}$ and $\mathrm{O}_{2}{ }^{-}$scavenging activity, with the higher MW fraction exhibiting higher levels of activity [215]. Following radical process degradation, an ascophyllan-like fraction rich in glucuronic acid and a fraction rich in galactose and mannose were confirmed as responsible for the oxygen free radical scavenging activity [215]. On the contrary, Koh et al. (2019) reported on the higher antioxidant capacity of low MW (10 kDa) fucoidan (256) from Undaria pinnatifida (close to that of BHA) as compared to a high MW (300 kDa) fucoidan (257) [216]. 
<smiles>OC[C@@H](O)[C@@H](O)[C@H](O)[C@H](O)CO</smiles>

243

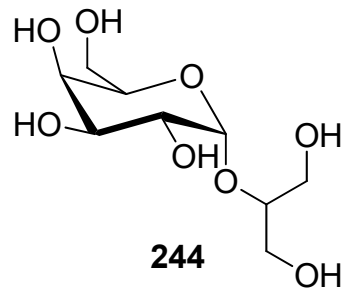

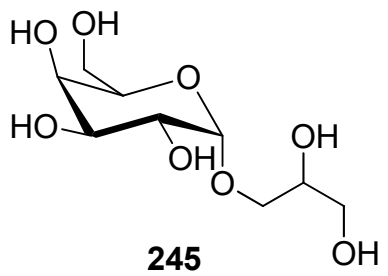

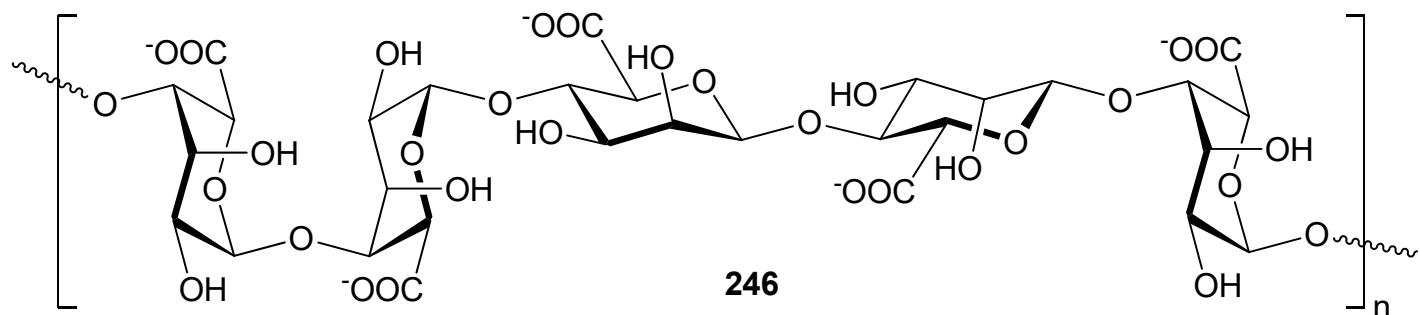

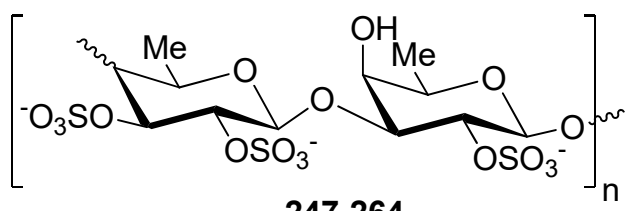

247-264

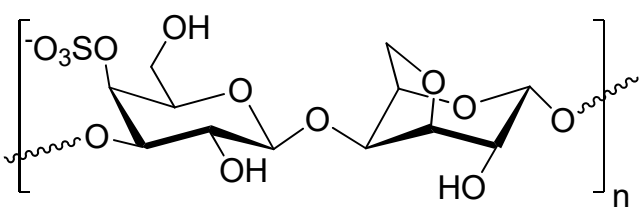

266

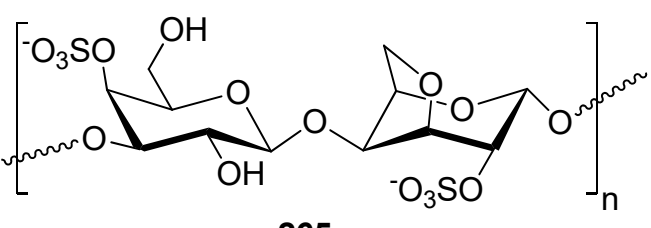

265

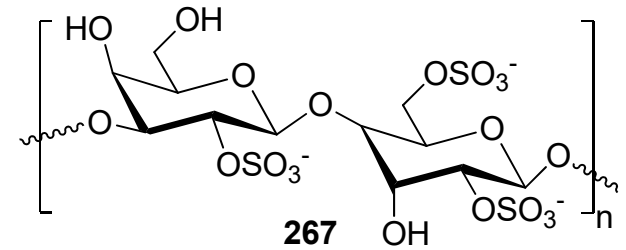

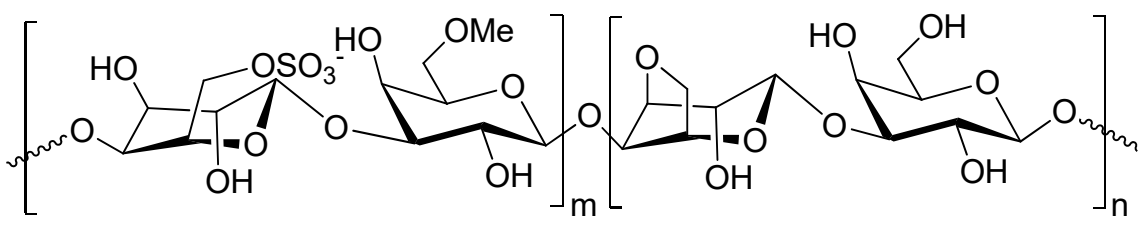

268

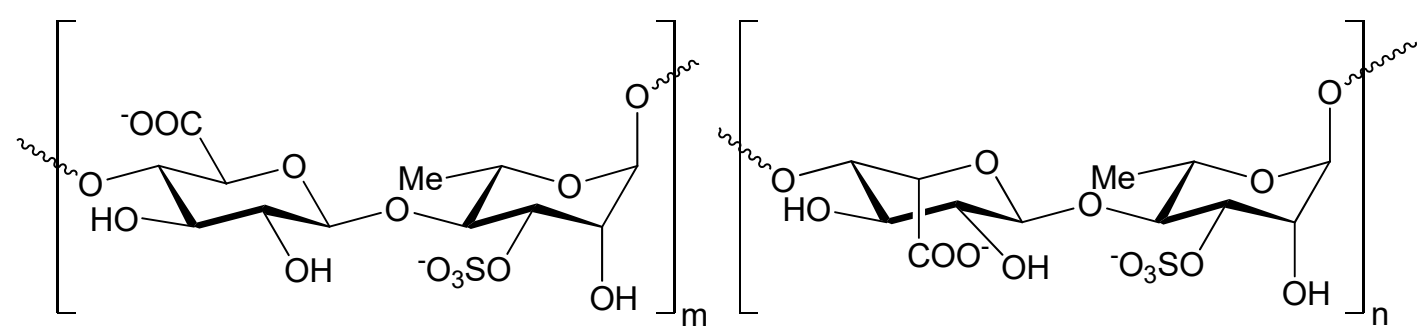

269-271

Figure 22. Chemical structures of compounds 243-271.

Additionally, Rodriguez-Jasso et al. (2014) isolated fucose-containing sulfated polysaccharides from Fucus vesiculosus using either microwave-assisted extraction (258) or autohydrolysis (259) and their antioxidant activity was determined [217]. Both samples presented similar sulfate contents $(\sim 21 \%)$, as well as comparable antioxidant potential as evaluated by $\mathrm{DPPH}$ and $\mathrm{ABTS}^{+}$scavenging, and lipid oxidation inhibition methods. Differences in the antioxidant potential could be observed only when using a differential pulse voltammetry 
technique, pointing to structural variations of the fucans obtained by the two different methods.

Several studies have reported the in vitro and in vivo antioxidant efficacy of fucoidan [231]. Kim et al. (2012) have demonstrated that low MW fucoidan (262) might block NO, as well as ROS production, suppressing therefore oxidative stress and MAPKs in RAW264.7 cells [220]. Additionally, fucoidan (263) was found to reduce the oxidative stress through Nrf2/ERK signaling mediated regulation of HO-1 and SOD1 expression in human keratinocytes [221]. More recently, Phull et al. (2017) have demonstrated that fucoidans derived from $U$. pinnatifida (264) exhibit significant in vitro and in vivo anti-arthritic responses in rabbit articular chondrocytes and rats, respectively. Moreover, administration of fucoidan to arthritic rats ameliorated the clinical symptoms and led to the overall improvement of their health [222].

Rocha de Souza et al. (2007) reported on the isolation of iota ( ( )- (265), kappa (K)- (266), and lambda $(\lambda)$ - (267) carrageenans from various red algae and their antioxidant activity as evaluated by the scavenging of $\mathrm{OH}$ and $\mathrm{O}_{2}{ }^{-}$radicals, and lipid peroxidation assays [213]. The results of the study indicated that, among the different carrageenans, $\lambda$-carrageenan (267) exhibited the highest antioxidant and free radical scavenging activity. Thus, a positive correlation between sulfate content and antioxidant activity was evidenced.

Acetylation, phosphorylation and benzoylation of porphyran (268) extracted from the red alga Porphyra haitanensis afforded derivatives with improved antioxidant activity, as evaluated in superoxide radical, hydroxyl radical and reducing power assays [223]. In a previous study, Zhang et al. (2003) obtained through anion-exchange column chromatography three sulfated polysaccharide fractions with variable sulfate content $(17.4 \%, 20.5 \%$ and $33.5 \%$ ) from the same red algal species and investigated their in vitro antioxidant activities [229]. All three showed strong scavenging effect on superoxide radical and much weaker effect on hydroxyl free radical, while lipid peroxide in the rat liver microsome was significantly inhibited. In two subsequent studies the fractions with sulfate contents $17.4 \%$ and $20.5 \%$ were evaluated in vivo in aging mice $[48,49]$. In both cases, intraperitoneal administration significantly decreased lipid peroxidation in a dose-dependent manner, while at the same time increasing total antioxidant capacity and the activity of SOD and GPx in all organs of the aging mice.

Ulvans of diverse sulfation degree and MW (269-271) have been isolated from the green alga Ulva pertusa and/or chemically modified and their antioxidant activity was tested employing $\mathrm{OH}$ and $\mathrm{O}_{2}{ }^{-}$radical scavenging, reducing power and metal chelating assays [224-226]. Specifically, Qi et al. (2005) extracted ulvan (269) with 19.5\% sulfate content and chemically prepared derivatives of higher sulfate content ranging from $23.5 \%$ to $32.8 \%$. Upon evaluation of their $\mathrm{O}_{2}{ }^{-}$radical and $\mathrm{OH}$ radical scavenging activity, it was observed that the derivatives displayed higher levels of activity, ranging from $91.7 \%$ to $95.5 \%$ at a concentration as low as $23.0 \mu \mathrm{g} / \mathrm{mL}$ for $\mathrm{O}_{2}{ }^{-}$radical scavenging and with $\mathrm{IC}_{50}$ values ranging from 0.46 to $1.43 \mathrm{mg} / \mathrm{mL}$ for $\mathrm{OH}$ radical scavenging [224].

In another study, Qi et al. (2005) initially extracted ulvan (270) from U. pertusa, and subsequently, three derivatives of different $\mathrm{MW}$ were prepared by $\mathrm{H}_{2} \mathrm{O}_{2}$ degradation and their antioxidant activities, including $\mathrm{OH}$ and $\mathrm{O}_{2}{ }^{-}$radical scavenging activity, reducing power and metal chelating ability, were investigated [225]. The MW of the natural and degraded ulvans were calculated at 151.7, 28.2, 58.0, and 64.5, kDa, respectively. All polysaccharides exhibited significant $\mathrm{OH}$ and $\mathrm{O}_{2}{ }^{-}$radical scavenging capacity at all concentrations tested with similar $\mathrm{IC}_{50}$ values at about $>1 \mathrm{mg} / \mathrm{mL}$ and $22.1 \mu \mathrm{g} / \mathrm{mL}$, respectively. Among the natural ulvan and the obtained derivatives, the lowest MW one showed the strongest reducing power and metal chelating ability. The results indicated that MW had a significant effect on the antioxidant activity of ulvan, with low MW ulvan exerting the strongest antioxidant activity. In a further study, Qi et al. (2006) prepared derivatives of ulvan (262) after acetylation and benzoylation, which exhibited higher levels of antioxidant activity, as determined using in vitro assays, including scavenging activity against superoxide and hydroxyl radicals, reducing power, and chelating ability [226]. 


\section{Miscellaneous Compounds}

A number of compounds (272-301, Figures 23 and 24) isolated from marine macroalgae, displaying various structures that do not belong to the previously described classes, have also exhibited levels of antioxidant activity worth mentioning (Table 7).

Table 7. Miscellaneous compounds from macroalgae with antioxidant activity.

\begin{tabular}{|c|c|c|c|}
\hline Compound & Isolation Source & Assay/Activity & Reference \\
\hline 272 & $\begin{array}{c}\text { G. furcata } \\
\text { (Rhodophyta, Florideophyceae, Gigartinales) }\end{array}$ & $\begin{array}{c}\text { DPPH scavenging: } \mathrm{IC}_{50}=290.5 \pm 1.5 \mu \mathrm{M} \\
\text { ONOO }^{-} \text {scavenging: } \mathrm{IC}_{50}=8.45 \pm 0.46 \mu \mathrm{M} \\
\text { AChE inhibition: } \mathrm{IC}_{50}=94.4 \pm 1.7 \mu \mathrm{M} \\
\text { BChE inhibition: } \mathrm{IC}_{50}=242.0 \pm 4.8 \mu \mathrm{M}\end{array}$ & [75] \\
\hline 273 & $\begin{array}{c}\text { G. furcata } \\
\text { (Rhodophyta, Florideophyceae, Gigartinales) }\end{array}$ & 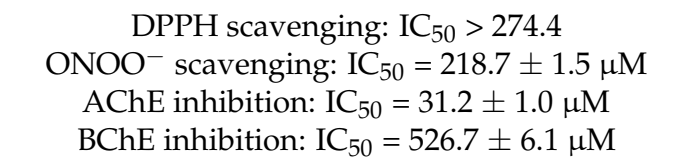 & [75] \\
\hline 274 & $\begin{array}{c}\text { G. furcata } \\
\text { (Rhodophyta, Florideophyceae, Gigartinales) }\end{array}$ & 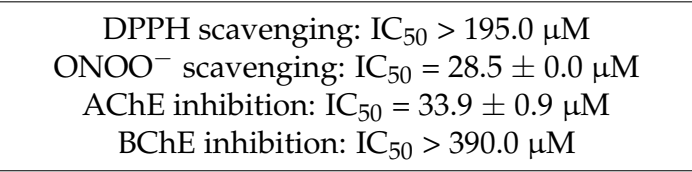 & [75] \\
\hline 275 & $\begin{array}{c}\text { Cystoseira sp. } \\
\text { (Ochrophyta, Phaeophyceae, Fucales) }\end{array}$ & $\begin{array}{c}\text { guglone-induced oxidative stress and } \\
\text { intracellular ROS measurement in Caenorhabditis } \\
\text { elegans }\end{array}$ & {$[141,232]$} \\
\hline 276 & $\begin{array}{c}\text { G. furcata } \\
\text { (Rhodophyta, Florideophyceae, Gigartinales) }\end{array}$ & $\begin{array}{c}\text { DPPH scavenging: } \mathrm{IC}_{50}>179.6 \mu \mathrm{M} \\
\text { ONOO }{ }^{-} \text {scavenging: } \mathrm{IC}_{50}=58.3 \pm 0.3 \mu \mathrm{M} \\
\text { AChE inhibition: } \mathrm{IC}_{50}=44.9 \pm 1.4 \mu \mathrm{M} \\
\text { BChE inhibition: } \mathrm{IC}_{50}=57.1 \pm 2.7 \mu \mathrm{M}\end{array}$ & [75] \\
\hline 277 & $\begin{array}{c}\text { G. furcata } \\
\text { (Rhodophyta, Florideophyceae, Gigartinales) }\end{array}$ & 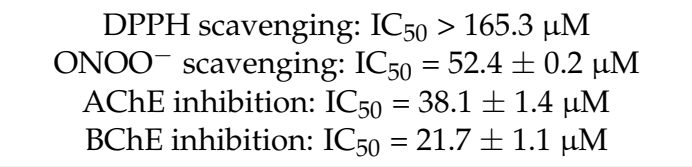 & [75] \\
\hline 278 & $\begin{array}{c}\text { L. undulata } \\
\text { (Rhodophyta, Florideophyceae, Ceramiales) }\end{array}$ & $\begin{array}{c}\text { alkyl scavenging: } \mathrm{IC}_{50}=45.0 \pm 1.6 \mu \mathrm{M} \\
\text { DPPH scavenging: } \mathrm{IC}_{50}=27.1 \pm 1.1 \mu \mathrm{M} \\
\mathrm{OH} \text { scavenging: } \mathrm{IC}_{50}=22.8 \pm 0.8 \mu \mathrm{M} \\
\mathrm{O}_{2}{ }^{-} \text {scavenging: } \mathrm{IC}_{50}=33.5 \pm 1.3 \mu \mathrm{M} \\
\text { gene expression of enzymes GSH and SOD } \\
\text { intracellular ROS levels (DCFH-DA) in } \\
\text { RAW264.7 cells } \\
\text { membrane protein oxidation } \\
\text { MPO activity }\end{array}$ & [233] \\
\hline
\end{tabular}

$279 \quad$ G. furcata

(Rhodophyta, Florideophyceae, Gigartinales)
DPPH scavenging: $\mathrm{IC}_{50}>220.9 \mu \mathrm{M}$

$\mathrm{ONOO}^{-}$scavenging: $\mathrm{IC}_{50}=206.6 \pm 1.0 \mu \mathrm{M}$

AChE inhibition: $\mathrm{IC}_{50}=13.6 \pm 0.5 \mu \mathrm{M}$

BChE inhibition: $\mathrm{IC}_{50}=420.1 \pm 7.8 \mu \mathrm{M}$

ABTS ${ }^{+}$scavenging: $\mathrm{IC}_{50}=3.63 \pm 0.55 \mathrm{mM}$

Kappaphycus alvarezii

DPPH scavenging: $\mathrm{IC}_{50}=3.53 \pm 0.05 \mathrm{mM}$

(Rhodophyta, Florideophyceae, Gigartinales)

ABTS ${ }^{+}$scavenging: $\mathrm{IC}_{50}=1.96 \pm 0.51 \mathrm{mM}$

K. alvarezii

(Rhodophyta, Florideophyceae, Gigartinales)

DPPH scavenging: $\mathrm{IC}_{50}=1.75 \pm 0.20 \mathrm{mM}$

Jania rubens

ABTS $^{+}$scavenging: $\mathrm{IC}_{50}=1.48 \mathrm{mM}$

(Rhodophyta, Florideophyceae, Corallinales) 
Table 7. Cont.

\begin{tabular}{|c|c|c|c|}
\hline Compound & Isolation Source & Assay/Activity & Reference \\
\hline 283 & $\begin{array}{c}\text { S. wightii } \\
\text { (Ochrophyta, Phaeophyceae, Fucales) }\end{array}$ & $\begin{array}{c}\text { ABTS }{ }^{+} \text {scavenging: } \mathrm{IC}_{50}=2.89 \pm 0.04 \mathrm{mM} \\
\text { DPPH scavenging: } \mathrm{IC}_{50}=2.44 \pm 0.11 \mathrm{mM} \\
\mathrm{Fe}^{2+} \text { chelation: } \mathrm{IC}_{50}=3.64 \pm 0.08 \mathrm{mM}\end{array}$ & [236] \\
\hline 284 & $\begin{array}{c}\text { S. wightii } \\
\text { (Ochrophyta, Phaeophyceae, Fucales) }\end{array}$ & $\begin{array}{c}\text { ABTS }{ }^{+} \text {scavenging: } \mathrm{IC}_{50}=3.76 \pm 0.08 \mathrm{mM} \\
\text { DPPH scavenging: } \mathrm{IC}_{50}=3.26 \pm 0.04 \mathrm{mM} \\
\mathrm{Fe}^{2+} \text { chelation: } \mathrm{IC}_{50}=4.65 \pm 0.08 \mathrm{mM}\end{array}$ & [236] \\
\hline 285 & $\begin{array}{c}\text { K. alvarezii } \\
\text { (Rhodophyta, Florideophyceae, Gigartinales) }\end{array}$ & $\begin{array}{l}\text { ABTS }{ }^{+} \text {scavenging: } \mathrm{IC}_{50}=0.67 \pm 0.25 \mathrm{mM} \\
\text { DPPH scavenging: } \mathrm{IC}_{50}=0.61 \pm 0.06 \mathrm{mM}\end{array}$ & [234] \\
\hline 286 & $\begin{array}{c}\text { Gracilaria opuntia } \\
\text { (Rhodophyta, Florideophyceae, Gracilariales }\end{array}$ & $\begin{array}{l}\text { ABTS }{ }^{+} \text {scavenging: } \mathrm{IC}_{50}=0.50 \mathrm{mM} \\
\text { DPPH scavenging: } \mathrm{IC}_{50}=0.41 \mathrm{mM}\end{array}$ & [237] \\
\hline 287 & $\begin{array}{c}\text { C. trinodis } \\
\text { (Ochrophyta, Phaeophyceae, Fucales) } \\
\text { most probably as a contamination from Laurencia } \\
\text { sp. } \\
\text { (Rhodophyta, Florideophyceae, Ceramiales) }\end{array}$ & $\mathrm{ABTS}^{+}$scavenging: $26.01 \pm 0.01 \%$ & [135] \\
\hline 288 & $\begin{array}{c}\text { K. alvarezii } \\
\text { (Rhodophyta, Florideophyceae, Gigartinales) }\end{array}$ & $\begin{array}{l}\text { ABTS }{ }^{+} \text {scavenging: } \mathrm{IC}_{50}=1.30 \pm 0.48 \mathrm{mM} \\
\text { DPPH scavenging: } \mathrm{IC}_{50}=0.97 \pm 0.07 \mathrm{mM}\end{array}$ & [238] \\
\hline 289 & $\begin{array}{c}\text { K. alvarezii } \\
\text { (Rhodophyta, Florideophyceae, Gigartinales) }\end{array}$ & $\begin{array}{l}\text { ABTS }{ }^{+} \text {scavenging: } \mathrm{IC}_{50}=2.28 \mathrm{mM} \\
\text { DPPH scavenging: } \mathrm{IC}_{50}=2.02 \mathrm{mM}\end{array}$ & [235] \\
\hline 290 & $\begin{array}{c}\text { K. alvarezii } \\
\text { (Rhodophyta, Florideophyceae, Gigartinales) }\end{array}$ & $\begin{array}{l}\text { ABTS }+ \text { scavenging: } \mathrm{IC}_{50}=1.42 \mathrm{mM} \\
\text { DPPH scavenging: } \mathrm{IC}_{50}=2.50 \mathrm{mM}\end{array}$ & [235] \\
\hline 291 & $\begin{array}{c}\text { Spatoglossum variabile } \\
\text { (Ochrophyta, Phaeophyceae, Dictyotales) }\end{array}$ & $\mathrm{O}_{2}{ }^{-}$scavenging: $\mathrm{IC}_{50}=22.2 \mu \mathrm{M}$ & [239] \\
\hline 292 & $\begin{array}{c}\text { S. wightii } \\
\text { (Ochrophyta, Phaeophyceae, Fucales) }\end{array}$ & $\begin{array}{l}\text { ABTS }{ }^{+} \text {scavenging: } \mathrm{IC}_{50}=1.28 \pm 0.00 \mathrm{mM} \\
\text { DPPH scavenging: } \mathrm{IC}_{50}=1.05 \pm 0.03 \mathrm{mM}\end{array}$ & [240] \\
\hline 293 & $\begin{array}{c}\text { Hypnea musciformis } \\
\text { (Rhodophyta, Florideophyceae, Gigartinales) }\end{array}$ & $\begin{array}{c}\text { DPPH scavenging: } \mathrm{IC}_{50}=231.2 \pm 2.0 \mu \mathrm{M} \\
\mathrm{Fe}^{2+} \text { chelation: } \mathrm{IC}_{50}=667.9 \pm 0.8 \mu \mathrm{M} \\
\text { lipid peroxidation }(\mathrm{TBARS}) \\
1.34 \pm 0.01 \mathrm{MDAEQ} / \mathrm{kg} \text { at } 0.1 \mu \mathrm{g} / \mathrm{mL}\end{array}$ & [241] \\
\hline 294 & $\begin{array}{c}\text { S. wightii } \\
\text { (Ochrophyta, Phaeophyceae, Fucales) }\end{array}$ & $\begin{array}{c}\text { ABTS }{ }^{+} \text {scavenging: } \mathrm{IC}_{50}=1.81 \pm 0.03 \mathrm{mM} \\
\text { DPPH scavenging: } \mathrm{IC}_{50}=1.2 \pm 0.05 \mathrm{mM} \\
\mathrm{Fe}^{2+} \text { chelation: } \mathrm{IC}_{50}=2.28 \pm 0.03 \mathrm{mM}\end{array}$ & [236] \\
\hline 295 & $\begin{array}{c}\text { T. conoides } \\
\text { (Ochrophyta, Phaeophyceae, Fucales) }\end{array}$ & $\begin{array}{l}\text { ABTS }{ }^{+} \text {scavenging: } \mathrm{IC}_{50}=2.00 \mathrm{mM} \\
\text { DPPH scavenging: } \mathrm{IC}_{50}=1.71 \mathrm{mM}\end{array}$ & [242] \\
\hline 296 & $\begin{array}{c}\text { T. conoides } \\
\text { (Ochrophyta, Phaeophyceae, Fucales) }\end{array}$ & $\begin{array}{l}\text { ABTS }{ }^{+} \text {scavenging: } \mathrm{IC}_{50}=1.39 \mathrm{mM} \\
\text { DPPH scavenging: } \mathrm{IC}_{50}=1.29 \mathrm{mM}\end{array}$ & [242] \\
\hline 297 & $\begin{array}{c}\text { H. musciformis } \\
\text { (Rhodophyta, Florideophyceae, Gigartinales) }\end{array}$ & $\begin{array}{c}\text { DPPH scavenging: } \mathrm{IC}_{50}=25.0 \pm 0.5 \mu \mathrm{M} \\
\mathrm{Fe}^{2+} \text { chelation: } \mathrm{IC}_{50}=350.7 \pm 0.5 \mu \mathrm{M} \\
\text { lipid peroxidation (TBARS): } \\
0.88 \pm 0.01 \mathrm{MDAEQ} / \mathrm{kg} \text { at } 0.1 \mu \mathrm{g} / \mathrm{mL}\end{array}$ & [241] \\
\hline 298 & $\begin{array}{c}\text { H. musciformis } \\
\text { (Rhodophyta, Florideophyceae, Gigartinales) }\end{array}$ & $\begin{array}{c}\text { DPPH scavenging: } \mathrm{IC}_{50}=322.4 \pm 1.1 \mu \mathrm{M} \\
\mathrm{Fe}^{2+} \text { chelation: } \mathrm{IC}_{50}=5115.3 \pm 2.1 \mu \mathrm{M} \\
\text { lipid peroxidation (TBARS): } \\
0.76 \pm 0.01 \mathrm{MDAEQ} / \mathrm{kg} \text { at } 0.1 \mu \mathrm{g} / \mathrm{mL}\end{array}$ & [241] \\
\hline 299 & $\begin{array}{c}\text { S. wightii } \\
\text { (Ochrophyta, Phaeophyceae, Fucales) }\end{array}$ & $\begin{array}{c}\text { ABTS }{ }^{+} \text {scavenging: } \mathrm{IC}_{50}=0.81 \pm 0.04 \mathrm{mM} \\
\text { DPPH scavenging: } \mathrm{IC}_{50}=0.64 \pm 0.02 \mathrm{mM} \\
\mathrm{Fe}^{2+} \text { chelation: } \mathrm{IC}_{50}=1.42 \pm 0.02 \mathrm{mM}\end{array}$ & [236] \\
\hline 300 & $\begin{array}{c}\text { S. wightii } \\
\text { (Ochrophyta, Phaeophyceae, Fucales) }\end{array}$ & $\begin{array}{l}\text { ABTS }{ }^{+} \text {scavenging: } \mathrm{IC}_{50}=0.79 \pm 0.03 \mathrm{mM} \\
\text { DPPH scavenging: } \mathrm{IC}_{50}=0.67 \pm 0.03 \mathrm{mM}\end{array}$ & [240] \\
\hline
\end{tabular}


Table 7. Cont.

\begin{tabular}{ccccc}
\hline Compound & Isolation Source & Assay/Activity & Reference \\
\hline \multirow{2}{*}{301} & T. conoides & ABTS $^{+}$scavenging: $\mathrm{IC}_{50}=2.18 \mathrm{mM}$ & \\
& (Ochrophyta, Phaeophyceae, Fucales) & DPPH scavenging: $\mathrm{IC}_{50}=1.95 \mathrm{mM}$ & {$[242]$} \\
\hline
\end{tabular}

$\mathrm{ABTS}^{+}$: 2,2'-azino-bis (3-ethyl benzothiazoline-6-sulfonic acid) diammonium salt; AChE: acetylcholinesterase; BChE: butyrylcholinesterase; DPPH: 1,1-diphenyl-2-picrylhydrazyl free radical; $\mathrm{IC}_{50}$ : half maximal inhibitory concentration; $\mathrm{MDAEQ} / \mathrm{kg}$ : malondialdehyde equivalent compounds formed per kg sample; $\mathrm{ONOO}^{-}$: peroxynitrite; $\mathrm{O}_{2}{ }^{-}$: superoxide anion; TBARS: thiobarbituric acid reactive substances.<smiles>[R7]C(=CC(=O)O)CC</smiles>

272<smiles>CC(=O)/C=C/C=C/CCCC(=O)O</smiles>

273
$\mathrm{Me}\left(\mathrm{CH}_{2}\right)_{14} \mathrm{COOH}$

274
$\mathrm{Me}\left(\mathrm{CH}_{2}\right)_{7} \longrightarrow\left(\mathrm{CH}_{2}\right)_{7} \mathrm{COOH}$

275<smiles>C/C=C\C/C=C\C/C=C\C/C=C\C/C=C\CCCC(=O)O</smiles>

277

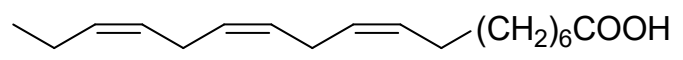

276<smiles>O=Cc1ccc(CO)o1</smiles><smiles>CC(O)/C=C/[C@]1(O)[C@H](C)CC(=O)CC1(C)C</smiles>

278

279<smiles>CCCC1CCCCC1CCCOC(=O)OC</smiles>

283<smiles>CCCC[C@H](CCOC(C)=O)COC</smiles>

281<smiles>C=C[C@H]1C[C@H]2CC=C3C[C@@H](O)CC[C@H]3[C@@]2(C)C[C@H]1OC</smiles>

282<smiles>CCCC(=CCCC(=O)OC[C@H](CC)CCCC(=O)OC[C@H](CCOC)C[C@@H]1CCOC(=O)C1)CC</smiles>

285<smiles>CC[C@H](C)[C@H](CCC(=O)OCC(O[C@H]1C2C=C[C@@H](C)C[C@H]2O[C@H](COC)[C@@H]1OC)[C@]1(C)CC(=O)OCC1OC(C)=O)OC(C)=O</smiles>

286<smiles>C#C/C=C\[C@H](Cl)C1C2CC(O2)C2O/C(=C(\Br)CC)C21</smiles>

287<smiles>CC[C@H](C)CC[C@@H]1C=CC2CCC(=O)O[C@@H]2C1</smiles>

284

Figure 23. Chemical structures of compounds 272-288. 


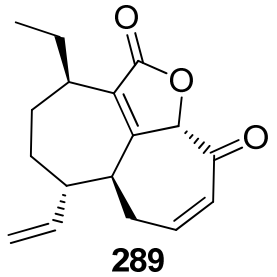<smiles>C=C[C@H]1CC=C(CC)C(C(=O)OC)[C@H]2CC(=O)C=CC[C@@H]21</smiles><smiles>O=C1/C(=C/c2ccc(Cl)cc2)Oc2ccccc21</smiles><smiles>CC[C@H]1C[C@H](CCCOC(=O)c2ccc(C)cc2)CC(=O)O1</smiles><smiles>CCCCC1C=C(C)C(CC(CCOC(=O)c2ccccc2)C(C)=O)OC1</smiles><smiles>CC[C@H](C)COC(=O)[C@@H]1CCC[C@](C)(CCCOC(=O)c2ccccc2)O1</smiles><smiles>CC[C@H](CCOC(=O)c1ccccc1)C[C@@H]1C(=O)OCC=C1C</smiles>

295<smiles>CC(=O)CC[C@H](C)[C@H]1OC[C@H](C[C@@H](C)CCOC(=O)c2ccc(O)cc2)C=C1C</smiles>

296<smiles>CCCCCC1C(CCOC(=O)c2ccc(O)cc2)COC1c1ccc(O)cc1</smiles><smiles>COC(CC(CCOC(=O)c1ccc(O)cc1)C(=O)O)C1=CCCC(OCCCC(C)C)O1</smiles><smiles>C=CC(C(C)C)C(OC(=O)[C@@H]1C=COC(CC(C)=O)[C@@H]1O)C(CC[18O])CC1Cc2ccccc2OC1=O</smiles><smiles>C=C[C@H]1/C=C/CC(=O)OCC(CC(=O)OC)/C=C/CC1c1ccccc1</smiles><smiles>CCC1=C[C@H](C)C(C)=CC(CCC(=O)O[C@@H]2CC[C@@H](C)OC2(C)C)C1</smiles>

300

301

Figure 24. Chemical structures of compounds 289-301.

Among these, the most active compounds, exerting significant DPPH radical scavenging capacity, were compounds 278 (5-hydroxymethyl-2-furfural, 5-HMF), 291 (Z-4' chloroaurone), and 297, with $\mathrm{IC}_{50}$ values at $27.1,22.2$, and $25.0 \mu \mathrm{M}$, respectively $[233,239,241]$. In particular, 5-HMF (278), isolated from L. undulata, exhibited significant antioxidant activities, as evaluated by its in vitro free radical species (including alkyl, DPPH, OH, and $\mathrm{O}_{2}{ }^{-}$ radicals) scavenging, intracellular ROS scavenging, membrane protein oxidation, MPO 
inhibition, as well as gene expression of the antioxidative enzymes GSH and SOD [233]. Overall, 5-HMF (278) displayed antioxidant activity, by scavenging overproducing free radicals and decreasing the activity of MPO or increasing the activity of GSH and SOD antioxidant enzymes in certain biological pathways.

Fang et al. (2010) isolated the non-polar compounds 272-274, 276, 277, and 279 from the red alga G. furcata and evaluated their antioxidant activities as inhibitors of AChE and $\mathrm{BChE}$ and as scavengers of DPPH radical and $\mathrm{ONOO}^{-}$[75]. All isolated compounds exhibited moderate $\mathrm{AChE}$ inhibitory activity with $\mathrm{IC}_{50}$ values ranging between 13.6 and $94.4 \mu \mathrm{M}$, whereas compounds $\mathbf{2 7 6}$ and 277 showed mild BChE inhibitory activity with $\mathrm{IC}_{50}$ values 57.1 and $21.7 \mu \mathrm{M}$, respectively. Only compound 272 showed substantial DPPH radical scavenging activity, while compounds 272 and 274 showed potent $\mathrm{ONOO}^{-}$ scavenging activity.

Compounds 280-286, 288-290, 292-296, and 298-301 exhibited moderate DPPH radical scavenging capacities, with $\mathrm{IC}_{50}$ values in the $\mathrm{mM}$ range, with activities comparable to either $\alpha$-tocopherol $\left(\mathrm{IC}_{50}=1.46 \mathrm{mM}\right)$, or BHT and BHA $\left(\mathrm{IC}_{50} \sim 1.30-1.54 \mathrm{mM}\right)$ [234-238,240-242]. Structure-activity relationship analysis revealed that the antioxidant activities of compounds 293,297 , and 298 were directly proportional to their steric freedom and hydrophobicity [241].

\section{Conclusions}

The marine environment harbors diverse biological species that can provide a vast repertoire of molecules with therapeutic properties. Forced to tolerate extreme environmental conditions, marine organisms produce structurally unique molecules as an adaptive strategy to survive in their biotopes. In particular, macroalgae contain a plethora of antioxidative compounds, such as bromophenols, phlorotannins, pigments, terpenoids, and polysaccharides, in order to protect themselves from free radicals, the production of which is favored in sublittoral zones with intense exposure to sunlight and high concentrations of oxygen.

Structural elements, such as the number of phenol rings, the number of free hydroxyl groups and conjugated systems, are in general accepted as enhancing the antioxidant activity observed. Among the metabolites presented in the current review, the most active belong to the classes of phenols and polyphenols, as well as meroterpenoids, with bromophenols and phlorotannins exerting the highest activities. In particular, the bromophenol rhodomelin A (18) isolated from the red alga $R$. confervoides, the phlorotannins fucodiphloroethol G (77), phlorofucofuroeckol-A (79), 974-B (83), and 2,7"-phloroglucinol6,6'-bieckol (84) purified from brown seaweeds especially of the genus Ecklonia, as well as the meroterpenoids 174, 175, and 178-183 isolated from brown algae of the genus Sargassum exerted noticeably high DPPH scavenging activity.

Nevertheless, the most studied antioxidant compounds are the natural pigments astaxanthin (116) and fucoxanthin (118), belonging to the class of carotenoids, ubiquitous in marine macroalgae. Their antioxidant action is based on their singlet oxygen quenching properties and their free radicals scavenging ability, which mainly depends on the number of conjugated double bonds and end groups. The antioxidant activity of fucoxanthin (118) has also been evaluated in vivo. Dietary intake of fucoxanthin significantly reduced lipid hydroperoxide levels of liver and abdominal white adipose tissue of obese/diabetes KK- $A^{y}$ mice [243]. Fucoxanthin supplementation also significantly reduced the blood glucose level and hepatic lipid contents of the mice. Promising results were also observed in experiments on rats fed a high fat diet supplemented with fucoxanthin that improved the antioxidant capacity, depleted by a high fat diet, by activating the Nrf2 pathway and its downstream target gene NQO1 [244]. Therefore, supplementation of the diet with fucoxanthin, especially of those who consume high fat in their diet, may benefit them by reducing the risk of oxidative stress.

Although emerging evidence points to a diversity of actions and effects, which are intricate and independent from any antioxidant chemical nature, there is an urgent need 
for deciphering the role of chemical structure on the antioxidant behavior of molecules. Moreover, constraints imposed by experimental protocols should always be taken into consideration when dealing with a lack of biological context in regard to results, so as to discriminate between the in vitro and in vivo scenarios. In this regard, the development of novel antioxidant activity detecting protocols prompts further investigations.

Author Contributions: Conceptualization, O.T., V.R. and E.I.; methodology, L.-A.T., V.R. and E.I.; investigation, L.-A.T. and M.A.T.; resources, V.R. and E.I.; writing-original draft preparation, L.-A.T. and M.A.T.; writing-review and editing, O.T., V.R. and E.I.; supervision, V.R. and E.I.; funding acquisition, V.R. and E.I. All authors have read and agreed to the published version of the manuscript.

Funding: This research has been co-financed by the European Union and Greek national funds through the Operational Program Competitiveness, Entrepreneurship and Innovation under the call SPECIAL ACTIONS “AQUACULTURE-INDUSTRIAL MATERIALS-OPEN INNOVATION IN CULTURE” (project code: T6ҮBП-00474, project acronym: ALGOSMETIC). The authors thank the Special Account for Research Grants of the National and Kapodistrian University of Athens for funding to cover the publication costs.

Institutional Review Board Statement: Not applicable.

Informed Consent Statement: Not applicable.

Data Availability Statement: Not applicable.

Acknowledgments: M.A.T. acknowledges support by the non-profit organization "Kleon Tsetis".

Conflicts of Interest: The authors declare no conflict of interest. The funders had no role in the design of the study; in the collection, analyses, or interpretation of data; in the writing of the manuscript, or in the decision to publish the results.

\section{References}

1. Nordberg, J.; Arnér, E.S. Reactive oxygen species, antioxidants, and the mammalian thioredoxin system. Free Radic. Biol. Med. 2001, 31, 1287-1312. [CrossRef]

2. Halliwell, B. Biochemistry of oxidative stress. Biochem. Soc. Trans. 2007, 35, 1147-1150. [CrossRef]

3. Valko, M.; Leibfritz, D.; Moncol, J.; Cronin, M.T.D.; Mazur, M.; Telser, J. Free radicals and antioxidants in normal physiological functions and human disease. Int. J. Biochem. Cell Biol. 2007, 39, 44-84. [CrossRef] [PubMed]

4. Rada, B.; Leto, T.L. Oxidative innate immune defenses by Nox/Duox family NADPH oxidases. Contrib. Microbiol. 2008, 15, 164-187. [CrossRef]

5. Devasagayam, T.P.A.; Tilak, J.C.; Boloor, K.K.; Sane, K.S.; Ghaskadbi, S.S.; Lele, R.D. Free radicals and antioxidants in human health: Current status and future prospects. J. Assoc. Phys. India 2004, 52, 794-804.

6. Gammone, M.; Riccioni, G.; D'Orazio, N. Marine carotenoids against oxidative stress: Effects on human health. Mar. Drugs 2015, 13, 6226-6246. [CrossRef] [PubMed]

7. Nimse, S.B.; Pal, D. Free radicals, natural antioxidants, and their reaction mechanisms. RSC Adv. 2015, 5, 27986-28006. [CrossRef]

8. Rahal, A.; Kumar, A.; Singh, V.; Yadav, B.; Tiwari, R.; Chakraborty, S.; Dhama, K. Oxidative stress, prooxidants, and antioxidants: The interplay. BioMed Res. Int. 2014, 2014, 761264. [CrossRef] [PubMed]

9. Valko, M.; Rhodes, C.J.; Moncol, J.; Izakovic, M.; Mazur, M. Free radicals, metals and antioxidants in oxidative stress-induced cancer. Chem. Biol. Interact. 2006, 160, 1-40. [CrossRef] [PubMed]

10. Palinski, W.; Rosenfeld, M.E.; Yla, H.S.; Gurtner, G.C.; Socher, S.S.; Butler, S.W.; Carew, T.E.; Parthasarathy, S.; Steinberg, D.; Witztum, J.L. Low density lipoprotein undergoes oxidative modification in vivo. Proc. Natl. Acad. Sci. USA 1989, 86, 1372-1376. [CrossRef]

11. Golbidi, S.; Ebadi, S.A.; Laher, I. Antioxidants in the treatment of diabetes. Curr. Diabetes Rev. 2011, 7, 106-125. [CrossRef]

12. Bodamyali, T.; Kanczler, J.M.; Millar, T.M.; Stevens, C.R.; Blake, D.R. Free radicals in rheumatoid arthritis: Mediators and modulators. Oxid. Stress Dis. 2004, 10, 591-610.

13. Cuzzocrea, S.; Riley, D.P.; Caputi, A.P.; Salvemini, D. Antioxidant therapy: A new pharmacological approach in shock, inflammation, and ischemia/reperfusion injury. Pharmacol. Rev. 2001, 53, 135-159. [PubMed]

14. Gandhi, S.; Abramov, A.Y. Mechanism of oxidative stress in neurodegeneration. Oxid. Med. Cell. Longev. 2012, $2012,428010$. [CrossRef]

15. Traysman, R.J.; Kirsch, J.R.; Koehler, R.C. Oxygen radical mechanisms of brain injury following ischemia and reperfusion. J. Appl. Physiol. 1991, 71, 1185-1195. [CrossRef]

16. Acharya, A.; Das, I.; Chandhok, D.; Saha, T. Redox regulation in cancer: A double-edged sword with therapeutic potential. Oxid. Med. Cell. Longev. 2010, 3, 23-34. [CrossRef] 
17. Pisoschi, A.M.; Pop, A. The role of antioxidants in the chemistry of oxidative stress: A review. Eur. J. Med. Chem. 2015, 97, 55-74. [CrossRef] [PubMed]

18. Sulthana, S.M.; Kumar, S.N.; Sridhar, M.G.; Bhat, B.V.; Rao, K.R. Levels of non enzymatic antioxidants in Down syndrome. Indian J. Pediatr. 2012, 79, 1473-1476. [CrossRef]

19. Chen, H.; Yu, M.; Li, M.; Zhao, R.; Zhu, Q.; Zhou, W.; Lu, M.; Lu, Y.; Zheng, T.; Jiang, J.; et al. Polymorphic variations in manganese superoxide dismutase (MnSOD), glutathione peroxidase-1 (GPX1), and catalase (CAT) contribute to elevated plasma triglyceride levels in Chinese patients with type 2 diabetes or diabetic cardiovascular disease. Mol. Cell. Biochem. 2012, 363, 85-91. [CrossRef]

20. Young, I.; Woodside, J. Antioxidants in health and disease. J. Clin. Pathol. 2001, 54, 176-186. [CrossRef] [PubMed]

21. Poljsak, B.; Suput, D.; Milisav, I. Achieving the balance between ROS and antioxidants: When to use the synthetic antioxidants. Oxid. Med. Cell. Longev. 2013, 2013, 956792. [CrossRef] [PubMed]

22. Kim, S.K.; Mendis, E. Bioactive compounds from marine processing by products-a review. Food Res. Int. 2006, 39, 383-393. [CrossRef]

23. Ahmad, B.; Shah, M.; Choi, S. Oceans as a source of immunotherapy. Mar. Drugs 2019, 17, 282. [CrossRef] [PubMed]

24. Carroll, A.R.; Copp, B.R.; Davis, R.A.; Keyzers, R.A.; Prinsep, M.R. Marine natural products. Nat. Prod. Rep. 2021, $38,362-413$. [CrossRef]

25. Choudhary, A.; Naughton, L.M.; Montánchez, I.; Dobson, A.D.W.; Rai, D.K. Current status and future prospects of marine natural products (MNPs) as antimicrobials. Mar. Drugs 2017, 15, 272. [CrossRef]

26. Khalifa, S.A.M.; Elias, N.; Farag, M.A.; Chen, L.; Saeed, A.; Hegazy, M.-E.F.; Moustafa, M.S.; Abd El-Wahed, A.; Al-Mousawi, S.M.; Musharraf, S.G.; et al. Marine natural products: A source of novel anticancer drugs. Mar. Drugs 2019, 17, 491. [CrossRef]

27. Li, T.; Ding, T.; Li, J. Medicinal purposes: Bioactive metabolites from marine-derived organisms. Mini-Rev. Med. Chem. 2019, 19, 138-164. [CrossRef]

28. MarinLit. A Database of the Marine Natural Products Literature. Available online: http://pubs.rsc:marinlit/ (accessed on 31 March 2021).

29. Barzkar, N.; Jahromi, S.T.; Poorsaheli, H.B.; Vianello, F. Metabolites from marine microorganisms, micro, and macroalgae: Immense scope for pharmacology. Mar. Drugs 2019, 17, 464. [CrossRef]

30. Matsukawa, R.; Dubinsky, Z.; Kishimoto, E.; Masaki, K.; Masuda, Y.; Takeuchi, T. A comparison of screening methods for antioxidant activity in seaweeds. J. Appl. Phycol. 1997, 9, 29-35. [CrossRef]

31. Athiperumalsami, T.; Rajeswari, V.D.; Poorna, S.H.; Kumar, V.; Jesudass, L.L. Antioxidant activity of seagrasses and seaweeds. Bot. Mar. 2010, 53, 251-257. [CrossRef]

32. Kelman, D.; Posner, E.K.; McDermid, K.J.; Tabandera, N.K.; Wright, P.R.; Wright, A.D. Antioxidant activity of Hawaiian marine algae. Mar. Drugs 2012, 10, 403-416. [CrossRef]

33. Zubia, M.; Robledo, D.; Freile-Pelegrin, Y. Antioxidant activities in tropical marine macroalgae from the Yucatan Peninsula. J. Appl. Phycol. 2007, 19, 449-458. [CrossRef]

34. Sansone, C.; Brunet, C. Marine algal antioxidants. Antioxidants 2020, 9, 206. [CrossRef]

35. Fernando, I.P.S.; Kim, M.; Son, K.-T.; Jeong, Y.; Jeon, Y.-J. Antioxidant activity of marine algal polyphenolic compounds: A mechanistic approach. J. Med. Food 2016, 19, 1-14. [CrossRef]

36. Jacobsen, C.; Sørensen, A.-D.M.; Holdt, S.L.; Akoh, C.C.; Hermund, D.B. Source, extraction, characterization, and applications of novel antioxidants from seaweed. Annu. Rev. Food Sci. Technol. 2019, 10, 26.1-26.28. [CrossRef]

37. Jiao, G.-L.; Yu, G.L.; Zhao, X.-L.; Zhang, J.-Z.; Ewart, H.S. Natural polymers with antioxidant properties: Poly-/oligosaccharides of marine origin. In Antioxidant Polymers: Synthesis, Properties, and Applications, 1st ed.; Cirillo, G., Lemma, F., Eds.; Wiley, Scrivener Publishing LLC: Beverly, MA, USA, 2012; pp. 179-202.

38. Schlesier, K.; Harwat, M.; Böhm, V.; Bitsch, R. Assessment of antioxidant activity by using different in vitro methods. Free Radic. Res. 2002, 36, 177-187. [CrossRef] [PubMed]

39. Huang, D.; Ou, B.; Prior, R.L. The chemistry behind antioxidant capacity assays. J. Agric. Food Chem. 2005, 53, 1841-1856. [CrossRef] [PubMed]

40. Cao, G.; Prior, R.L. Measurement of oxygen radical absorbance capacity in biological samples. Meth. Enzymol. 1999, 299, 50-62. [CrossRef]

41. Wayner, D.D.M.; Burton, G.W.; Ingold, K.U.; Locke, S. Quantitative measurement of the total, peroxyl radical-trapping antioxidant capability of human blood plasma by controlled peroxidation. The important contribution made by plasma proteins. FEBS Lett. 1985, 187, 33-37. [CrossRef]

42. Singleton, V.L.; Orthofer, R.; Lamuela-Raventos, R.M. Analysis of total phenols and other oxidation substrates and antioxidants by means of folin-ciocalteu reagent. Meth. Enzymol. 1999, 299, 152-178. [CrossRef]

43. Miller, N.J.; Rice-Evans, C.A.; Davies, M.J.; Gopinathan, V.; Milner, A. A novel method for measuring antioxidant capacity and its application to monitoring the antioxidant status in premature neonates. Clin. Sci. 1993, 84, 407-412. [CrossRef]

44. Benzie, I.F.; Strain, J.J. The ferric reducing ability of plasma (FRAP) as a measure of "antioxidant power": The FRAP assay. Anal. Biochem. 1996, 239, 70-76. [CrossRef] 
45. Benzie, I.F.; Strain, J.J. Ferric reducing/antioxidant power assay: Direct measure of total antioxidant activity of biological fluids and modified version for simultaneous measurement of total antioxidant power and ascorbic acid concentration. Methods Enzymol. 1999, 299, 15-27. [CrossRef]

46. Blois, M.S. Antioxidant determinations by the use of a stable free radical. Nature 1958, 181, 1199-1200. [CrossRef]

47. Brand-Williams, W.; Cuvelier, M.E.; Berset, C. Use of a free radical method to evaluate antioxidant activity. LWT-Food Sci. Technol. Lebensm. Wiss. Technol. 1995, 28, 25-30. [CrossRef]

48. Zhang, Q.; Li, N.; Zhou, G.; Lu, X.; Xu, Z.; Li, Z. In vivo antioxidant activity of polysaccharide fraction from Porphyra haitanesis (Rhodophyta) in aging mice. Pharmacol. Res. 2003, 48, 151-155. [CrossRef]

49. Zhang, Q.; Li, N.; Liu, X.; Zhao, Z.; Li, Z.; Xu, Z. The structure of a sulfated galactan from Porphyra haitanensis and its in vivo antioxidant activity. Carbohydr. Res. 2004, 339, 105-111. [CrossRef]

50. Dontha, S. A review on antioxidant methods. Asian J. Pharm. Clin. Res. 2016, 9, 14-32. [CrossRef]

51. Re, R.; Pellegrini, N.; Proteggente, A.; Pannala, A.; Yang, M.; Rice-Evans, C.A. Antioxidant activity applying an improved ABTS radical cation decolorization assay. Free Radic. Biol. Med. 1999, 26, 1231-1237. [CrossRef]

52. Miller, H.E. A simplified method for the evaluation of antioxidants. J. Am. Oil Chem. Soc. 1971, 48, 91. [CrossRef]

53. Bors, W.; Michel, C.; Saran, M. Inhibition of bleaching of the carotenoid crocin, a rapid test for quantifying antioxidant activity. Biochim. Biophys. Acta 1984, 796, 312-319. [CrossRef]

54. Winston, G.W.; Regoli, F.; Dugas, A.J., Jr.; Fong, J.H.; Blanchard, K.A. A rapid gas chromatographic assay for determining oxyradical scavenging capacity of antioxidants and biological fluids. Free Radic. Biol. Med. 1998, 24, 480-493. [CrossRef]

55. Ou, B.; Hampsch-Woodill, M.; Flanagan, J.; Deemer, E.K.; Prior, R.L.; Huang, D. Novel fluorometric assay for hydroxyl radical prevention capacity using fluorescein as the probe. J. Agric. Food Chem. 2002, 50, 2772-2777. [CrossRef]

56. Kunchandy, E.; Rao, M.N. Oxygen radical scavenging activity of curcumin. Int. J. Pharm. 1990, 58, 237-240. [CrossRef]

57. Burton, G.W.; Ingold, K.U. Autoxidation of biological molecules. 1. The autoxidation of vitamin E and related chainbreaking antioxidants in vitro. J. Am. Chem. Soc. 1981, 103, 6472-6477. [CrossRef]

58. Okawa, H.; Ohishi, N.; Yagi, K. Assay for lipid peroxides in animal tissues by thiobarbituric acid reaction. Anal. Biochem. 1979, 95, 351-358. [CrossRef]

59. Popov, I.; Lewin, G. Antioxidative homeostasis: Characterization by means of chemiluminescent technique. Methods Enzymol. 1999, 300, 437-456.

60. Apak, R.; Güçlü, K.; Özyürek, M.; Karademir, S.E. A novel total antioxidant capacity index for dietary polyphenols, vitamins c and e, using their cupric ion reducing capability in the presence of neocuproine: CUPRAC method. J. Agric. Food Chem. 2004, 52, 7970-7981. [CrossRef]

61. Kikuzaki, H.; Usuguchi, J.; Nakatani, N. Constituents of Zingiberaceae. I. Diarylheptanoids from the rhizomes of ginger (Zingiber officinale roscoe). Chem. Pharm. Bull. 1991, 39, 120-122. [CrossRef]

62. Marcocci, L.; Maguire, J.J.; Droy-Lefaix, M.T.; Packer, L. The nitric oxide scavenging property of Ginkgo biloba extract EGB 761. Biochem. Biophys. Res. Commun. 1994, 201, 748-755. [CrossRef]

63. Verde, V.; Fogliano, V.; Ritieni, A.; Maiani, G.; Morisco, F.; Caporaso, N. Use of N,N-dimethyl-p-phenylenediamine to evaluate the oxidative status of human plasma. Free Radic. Res. 2002, 36, 869-873. [CrossRef] [PubMed]

64. Maitra, I.; Marcocci, L.; Droy-Lefaix, M.T.; Packer, L. Peroxyl radical scavenging activity of Ginkgo biloba extract EGb 761. Biochem. Pharmacol. 1995, 49, 1649-1655. [CrossRef]

65. Oyaizu, M. Studies on products of browning reactions-antioxidative activities of products of browning reaction prepared from glucosamine. Jpn. J. Nutr. 1986, 44, 307-315. [CrossRef]

66. Korycka-Dahl, M.; Richardson, M. Photogeneration of superoxide anion in serum of bovine milk and in model systems containing riboflavin and aminoacids. J. Dairy Sci. 1978, 61, 400-407. [CrossRef]

67. Yagi, K. A simple fluorometric assay for lipoperoxide in blood plasma. Biochem. Med. 1976, 15, 212-216. [CrossRef]

68. Lee, H.S.; Coates, G.A. Measurement of total vitamin C activity in citrus products by HPLC. J. Liq. Chromatogr. Relat. Technol. 1999, 22, 2367-2387. [CrossRef]

69. Gonzalez, E.; Vaillant, F.; Rojas, G.; Pérez, A. Novel semiautomated method for assessing in vitro cellular antioxidant activity using the light scattering properties of human erythrocytes. J. Agric. Food Chem. 2010, 58, 1455-1461. [CrossRef]

70. Carter, P. Spectrophotometric determination of serum iron at the submicrogram level with a new reagent (Ferrozine). Anal. Biochem. 1971, 40, 450-458. [CrossRef]

71. Prieto, P.; Pineda, M.; Aguilar, M. Spectrophotometric quantitation of antioxidant capacity through the formation of a phosphomolybdenum complex: Specific application to the determination of vitamin E. Anal. Biochem. 1999, 269, 337-341. [CrossRef]

72. Noro, T.; Oda, Y.; Miyase, T.; Ueno, A.; Fukushima, S. Inhibitors of xanthine oxidase from the flowers and buds of Daphne genkwa. Chem. Pharm. Bull. 1983, 31, 3984-3987. [CrossRef]

73. Liu, M.; Hansen, P.E.; Lin, X. Bromophenols in marine algae and their bioactivities. Mar. Drugs 2011, 9, 1273-1292. [CrossRef]

74. Duan, X.J.; Li, X.M.; Wang, B.G. Highly brominated mono- and bis-phenols from the marine red alga Symphyocladia latiuscula with radical-scavenging activity. J. Nat. Prod. 2007, 70, 1210-1213. [CrossRef]

75. Fang, Z.; Jeong, S.Y.; Jung, H.A.; Choi, J.S.; Min, B.S.; Woo, M.H. Anticholinesterase and antioxidant constituents from Gloiopeltis furcata. Chem. Pharm. Bull. 2010, 58, 1239. [CrossRef] 
76. Li, K.; Li, X.M.; Gloer, J.B.; Wang, B.G. Isolation, characterization, and antioxidant activity of bromophenols of the marine red alga Rhodomela confervoides. J. Agric. Food Chem. 2011, 59, 9916-9921. [CrossRef]

77. Lee, J.H.; Lee, T.K.; Kang, R.S.; Shin, H.J.; Lee, H.S. The in vitro antioxidant activities of the bromophenols from the red alga Tichocarpus crinitus and phenolic derivatives. J. Korean Magn. Reson. Soc. 2007, 11, 56-63.

78. Park, H.J.; Kim, H.R.; Choi, J.S. Antioxidant effect of 2,3,6-tribromo-4,5-dihydroxybenzyl methyl ether (TDB) from the red alga, Symphyocladia latiuscula. J. Fish. Sci. Technol. 2009, 12, 86-89. [CrossRef]

79. Choi, J.S.; Park, H.J.; Jung, H.A.; Chung, H.Y.; Jung, J.H.; Choi, W.C. A cyclohexanonyl bromophenol from the red alga Symphyocladia latiuscula. J. Nat. Prod. 2000, 63, 1705-1706. [CrossRef]

80. Rezai, M.; Bayrak, Ç.; Taslimi, P.; Gülçin, I.; Menzek, A. The first synthesis and antioxidant and anticholinergic activities of 1-(4,5-dihydroxybenzyl)pyrrolidin-2-one derivative bromophenols including natural products. Turk. J. Chem. 2018, 42, 808-825.

81. Li, K.; Li, X.M.; Gloer, J.B.; Wang, B.G. New nitrogen-containing bromophenols from the marine red alga Rhodomela confervoides and their radical scavenging activity. Food Chem. 2012, 135, 868-872. [CrossRef]

82. Xu, X.L.; Yin, L.Y.; Gao, J.H.; Chen, J.H.; Li, J.X.; Song, F.H. Two new bromophenols with radical scavenging activity from marine red alga Symphyocladia latiuscula. Mar. Drugs 2013, 11, 842-847. [CrossRef] [PubMed]

83. Li, K.; Wang, Y.F.; Li, X.M.; Wang, W.J.; Ai, X.Z.; Li, X.; Yang, S.Q.; Gloer, J.B.; Wang, B.G.; Xu, T. Isolation, synthesis, and radical-scavenging activity of rhodomelin A, a ureidobromophenol from the marine red alga Rhodomela confervoides. Org. Lett. 2018, 20, 417-420. [CrossRef]

84. Li, K.; Li, X.M.; Ji, N.Y.; Wang, B.G. Bromophenols from the marine red alga Polysiphonia urceolata with DPPH radical scavenging activity. J. Nat. Prod. 2008, 71, 28-30. [CrossRef]

85. Ryu, Y.S.; Fernando, P.D.S.M.; Kang, K.A.; Piao, M.J.; Zhen, A.X.; Kang, H.K.; Koh, Y.S.; Hyun, J.W. Marine compound 3-bromo4,5-dihydroxybenzaldehyde protects skin cells against oxidative damage via the Nrf2/HO-1 pathway. Mar. Drugs 2019, 17, 234. [CrossRef]

86. Olsen, E.K.; Hansen, E.; Isaksson, J.; Andersen, J.H. Cellular antioxidant effect of four bromophenols from the red algae, Vertebrata lanosa. Mar. Drugs 2013, 11, 2769-2784. [CrossRef]

87. Cha, J.W.; Piao, M.J.; Kim, K.C.; Zheng, J.; Yao, C.W.; Hyun, C.L.; Kang, H.K.; Yoo, E.S.; Young, S.K.; Lee, N.H.; et al. Protective effect of 3,4-dihydroxybenzoic acid isolated from Cladophora wrightiana Harvey against ultraviolet B radiation-induced cell damage in human HaCaT keratinocytes. Appl. Biochem. Biotechnol. 2014, 172, 2582-2592. [CrossRef]

88. Li, K.; Li, X.M.; Ji, N.Y.; Wang, B.G. Natural bromophenols from the marine red alga Polysiphonia urceolata (Rhodomelaceae): Structural elucidation and DPPH radical-scavenging activity. Bioorg. Med. Chem. 2007, 15, 6627-6631. [CrossRef]

89. Islam, M.R.; Mikami, D.; Kurihara, H. Two new algal bromophenols from Odonthalia corymbifera. Tetrahedron Lett. 2017, 58, 4119-4121. [CrossRef]

90. Choi, Y.K.; Ye, B.R.; Kim, E.A.; Kim, J.; Kim, M.S.; Lee, W.W.; Ahn, G.N.; Kang, N.; Jung, W.K.; Heo, S.J. Bis (3-bromo-4,5dihydroxybenzyl) ether, a novel bromophenol from the marine red alga Polysiphonia morrowii that suppresses LPS-induced inflammatory response by inhibiting ROS-mediated ERK signaling pathway in RAW 264.7 macrophages. Biomed. Pharmacother. 2018, 103, 1170-1177. [CrossRef]

91. Takamatsu, S.; Hodges, T.W.; Rajbhandari, I.; Gerwick, W.H.; Hamann, M.T.; Nagle, D.G. Marine natural products as novel antioxidant prototypes. J. Nat. Prod. 2003, 66, 605-608. [CrossRef]

92. Li, K.; Li, X.M.; Ji, N.Y.; Gloer, J.B.; Wang, B.G. Urceolatin, a structurally unique bromophenol from Polysiphonia urceolata. Org. Lett. 2008, 7, 1429-1432. [CrossRef]

93. Kang, K.A.; Lee, K.H.; Chae, S.; Zhang, R.; Jung, M.S.; Ham, Y.M.; Baik, J.S.; Lee, N.H.; Hyun, J.W. Cytoprotective effect of phloroglucinol on oxidative stress induced cell damage via catalase activation. J. Cell. Biochem. 2006, 97, 609-620. [CrossRef]

94. Karthik, P.; Manigandan, V.; Sheeba, R.; Saravanan, R.; Rajesh, P.R. Structural characterization and comparative biomedical properties of phloroglucinol from Indian brown seaweeds. J. Appl. Phycol. 2016, 28, 3561-3573. [CrossRef]

95. Li, Y.; Qian, Z.J.; Ryu, B.; Lee, S.H.; Kim, M.M.; Kim, S.K. Chemical components and its antioxidant properties in vitro: An edible marine brown alga, Ecklonia cava. Bioorg. Med. Chem. 2009, 17, 1963-1973. [CrossRef]

96. Park, C.; Cha, H.-J.; Hong, S.H.; Kim, G.-Y.; Kim, S.; Kim, H.-S.; Kim, B.W.; Jeon, Y.-J.; Choi, Y.H. Protective effect of phloroglucinol on oxidative stress-induced DNA damage and apoptosis through activation of the Nrf2/HO-1 signaling pathway in HaCaT human keratinocytes. Mar. Drugs 2019, 17, 225. [CrossRef] [PubMed]

97. Zou, Y.; Qian, Z.J.; Li, Y.; Kim, M.M.; Lee, S.H.; Kim, S.K. Antioxidant Effects of phlorotannins isolated from Ishige okamurae in free radical mediated oxidative systems. J. Agric. Food Chem. 2008, 56, 7001-7009. [CrossRef] [PubMed]

98. Andriani, Y.; Syamsumir, D.F.; Yee, T.C.; Harisson, F.S.; Herng, G.M.; Abdullah, S.A.; Orosco, C.A.; Ali, A.M.; Latip, J.; Kikuzaki, H.; et al. Biological activities of isolated compounds from three edible Malaysian red seaweeds, Gracilaria changii, G. manilaensis and Gracilaria sp. Nat. Prod. Commun. 2016, 11, 1117-1120. [CrossRef]

99. Kim, C.; Lee, I.K.; Cho, G.Y.; Oh, K.H.; Lim, Y.W.; Yun, B.S. Sargassumol, a novel antioxidant from the brown alga Sargassum micracanthum. J. Antibiot. 2012, 65, 87-89. [CrossRef]

100. Kim, K.C.; Lee, I.K.; Kang, K.A.; Piao, M.J.; Ryu, M.J.; Kim, J.M.; Lee, N.H.; Hyun, J.W. Triphlorethol-A from Ecklonia cava up-regulates the oxidant sensitive 8-oxoguanine DNA glycosylase 1. Mar. Drugs 2014, 12, 5357-5371. [CrossRef] 
101. Kang, M.C.; Kim, K.N.; Lakmal, H.H.C.; Kim, E.A.; Wijesinghe, W.A.J.P.; Yang, X.; Heo, S.J.; Jeon, Y.J. Octaphlorethol A isolated from Ishige foliacea prevents and protects against high glucose-induced oxidative damage in vitro and in vivo. Environ. Toxicol. Pharmacol. 2014, 38, 607-615. [CrossRef]

102. Lee, S.H.; Kang, S.M.; Ko, S.C.; Kang, M.C.; Jeon, Y.J. Octaphlorethol A, a novel phenolic compound isolated from Ishige foliacea, protects against streptozotocin-induced pancreatic cell damage by reducing oxidative stress and apoptosis. Food Chem. Toxicol. 2013, 643-649. [CrossRef]

103. Jun, Y.J.; Lee, M.; Shin, T.; Yoon, N.; Kim, J.H.; Kim, H.R. Eckol enhances heme oxygenase-1 expression through activation of Nrf2/JNK pathway in HepG2 cells. Molecules 2014, 19, 15638-15652. [CrossRef] [PubMed]

104. Kang, H.S.; Chung, H.Y.; Jung, J.H.; Son, B.W.; Choi, J.S. A new phlorotannin from the brown alga Ecklonia stolonifera. Chem. Pharm. Bull. 2003, 51, 1012-1014. [CrossRef]

105. Kang, H.S.; Chung, H.Y.; Kim, J.Y.; Son, B.W.; Jung, H.A.; Choi, J.S. Inhibitory phlorotannins from the edible brown alga Ecklonia stolonifera on total reactive oxygen species (ROS) generation. Arch. Pharm. Res. 2004, 27, 194-198. [CrossRef] [PubMed]

106. Kang, K.A.; Lee, K.H.; Chae, S.; Zhang, R.; Jung, M.S.; Lee, Y.; Kim, S.Y.; Kim, H.S.; Joo, H.G.; Park, J.W.; et al. Eckol isolated from Ecklonia cava attenuates oxidative stress induced cell damage in lung fibroblast cells. FEBS Lett. 2005, 579, 6295-6304. [CrossRef]

107. Lee, J.W.; Seok, J.K.; Boo, Y.C. Ecklonia cava extract and dieckol attenuate cellular lipid peroxidation in keratinocytes exposed to PM10. J. Evid. Based Complementary Altern. Med. 2018, 2018, 8248323. [CrossRef] [PubMed]

108. Shibata, T.; Ishimaru, K.; Kawaguchi, S.; Yoshikawa, H.; Hama, Y. Antioxidant activities of phlorotannins isolated from Japanese Laminariaceae. J. Appl. Phycol. 2008, 20, 705-711. [CrossRef]

109. Kim, A.R.; Shin, T.S.; Lee, M.S.; Park, J.Y.; Park, K.E.; Yoon, N.Y.; Kim, J.S.; Choi, J.S.; Jang, B.C.; Byun, D.S.; et al. Isolation and identification of phlorotannins from Ecklonia stolonifera with antioxidant and anti-inflammatory properties. J. Agric. Food Chem. 2009, 57, 3483-3489. [CrossRef]

110. Heo, S.J.; Cha, S.H.; Kim, K.N.; Lee, S.H.; Ahn, G.; Kang, D.H.; Oh, C.; Choi, Y.U.; Affan, A.; Kim, D.; et al. Neuroprotective effect of phlorotannin isolated from Ishige okamurae against $\mathrm{H}_{2} \mathrm{O}_{2}$-induced oxidative stress in murine hippocampal neuronal cells, $\mathrm{HT22}$. Appl. Biochem. Biotechnol. 2012, 166, 1520-1532. [CrossRef]

111. Zhen, A.X.; Piao, M.J.; Hyun, Y.J.; Kang, K.A.; Fernando, P.D.S.M.; Cho, S.J.; Ahn, M.J.; Hyun, J.W. Diphlorethohydroxycarmalol attenuates fine particulate matter-induced subcellular skin dysfunction. Mar. Drugs 2019, 17, 95. [CrossRef]

112. Cha, S.H.; Heo, S.J.; Jeon, Y.J.; Park, S.M. Dieckol, an edible seaweed polyphenol, retards rotenone-induced neurotoxicity and $\alpha$-synuclein aggregation in human dopaminergic neuronal cells. RSC Adv. 2016, 6, 110040-110046. [CrossRef]

113. Yoon, J.S.; Yadunandam, A.K.; Kim, S.J.; Woo, H.C.; Kim, H.R.; Kim, G.D. Dieckol, isolated from Ecklonia stolonifera, induces apoptosis in human hepatocellular carcinoma Hep3B cells. J. Nat. Med. 2013, 67, 519-527. [CrossRef] [PubMed]

114. Cérantola, S.; Breton, F.; Gall, A.E.; Deslandes, E. Co-occurrence and antioxidant activities of fucol and fucophlorethol classes of polymeric phenols in Fucus spiralis. Bot. Mar. 2006, 49, 347-351. [CrossRef]

115. Ham, Y.M.; Baik, J.S.; Hyun, J.W.; Lee, N.H. Isolation of a new phlorotannin, fucodiphlorethol G, from a brown alga Ecklonia cava. Bull. Korean Chem. Soc. 2007, 28, 1595-1597.

116. Kim, K.C.; Piao, M.J.; Zheng, J.; Yao, C.W.; Cha, J.W.; Kumara, M.H.S.R.; Han, X.; Kang, H.K.; Lee, N.H.; Hyun, J.W. Fucodiphlorethol $\mathrm{G}$ purified from Ecklonia cava suppresses ultraviolet B radiation-induced oxidative stress and cellular damage. Biomol. Ther. 2014, 22, 301-307. [CrossRef]

117. Parys, S.; Kehraus, S.; Krick, A.; Glombitza, K.; Carmeli, S.; Klimo, K.; Gerhaeuser, C.; Koenig, G.M. In vitro chemopreventive potential of fucophlorethols from the brown alga Fucus vesiculosus L. by anti-oxidant activity and inhibition of selected cytochrome P450 enzymes. Phytochemistry 2010, 71, 221-229. [CrossRef]

118. Lee, J.H.; Ko, J.Y.; Oh, J.Y.; Kim, E.A.; Kim, C.Y.; Jeon, Y.J. Evaluation of phlorofucofuroeckol-A isolated from Ecklonia cava (Phaeophyta) on anti-lipid peroxidation in vitro and in vivo. Algae 2015, 30,313-323. [CrossRef]

119. Kwon, T.H.; Suh, H.J.; Lee, I.K.; Yun, B.S.; Kim, T.W.; Hwang, D.I.; Kim, Y.J.; Kim, M.J.; Kwon, O.O.; Kim, C.G.; et al. Determination of singlet oxygen quenching and antioxidant activity of Bieckols isolated from the brown alga Eisenia bicyclis. Eur. Food Res. Technol. 2013, 237, 501-508. [CrossRef]

120. Lee, B.H.; Choi, B.W.; Lee, S.Y. Isolation of 6,6'-bieckol from Grateloupia elliptica and its antioxidative and anti-cholinesterase activity. Ocean Polar Res. 2017, 39, 45-49. [CrossRef]

121. Park, M.H.; Heo, S.J.; Park, P.J.; Moon, S.H.; Sung, S.H.; Jeon, B.T.; Lee, S.H. 6,6'-Bieckol isolated from Ecklonia cava protects oxidative stress through inhibiting expression of ROS and proinflammatory enzymes in high-glucose-induced human umbilical vein endothelial cells. Appl. Biochem. Biotechnol. 2014, 174, 632-643. [CrossRef] [PubMed]

122. Choi, J.S.; Haulader, S.; Karki, S.; Jung, H.J.; Kim, H.R.; Jung, H.A. Acetyl- and butyryl-cholinesterase inhibitory activities of the edible brown alga Eisenia bicyclis. Arch. Pharm. Res. 2015, 38, 1477-1487. [CrossRef] [PubMed]

123. Kang, S.M.; Heo, S.J.; Kim, K.N.; Lee, S.H.; Jeon, Y.J. Isolation and identification of new compound, 2,7"-phloroglucinol-6,6'-bieckol from brown algae, Ecklonia cava and its antioxidant effect. J. Funct. Foods 2012, 4, 158-166. [CrossRef]

124. Zeng, L.M.; Wang, C.J.; Yu, S.J.; Li, D.; Owen, N.L.; Lu, Y.; Lu, N.; Zheng, Q.T. Flavonoids from the red alga Acanthophora spicifera. Chin. J. Chem. 2001, 19, 1097-1100. [CrossRef]

125. Ragan, M.A.; Glombitza, K.W. Phlorotannins, brown algal polyphenols. In Progress in Phycological Research; Round, F.E., Chapman, D.J., Eds.; Biopress Ltd.: Bristol, UK, 1986; pp. 129-241.

126. Singh, I.P.; Bharate, S.B. Phloroglucinol compounds of natural origin. Nat. Prod. Rep. 2006, 23, 558-591. [CrossRef] 
127. Gross, H.; König, G.M. Terpenoids from marine organisms: Unique structures and their pharmacological potential. Phytochem. Rev. 2006, 5, 115-141. [CrossRef]

128. Shapumba, C.W.; Knott, M.; Kapewangolo, P. Antioxidant activity of a halogenated monoterpene isolated from a Namibian marine algal Plocamium species. J. Food Sci. Technol. 2017, 54, 3370-3373. [CrossRef]

129. Chakraborty, K.; Paulraj, R. Sesquiterpenoids with free-radical-scavenging properties from marine macroalga Ulva fasciata Delile. Food Chem. 2010, 122, 31-41. [CrossRef]

130. Guajardo, E.; Correa, J.A.; Contreras-Porcia, L. Role of abscisic acid (ABA) in activating antioxidant tolerance responses to desiccation stress in intertidal seaweed species. Planta 2016, 243, 767-781. [CrossRef]

131. Ge, N.; Liang, H.; Zhao, Y.Y.; Liu, Y.; Gong, A.J.; Zhang, W.L. Aplysin protects against alcohol-induced liver injury via alleviating oxidative damage and modulating endogenous apoptosis-related genes expression in rats. J. Food. Sci. 2018, 83, $2612-2621$. [CrossRef]

132. He, J.; Liang, H.; Li, Y.; Shi, D.Y.; Ma, A.G. Antioxidant effect of Aplysin on aged mice exposed to D-galatose. Chin. J. Public Health 2009, 25, 1122-1123.

133. Gressler, V.; Stein, É.M.; Dörr, F.; Fujii, M.T.; Colepicolo, P.; Pinto, E. Sesquiterpenes from the essential oil of Laurencia dendroidea (Ceramiales, Rhodophyta): Isolation, biological activities and distribution among seaweeds. Rev. Bras. Farmacogn. 2011, 21, 248-254. [CrossRef]

134. Maneesh, A.; Chakraborty, K. Previously undescribed frido oleanenes and oxygenated labdanes from the brown seaweed Sargassum wightii and their protein tyrosine phosphatase-1B inhibitory activity. Phytochemistry 2017, 144, 19-32. [CrossRef]

135. Alarif, W.M.; Basaif, S.A.; Badria, F.A.; Ayyadd, S.E.N. Two new cytotoxic C-29 steroids from the Red Sea brown alga Cystoseira trinodis. Chem. Nat. Compd. 2015, 51, 697-702. [CrossRef]

136. Choi, J.S.; Han, Y.R.; Byeon, J.S.; Choung, S.Y.; Sohn, H.S.; Jung, H.A. Protective effect of fucosterol isolated from the edible brown algae, Ecklonia stolonifera and Eisenia bicyclis, on tert-butyl hydroperoxide- and tacrine-induced HepG2 cell injury. J. Pharm. Pharmacol. 2015, 67, 1170-1178. [CrossRef]

137. Fernando, I.P.S.; Sanjeewa, K.K.A.; Samarakoon, K.W.; Lee, W.W.; Kim, H.S.; Jeon, Y.J. Squalene isolated from marine macroalgae Caulerpa racemosa and its potent antioxidant and anti-inflammatory activities. J. Food Biochem. 2018, 42, e12628. [CrossRef]

138. Chakraborty, K.; Antony, T. First report of antioxidative abeo-oleanenes from red seaweed Gracilaria salicornia as dual inhibitors of starch digestive enzymes. Med. Chem. Res. 2019, 28, 696-710. [CrossRef]

139. Renju, G.L.; Kurup, G.M.; Kumari, C.H.S. Effect of lycopene from Chlorella marina on high cholesterolinduced oxidative damage and inflammation in rats. Inflammopharmacology 2014, 22, 45-54. [CrossRef]

140. Bai, S.-K.; Lee, S.-J.; Na, H.-J.; Ha, K.-S.; Han, J.-A.; Lee, H.; Kwon, Y.-G.; Chung, C.-K.; Kim, Y.-M. $\beta$-Carotene inhibits inflammatory gene expression in lipopolysaccharide-stimulatedmacrophages by suppressing redox-based NF- $\mathrm{KB}$ activation. Exp. Mol. Med. 2005, 37, 323-334. [CrossRef]

141. de Sousa, C.B.; Gangadhar, K.N.; Macridachis, J.; Pavão, M.; Morais, T.R.; Campino, L.; Varela, J.; Lago, J.H.G. Cystoseira algae (Fucaceae): Update on their chemical entities and biological activities. Tetrahedron Asymmetry 2017, 28, 1486-1505. [CrossRef]

142. Murakami, A.; Nakashima, M.; Koshiba, T.; Maoka, T.; Nishino, H.; Yano, M.; Sumida, T.; Kim, O.K.; Koshimizu, K.; Ohigashi, H. Modifying effects of carotenoids on superoxide and nitric oxide generation from stimulated leukocytes. Cancer Lett. 2000, 149, 115-123. [CrossRef]

143. Camera, E.; Mastrofrancesco, A.; Fabbri, C.; Daubrawa, F.; Picardo, M.; Sies, H.; Stahl, W. Astaxanthin, canthaxanthin and beta-carotene differently affect UVA-induced oxidative damage and expression of oxidative stress-responsive enzymes. Exp. Dermatol. 2009, 18, 222-231. [CrossRef] [PubMed]

144. Fang, Q.; Guo, S.; Zhou, H.; Han, R.; Wu, P.; Han, C. Astaxanthin protects against early burn-wound progression in rats by attenuating oxidative stress-induced inflammation and mitochondria-related apoptosis. Sci. Rep. 2017, 7, 41440. [CrossRef]

145. Niu, T.; Xuan, R.; Jiang, L.; Wu, W.; Zhen, Z.; Song, Y.; Hong, L.; Zheng, K.; Zhang, J.; Xu, Q.; et al. Astaxanthin induces the Nrf2/HO-1 antioxidant pathway in human umbilical vein endothelial cells by generating trace amounts of ROS. J. Agric. Food Chem. 2018, 66, 1551-1559. [CrossRef]

146. Saw, C.L.; Yang, A.Y.; Guo, Y.; Kong, A.N. Astaxanthin and omega-3 fatty acids individually and in combination protect against oxidative stress via the Nrf2-ARE pathway. Food Chem. Toxicol. 2013, 62, 869-875. [CrossRef]

147. Tripathi, D.N.; Jena, G.B. Astaxanthin intervention ameliorates cyclophosphamide-induced oxidative stress, DNA damage and early hepatocarcinogenesis in rat: Role of Nrf2, p53, p38 and phase-II enzymes. Mutat. Res. 2010, 696, 69-80. [CrossRef]

148. Wen, X.; Huang, A.; Hu, J.; Zhong, Z.; Liu, Y.; Li, Z.; Pan, X.; Liu, Z. Neuroprotective effect of astaxanthin against glutamateinduced cytotoxicity in HT22 cells: Involvement of the Akt/GSK-3 $\beta$ pathway. Neuroscience 2015, 303, 558-568. [CrossRef]

149. Wu, Q.; Zhang, X.-S.; Wang, H.-D.; Zhang, X.; Yu, Q.; Li, W.; Zhou, M.-L.; Wang, X.-L. Astaxanthin activates nuclear factor erythroid-related factor 2 and the antioxidant responsive element (Nrf2-ARE) pathway in the brain after subarachnoid hemorrhage in rats and attenuates early brain injury. Mar. Drugs 2014, 12, 6125-6141. [CrossRef] [PubMed]

150. Wu, H.; Niu, H.; Shao, A.; Wu, C.; Dixon, B.J.; Zhang, J.; Yang, S.; Wang, Y. Astaxanthin as a Potential neuroprotective agent for neurological diseases. Mar. Drugs 2015, 13, 5750-5766. [CrossRef]

151. Xue, X.-L.; Han, X.-D.; Li, Y.; Chu, X.-F.; Miao, W.-M.; Zhang, J.-L.; Fan, S.-J. Astaxanthin attenuates total body irradiation-induced hematopoietic system injury in mice via inhibition of oxidative stress and apoptosis. Stem Cell Res. Ther. 2017, 8, 7. [CrossRef] 
152. Yan, T.; Zhao, Y.; Zhang, X.; Lin, X. Astaxanthin inhibits acetaldehyde-induced cytotoxicity in SH-SY5Y cells by modulating Akt/CREB and p38MAPK/ERK signaling pathways. Mar. Drugs 2016, 14, 56. [CrossRef] [PubMed]

153. Taira, J.; Sonamoto, M.; Uehara, M. Dual biological functions of a cytoprotective effect and apoptosis induction by bioavailable marine carotenoid fucoxanthinol through modulation of the nrf2 activation in Raw264.7 macrophage cells. Mar. Drugs 2017, 15, 305. [CrossRef]

154. Heo, S.J.; Ko, S.C.; Kang, S.M.; Kang, H.S.; Kim, J.P.; Kim, S.H.; Lee, K.W.; Cho, M.G.; Jeon, Y.J. Cytoprotective effect of fucoxanthin isolated from brown algae Sargassum siliquastrum against $\mathrm{H}_{2} \mathrm{O}_{2}$-induced cell damage. Eur. Food Res. Technol. 2008, 228, 145-151. [CrossRef]

155. Heo, S.-J.; Jeon, Y.-J. Protective effect of fucoxanthin isolated from Sargassum siliquastrum on UV-B induced cell damage. J. Photochem. Photobiol. B 2009, 95, 101-107. [CrossRef] [PubMed]

156. Kang, M.C.; Lee, S.H.; Lee, W.W.; Kang, N.; Kim, E.A.; Kim, S.Y.; Lee, D.H.; Kim, D.; Jeon, Y.J. Protective effect of fucoxanthin isolated from Ishige okamurae against high-glucose induced oxidative stress in human umbilical vein endothelial cells and zebrafish model. J. Funct. Foods 2014, 11, 304-312. [CrossRef]

157. Ragubeer, N.; Limson, J.L.; Beukes, D.R. Electrochemistry-guided isolation of antioxidant metabolites from Sargassum elegans. Food Chem. 2012, 131, 286-290. [CrossRef]

158. Raguraman, V.; Abraham, L.S.; Mubarak Ali, D.; Narendrakumar, G.; Thirugnanasambandam, R.; Kirubagaran, R.; Thajuddin, N. Unraveling rapid extraction of fucoxanthin from Padina tetrastromatica: Purification, characterization and biomedical application. Process Biochem. 2018, 73, 211-219. [CrossRef]

159. Rajauria, G.; Foley, B.; Abu-Ghannam, N. Characterization of dietary fucoxanthin from Himanthalia elongata brown seaweed. Food Res. Inter. 2017, 99, 995-1001. [CrossRef]

160. Sellimi, S.; Ksouda, G.; Benslima, A.; Nasri, R.; Rinaudo, M.; Nasri, M.; Hajji, M. Enhancing colour and oxidative stabilities of reduced-nitrite turkey meat sausages during refrigerated storage using fucoxanthin purified from the Tunisian seaweed Cystoseira barbata. Food Chem. Toxicol. 2017, 107, 620-629. [CrossRef]

161. Yu, J.; Lin, J.J.; Yu, R.; He, S.; Wang, Q.W.; Cui, W.; Zhang, J.R. Fucoxanthin prevents $\mathrm{H}_{2} \mathrm{O}_{2}$-induced neuronal apoptosis via concurrently activating the PI3-K/Akt cascade and inhibiting the ERK pathway. Food Nutr. Res. 2017, 61, 1304678. [CrossRef]

162. Zhang, Y.; Fang, H.; Xie, Q.; Sun, J.; Liu, R.; Hong, Z.; Yi, R.; Wu, H. Comparative evaluation of the radical-scavenging activities of fucoxanthin and its stereoisomers. Molecules 2014, 19, 2100-2113. [CrossRef]

163. Wang, F.; Huang, L.; Gao, B.; Zhang, C. Optimum production conditions, purification, identification, and antioxidant activity of violaxanthin from microalga Eustigmatos cf. polyphem (Eustigmatophyceae). Mar. Drugs 2018, 16, 190. [CrossRef]

164. Jang, J.H.; Lee, J.H.; Chand, H.S.; Lee, J.S.; Lin, Y.; Weathington, N.; Mallampalli, R.; Jeon, Y.J.; Nyunoya, T. Apo-9'-fucoxanthinone extracted from Undariopsis peteseniana protects oxidative stress-mediated apoptosis in cigarette smoke-exposed human airway epithelial cells. Mar. Drugs 2016, 14, 140. [CrossRef]

165. Kim, H.-S.; Wang, L.; Fernando, I.P.S.; Je, J.-G.; Ko, S.-C.; Kang, M.C.; Lee, J.M.; Yim, M.-J.; Jeon, Y.-J.; Lee, D.-S. Antioxidant efficacy of (-)-loliolide isolated from Sargassum horneri against AAPH-induced oxidative damage in Vero cells and zebrafish models in vivo. J. Appl. Phycol. 2020, 32, 3341-3348. [CrossRef]

166. Jayawardena, T.U.; Kim, H.-S.; Sanjeewa, K.K.A.; Kim, H.-Y.; Rho, J.-R.; Jee, Y.; Ahn, G.; Jeon, Y.-J. Sargassum horneri and isolated 6-hydroxy-4,4,7a-trimethyl-5,6,7,7atetrahydrobenzofuran- 2(4H)-one (HTT); LPS-induced inflammation attenuation via suppressing NF-кB, MAPK and oxidative stress through Nrf2/HO-1 pathways in RAW 264.7 macrophages. Algal Res. 2019, 40, 101513. [CrossRef]

167. de Jesus Raposo, M.F.; de Morais, A.M.M.B.; de Morais, R.M.S.C. Carotenoids from marine microalgae: A valuable natural source for the prevention of chronic diseases. Mar. Drugs 2015, 13, 5128-5155. [CrossRef] [PubMed]

168. Takaichi, S. Carotenoids in algae: Distributions, biosyntheses and functions. Mar. Drugs 2011, 9, 1101-1118. [CrossRef]

169. Kim, H.A.; Miller, A.A.; Drummond, G.R.; Thrift, A.G.; Arumugam, T.V.; Phan, T.G.; Srikanth, V.K.; Sobey, C.G. Vascular cognitive impairment and Alzheimer's disease: Role of cerebral hypoperfusion and oxidative stress. Naunyn Schmiedebergs Arch. Pharmakol. 2012, 385, 953-959. [CrossRef]

170. Padurariu, M.; Ciobica, A.; Lefter, R.; Lacramioara Serban, I.; Stefanescu, C.; Chirita, R. The oxidative stress hypothesis in Alzheimer's disease. Psychiatr. Danub. 2013, 25, 401-409.

171. Fakhri, S.; Aneva, I.Y.; Farzaei, M.H.; Sobarzo-Sánchez, E. The neuroprotective effects of Astaxanthin: Therapeutic targets and clinical perspective. Molecules 2019, 24, 2640. [CrossRef]

172. Wu, H.-J.; Pu, J.-L.; Krafft, P.R.; Zhang, J.-M.; Chen, S. The molecular mechanisms between autophagy and apoptosis: Potential role in central nervous system disorders. Cell. Mol. Neurobiol. 2015, 35, 85-99. [CrossRef]

173. Davinelli, S.; Nielsen, M.E.; Scapagnini, G. Astaxanthin in skin health, repair, and disease: A comprehensive review. Nutrients 2018, 10, 522. [CrossRef]

174. Dembitsky, V.M.; Maoka, T. Allenic and cumulenic lipids. Prog. Lipid Res. 2007, 46, 328-375. [CrossRef] [PubMed]

175. Nomura, T.; Kikuchi, M.; Kubodera, A.; Kawakami, Y. Proton-donative antioxidant activity of fucoxanthin with 1,1-diphenyl-2picrylhydrazyl (DPPH). Biochem. Mol. Biol. Int. 1997, 42, 361-370. [CrossRef]

176. Yan, X.; Chuda, Y.; Suzuki, M.; Nagata, T. Fucoxanthin as the major antioxidant in Hijikia fusiformis, a common edible seaweed. Biosci. Biotechnol. Biochem. 1999, 63, 605-607. [CrossRef] 
177. Fisch, M.K.; Böhm, V.; Wright, A.D.; König, G.M. Antioxidative meroterpenoids from the brown alga Cystoseira crinita. J. Nat. Prod. 2003, 66, 968-975. [CrossRef]

178. De los Reyes, C.; Zbakh, H.; Motilva, V.; Zubía, E. Antioxidant and anti-inflammatory meroterpenoids from the brown alga Cystoseira usneoides. J. Nat. Prod. 2013, 76, 621-629. [CrossRef]

179. Kumagai, M.; Nishikawa, K.; Matsuura, H.; Umezawa, T.; Matsuda, F.; Okino, T. Antioxidants from the brown alga Dictyopteris undulata. Molecules 2018, 23, 1214. [CrossRef] [PubMed]

180. Shimizu, H.; Koyama, T.; Yamada, S.; Lipton, S.A.; Satoh, T. Zonarol, a sesquiterpene from the brown algae Dictyopteris undulata, provides neuroprotection by activating the Nrf2/ARE pathway. Biochem. Biophys. Res. Comm. 2015, 457, 718-722. [CrossRef]

181. Chakraborty, K.; Antony, T.; Joy, M. Prospective natural anti-inflammatory drimanes attenuating pro-inflammatory 5-lipoxygenase from marine macroalga Gracilaria salicornia. Algal Res. 2019, 40, 101472. [CrossRef]

182. Antony, T.; Chakraborty, K. First report of antioxidative $2 H$-chromenyl derivatives from the intertidal red seaweed Gracilaria salicornia as potential antiinflammatory agents. Nat. Prod. Res. 2020, 34, 3470-3482. [CrossRef]

183. Iwashima, M.; Mori, J.; Ting, X.; Matsunaga, T.; Hayashi, K.; Shinoda, D.; Satio, H.; Sankawa, U.; Hayashi, T. Antioxidant and antiviral activities of plastoquinones from the brown alga Sargassum micracanthum, and a new chromene derivative converted from the plastoquinones. Biol. Pharm. Bull. 2005, 28, 374-377. [CrossRef]

184. Mori, J.; Iwashima, M.; Wakasugi, H.; Saito, H.; Matsunaga, T.; Ogasawara, M.; Takahashi, S.; Suzuki, H.; Hayashi, T. New plastoquinones isolated from the brown alga, Sargassum micracanthum. Chem. Pharm. Bull. 2005, 53, 1159-1163. [CrossRef] [PubMed]

185. Gouveia, V.L.M.; Seca, A.M.L.; Barreto, M.C.; Neto, A.I.; Kijjoa, A.; Silva, A.M.S. Cytotoxic meroterpenoids from the macro alga Cystoseira abies-marina. Phytochem. Lett. 2013, 6, 593-597. [CrossRef]

186. De los Reyes, C.; Ortega, M.J.; Zbakh, H.; Motilva, V.; Zubía, E. Cystoseira usneoides: A brown alga rich in antioxidant and antiinflammatory meroditerpenoids. J. Nat. Prod. 2016, 79, 395-405. [CrossRef]

187. Jung, M.; Jang, K.H.; Kim, B.; Lee, B.H.; Choi, B.W.; Oh, K.B.; Shin, J. Meroditerpenoids from the brown alga Sargassum siliquastrum. J. Nat. Prod. 2008, 71, 1714-1719. [CrossRef] [PubMed]

188. Seo, Y.; Lee, H.J.; Park, K.E.; Kim, Y.A.; Ahn, J.W.; Yoo, J.S.; Lee, B.J. Peroxynitrite scavenging constituents from the brown alga Sargassum thunbergii. Biotechnol. Bioprocess Eng. 2004, 9, 212-216. [CrossRef]

189. Seo, Y.; Park, K.E.; Nam, T.J. Isolation of a new chromene from the brown alga Sargassum thunbergii. Bull. Korean Chem. Soc. 2007, 28, 1831-1833.

190. Ham, Y.M.; Kim, K.N.; Lee, W.J.; Lee, N.H.; Hyun, C.G. Chemical constituents from Sargassum micracanthum and antioxidant activity. Int. J. Pharmacol. 2010, 6, 147-151. [CrossRef]

191. Jang, K.H.; Lee, B.H.; Choi, B.W.; Lee, H.S.; Shin, J. Chromenes from the brown alga Sargassum siliquastrum. J. Nat. Prod. 2005, 68, 716-723. [CrossRef] [PubMed]

192. Lee, J.I.; Seo, Y. Chromanols from Sargassum siliquastrum and their antioxidant activity in HT 1080 cells. Chem. Pharm. Bull. 2011, 59, 757-761. [CrossRef]

193. Yoon, W.J.; Kim, K.N.; Heo, S.J.; Han, S.C.; Kim, J.; Ko, Y.J.; Kang, H.K.; Yoo, E.S. Sargachromanol G inhibits osteoclastogenesis by suppressing the activation NF-kappa B and MAPKs in RANKL-induced RAW 264.7 cells. Biochem. Biophys. Res. Commun. 2013, 434, 892-897. [CrossRef]

194. Mori, J.; Iwashima, A.; Takeuchi, M.; Saito, H. A synthetic study on antiviral and antioxidative chromene derivative. Chem. Pharm. Bull. 2006, 54, 391-396. [CrossRef] [PubMed]

195. Seo, Y.; Park, K.E.; Kim, Y.A.; Lee, H.J.; Yoo, J.S.; Ahn, J.W.; Lee, B.J. Isolation of tetraprenyltoluquinols from the brown alga Sargassum thunbergii. Chem. Pharm. Bull. 2006, 54, 1730-1733. [CrossRef] [PubMed]

196. Gacesa, R.; Lawrence, K.P.; Georgakopoulos, N.D.; Yabe, K.; Dunlap, W.C.; Barlow, D.J.; Wells, G.; Young, A.R.; Long, P.F. The mycosporine-like amino acids porphyra-334 and shinorine are antioxidants and direct antagonists of Keap1-Nrf2 binding. Biochimie 2018, 154, 35-44. [CrossRef]

197. Yoshiki, M.; Tsuge, K.; Tsuruta, Y.; Yoshimura, T.; Koganemaru, K.; Sumi, T.; Matsui, T.; Matsumoto, K. Production of new antioxidant compound from mycosporine-like amino acid, porphyra-334 by heat treatment. Food Chem. 2009, 113, 1127-1132. [CrossRef]

198. Tamura, Y.; Takenaka, S.; Sugiyama, S.; Nakayama, R. Occurrence of anserine as an antioxidative dipeptide in a red alga, Porphyra yezoensis. Biosci. Biotechnol. Biochem. 1998, 62, 561-563. [CrossRef] [PubMed]

199. Yan, P.; Li, G.; Wang, C.; Wu, J.; Sun, Z.; Martin, G.E.; Wang, X.; Reibarkh, M.; Saurí, J.; Gustafson, K.-R. Characterization by empirical and computational methods of dictyospiromide, an intriguing antioxidant alkaloid from the marine alga Dictyota coriacea. Org. Lett. 2019, 21, 7577-7581. [CrossRef]

200. Cermeño, M.; Stack, J.; Tobin, P.R.; O’Keeffe, M.B.; Harnedy, P.A.; Stengel, D.B.; FitzGerald, R.J. Peptide identification from a Porphyra dioica protein hydrolysate with antioxidant, angiotensin converting enzyme and dipeptidyl peptidase IV inhibitory activities. Food Funct. 2019, 10, 3421-3429. [CrossRef] [PubMed]

201. Yabuta, Y.; Fujimura, H.; Kwak, C.S.; Enomoto, T.; Watanabe, F. Antioxidant activity of the phycoerythrobilin compound formed from a dried Korean purple laver (Porphyra sp.) during in vitro digestion. Food Sci. Technol. Res. 2010, 16, 347-351. [CrossRef]

202. Cho, M.L.; Lee, H.-S.; Kang, I.-J.; Won, M.-H.; You, S.G. Antioxidant properties of extract and fractions from Enteromorpha prolifera, a type of green seaweed. Food Chem. 2011, 127, 999-1006. [CrossRef] [PubMed] 
203. Hsu, C.-Y.; Chao, P.-Y.; Hu, S.-P.; Yang, C.-M. The antioxidant and free radical scavenging activities of chlorophylls and pheophytins. Food Nutr. Sci. 2013, 4, 35234. [CrossRef]

204. Kang, Y.-R.; Park, J.; Jung, S.K.; Chang, Y.H. Synthesis, characterization, and functional properties of chlorophylls, pheophytins, and Zn-pheophytins. Food Chem. 2018, 245, 943-950. [CrossRef]

205. Pérez-Gálvez, A.; Viera, I.; Roca, M. Carotenoids and chlorophylls as antioxidants. Antioxidants 2020, 9, 505. [CrossRef] [PubMed]

206. Cahyana, A.H.; Shuto, Y.; Kinoshita, Y. Pyropheophytin a as an antioxidative substance from the marine alga, arame (Eisenia bicyclis). Biosci. Biotechnol. Biochem. 1992, 56, 1533-1535. [CrossRef]

207. Kitano, Y.; Murazumi, K.; Duan, J.; Kurose, K.; Kobayashi, S.; Sugawara, T.; Hirata, T. Effect of dietary porphyran from the red alga, Porphyra yezoensis, on glucose metabolism in diabetic KK-Ay mice. J. Nutr. Sci. Vitaminol. 2012, 58, 14-19. [CrossRef]

208. Tziveleka, L.-A.; Ioannou, E.; Roussis, V. Ulvan, a bioactive marine sulphated polysaccharide as a key constituent of hybrid biomaterials: A review. Carbohydr. Polym. 2019, 218, 355-370. [CrossRef] [PubMed]

209. Wijesekara, I.; Pangestuti, R.; Kim, S.-K. Biological activities and potential health benefits of sulfated polysaccharides derived from marine algae. Carbohydr. Polym. 2011, 84, 14-21. [CrossRef]

210. Andrade, P.B.; Barbosa, M.; Matos, R.P.; Lopes, G.; Vinholes, J.; Mouga, T.; Valentão, P. Valuable compounds in macroalgae extracts. Food Chem. 2013, 138, 1819-1828. [CrossRef] [PubMed]

211. Li, Y.X.; Li, Y.; Lee, S.H.; Qian, Z.J.; Kim, S.K. Inhibitors of oxidation and matrix metalloproteinases, floridoside, and Disofloridoside from marine red alga Laurencia undulata. J. Agric. Food Chem. 2010, 58, 578-586. [CrossRef]

212. Wang, B.; Jiang, X.; Jiang, Y.; Hu, X.; Mou, H.; Li, M.; Guan, H. In vitro antioxidative activities of three marine oligosaccharides. Nat. Prod. Res. 2007, 21, 646-654. [CrossRef]

213. Rocha de Souza, M.C.; Marques, C.T.; Dore, C.M.G.; Ferreira da Silva, F.R.; Rocha, H.A.O.; Leite, E.L. Antioxidant activities of sulfated polysaccharides from brown and red seaweeds. J. Appl. Phycol. 2007, 19, 153-160. [CrossRef]

214. Ammar, H.H.; Lajili, S.; Said, R.B.; Le Cerf, D.; Bouraoui, A.; Majdoub, H. Physico-chemical characterization and pharmacological evaluation of sulfated polysaccharides from three species of Mediterranean brown algae of the genus Cystoseira. Daru 2015, 23, 1-8. [CrossRef]

215. Zhao, X.; Xue, C.H.; Li, B.F. Study of antioxidant activities of sulfated polysaccharides from Laminaria japonica. J. Appl. Phycol. 2008, 20, 431-436. [CrossRef]

216. Koh, H.S.A.; Lu, J.; Zhou, W. Structure characterization and antioxidant activity of fucoidan isolated from Undaria pinnatifida grown in New Zealand. Carbohydr. Polym. 2019, 212, 178-185. [CrossRef] [PubMed]

217. Rodriguez-Jasso, R.M.; Mussatto, S.I.; Pastrana, L.; Aguilar, C.N.; Teixeira, J.A. Chemical composition and antioxidant activity of sulphated polysaccharides extracted from Fucus vesiculosus using different hydrothermal processes. Chem. Pap. 2014, 68, 203-209. [CrossRef]

218. Pozharitskaya, O.N.; Obluchinskaya, E.D.; Shikov, A.N. Mechanisms of bioactivities of fucoidan from the brown seaweed Fucus vesiculosus L. of the Barents Sea. Mar. Drugs 2020, 18, 275. [CrossRef]

219. Lim, S.J.; Aida, W.M.W.; Maskat, M.Y.; Mamot, S.; Ropien, J.; Mohd, D.M. Isolation and antioxidant capacity of fucoidan from selected Malaysian seaweeds. Food Hydrocoll. 2014, 42, 280-288. [CrossRef]

220. Kim, K.J.; Yoon, K.Y.; Lee, B.Y. Low molecular weight fucoidan from the sporophyll of Undaria pinnatifida suppresses inflammation by promoting the inhibition of mitogen-activated protein kinases and oxidative stress in RAW264.7 cells. Fitoterapia 2012, 83 , 1628-1635. [CrossRef]

221. Ryu, M.J.; Chung, H.S. Fucoidan reduces oxidative stress by regulating the gene expression of HO1 and SOD1 through the Nrf2/ERK signaling pathway in HaCaT cells. Mol. Med. Rep. 2016, 14, 3255-3260. [CrossRef]

222. Phull, A.R.; Majid, M.; Haq, I.U.; Khan, M.R.; Kim, S.J. In vitro and In vivo evaluation of anti-arthritic, antioxidant efficacy of fucoidan from Undaria pinnatifida (Harvey) Suringar. Int. J. Biol. Macromol. 2017, 97, 468-480. [CrossRef]

223. Zhang, Z.; Zhang, Q.; Wang, J.; Shi, X.; Song, H.; Zhang, J. In vitro antioxidant activities of acetylated, phosphorylated and benzoylated derivatives of porphyran extracted from Porphyra haitanensis. Carbohydr. Polym. 2009, 78, 449-453. [CrossRef]

224. Qi, H.; Zhang, Q.; Zhao, T.; Chen, R.; Zhang, H.; Niu, X.; Li, Z. Antioxidant activity of different sulfate content derivatives of polysaccharide extracted from Ulva pertusa (Chlorophyta) in vitro. Int. J. Biol. Macromol. 2005, 37, 195-199. [CrossRef] [PubMed]

225. Qi, H.; Zhao, T.; Zhang, Q.; Li, Z.; Zhao, Z.; Xing, R. Antioxidant activity of different molecular weight sulfated polysaccharides from Ulva pertusa Kjellm (Chlorophyta). J. Appl. Phycol. 2005, 17, 527-534. [CrossRef]

226. Qi, H.M.; Zhang, Q.B.; Zhao, T.T.; Hu, R.G.; Zhang, K.; Li, Z. In vitro antioxidant activity of acetylated and benzoylated derivatives of polysaccharide extracted from Ulva pertusa (Chlorophyta). Bioorg. Med. Chem. Lett. 2006, 16, 2441-2445. [CrossRef]

227. Rupérez, P.; Ahrazem, O.; Leal, J.A. Potential antioxidant capacity of sulfated polysaccharides from the edible marine brown seaweed Fucus vesiculosus. J. Agric. Food Chem. 2002, 50, 840-845. [CrossRef]

228. Hu, T.; Liu, D.; Chen, Y.; Wu, J.; Wang, S. Antioxidant activity of sulfated polysaccharide fractions extracted from Undaria pinnitafida in vitro. Int. J. Biol. Macromol. 2010, 46, 193-198. [CrossRef]

229. Zhang, Q.B.; Yu, P.Z.; Li, Z.E.; Zhang, H.; Xu, Z.; Li, P.C. Antioxidant activities of sulfated polysaccharide fractions from Porphyra haitanesis. J. Appl. Phycol. 2003, 15, 305-310. [CrossRef]

230. Sun, L.Q.; Wang, L.; Li, J.; Liu, H.H. Characterization and antioxidant activities of degraded polysaccharides from two marine Chrysophyta. Food Chem. 2014, 160, 1-7. [CrossRef] [PubMed] 
231. Phull, A.R.; Kim, S.J. Fucoidan as bio-functional molecule: Insights into the anti-inflammatory potential and associated molecular mechanisms. J. Funct. Foods 2017, 38, 415-426. [CrossRef]

232. Wei, C.-C.; Yen, P.-L.; Chang, S.-T.; Cheng, P.-L.; Lo, Y.-C.; Liao, V.H.-C. Antioxidative activities of both oleic acid and Camellia tenuifolia seed oil are regulated by the transcription factor DAF-16/FOXO in Caenorhabditis elegans. PLoS ONE 2016, 11, e0157195. [CrossRef]

233. Li, Y.X.; Li, Y.; Qian, Z.J.; Kim, M.M.; Kim, S.K. In vitro antioxidant activity of 5-HMF isolated from marine red alga Laurencia undulata in free radical mediated oxidative systems. J. Microbiol. Biotechnol. 2009, 19, 1319-1327. [CrossRef]

234. Makkar, F.; Chakraborty, K. Antioxidant and anti-inflammatory oxygenated meroterpenoids from the thalli of red seaweed Kappaphycus alvarezii. Med. Chem. Res. 2018, 27, 2016-2026. [CrossRef]

235. Chakraborty, K.; Raola, V.K. In vitro bioactive analysis and antioxidant activity of two species of seaweeds from the Gulf of Mannar. Nat. Prod. Res. 2018, 32, 2729-2734. [CrossRef] [PubMed]

236. Maneesh, A.; Chakraborty, K. Previously undescribed antioxidative $O$-heterocyclic angiotensin converting enzyme inhibitors from the intertidal seaweed Sargassum wightii as potential antihypertensives. Food Res. Inter. 2018, 113, 474-486. [CrossRef] [PubMed]

237. Makkar, F.; Chakraborty, K. Highly oxygenated antioxidative $2 H$-chromen derivative from the red seaweed Gracilaria opuntia with pro-inflammatory cyclooxygenase and lipoxygenase inhibitory properties. Nat. Prod. Res. 2018, 32, 2756-2765. [CrossRef]

238. Makkar, F.; Chakraborty, K. Unprecedented antioxidative cyclic ether from the red seaweed Kappaphycus alvarezii with anticyclooxygenase and lipoxidase activities. Nat. Prod. Res. 2017, 31, 1131-1141. [CrossRef]

239. Venkateswarlu, S.; Panchagnula, G.K.; Gottumukkala, A.L.; Subbaraju, G.V. Synthesis, structural revision, and biological activities of $4^{-}$-chloroaurone, a metabolite of marine brown alga Spatoglossum variabile. Tetrahedron 2007, 63, 6909-6914. [CrossRef]

240. Maneesh, A.; Chakraborty, K. Unprecedented antioxidative and anti-inflammatory aryl polyketides from the brown seaweed Sargassum wightii. Food Res. Inter. 2017, 100, 640-649. [CrossRef]

241. Chakraborty, K.; Joseph, D.; Joy, M.; Raola, V.K. Characterization of substituted aryl meroterpenoids from red seaweed Hypnea musciformis as potential antioxidants. Food Chem. 2016, 212, 778-788. [CrossRef] [PubMed]

242. Chakraborty, K.; Dhara, S. First report of substituted $2 \mathrm{H}$-pyranoids from brown seaweed Turbinaria conoides with antioxidant and anti-inflammatory activities. Nat. Prod. Res. 2020, 34, 3451-3461. [CrossRef]

243. Iwasaki, S.; Widjaja-Adhi, M.; Koide, A.; Kaga, T.; Nakano, S.; Beppu, F.; Hosokawa, M.; Miyashita, K. In vivo antioxidant activity of fucoxanthin on obese/diabetes KK-Ay mice. Food Nutr. Sci. 2012, 3, 1491-1499. [CrossRef]

244. Ha, A.W.; Na, S.J.; Kim, W.K. Antioxidant effects of fucoxanthin rich powder in rats fed with high fat diet. Nutr. Res. Pract. 2013, 7, 475-480. [CrossRef] [PubMed] 